UWThPh-2007-01

HWM-06-47

EMPG-06-12

\title{
Localization for Yang-Mills Theory on the Fuzzy Sphere
}

\author{
Harold Steinacker \\ Institut für Theoretische Physik \\ Universität Wien \\ Boltzmanngasse 5, A-1090 Wien, Austria \\ Email: harold.steinacker@univie.ac.at \\ Richard J. Szabo \\ Department of Mathematics \\ and \\ Maxwell Institute for Mathematical Sciences \\ Heriot-Watt University \\ Colin Maclaurin Building, Riccarton, Edinburgh EH14 4AS, U.K. \\ Email: R.J.Szabo@ma.hw.ac.uk
}

\begin{abstract}
We present a new model for Yang-Mills theory on the fuzzy sphere in which the configuration space of gauge fields is given by a coadjoint orbit. In the classical limit it reduces to ordinary Yang-Mills theory on the sphere. We find all classical solutions of the gauge theory and use nonabelian localization techniques to write the partition function entirely as a sum over local contributions from critical points of the action, which are evaluated explicitly. The partition function of ordinary Yang-Mills theory on the sphere is recovered in the classical limit as a sum over instantons. We also apply abelian localization techniques and the geometry of symmetric spaces to derive an explicit combinatorial expression for the partition function, and compare the two approaches. These extend the standard techniques for solving gauge theory on the sphere to the fuzzy case in a rigorous framework.
\end{abstract}




\section{Contents}

1 Introduction and summary $r$

2 Symplectic model for Yang-Mills theory on the fuzzy sphere 3

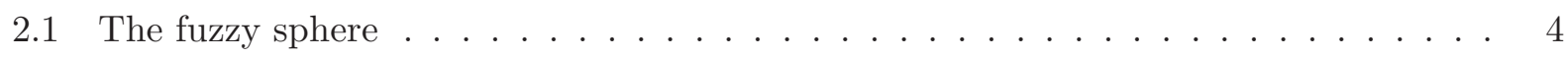

2.2 Configuration space of gauge fields . . . . . . . . . . . . . . . . . . 4

2.3 The Yang-Mills action $\ldots \ldots \ldots \ldots \ldots \ldots$

2.4 Symplectic geometry of the configuration space . . . . . . . . . . . . . 8

3 The classical configuration space $r$

3.1 Classical solutions . . . . . . . . . . . . . . . . . . . . 10

3.2 The classical action . . . . . . . . . . . . . . . . . . . 13

3.3 Local symplectic geometry of the configuration space . . . . . . . . . . . . 13

3.4 Explicit decomposition at Yang-Mills critical surfaces . . . . . . . . . . . . 15

3.5 Fluctuations around the critical surfaces . . . . . . . . . . . . . . 20

4 Nonabelian localization $\quad 21$

4.1 Equivariant cohomology and the localization principle . . . . . . . . . . . . 21

4.2 Explicit evaluation of the localization forms . . . . . . . . . . . . . . 24

4.3 Localization at the vacuum moduli space . . . . . . . . . . . . . . 26

4.4 Localization at maximally irreducible saddle points . . . . . . . . . . . . . . . . 29

5 Abelianization $\quad 36$

$6 \quad$ Itzykson-Zuber localization on the configuration space $\quad 37$

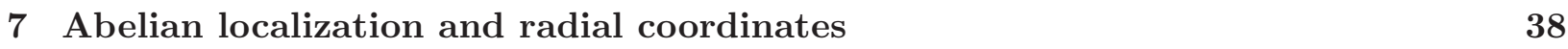

7.1 Polar decomposition of the configuration space . . . . . . . . . . . . . 39

7.2 Evaluation of the abelianized partition function: $U(1)$ gauge theory . . . . . . . . . 40

7.3 Evaluation of the abelianized partition function: $U(n)$ gauge theory $\ldots \ldots . \ldots 4$

8 Yang-Mills critical surfaces in abelianized localization $\quad 45$

8.1 Itzykson-Zuber localization on the symplectic leaves . . . . . . . . . . . . 46

8.2 Radial coordinates for Yang-Mills critical surfaces . . . . . . . . . . . . . . . . 48

8.3 Action of the gauge group $\ldots \ldots \ldots \ldots \ldots \ldots \ldots$ 


\section{Introduction and summary}

Gauge theory on the fuzzy sphere has been of interest for many years as the simplest example of a noncommutative gauge theory with finitely many degrees of freedom which retains all of the classical symmetries of the corresponding undeformed field theory (see for instance [1-12] and references therein). It can be formulated as an $N \times N$ matrix model, which provides a natural regularization preserving all symmetries of quantum gauge theory on the classical sphere which is recovered in the large $N$ limit. At the classical level one finds non-trivial gauge field configurations such as monopoles which can be naturally described in terms of the noncommutative topology of projective modules. Besides Yang-Mills gauge theory which is the focus of this paper, certain other gauge theories on the fuzzy sphere naturally emerge in string theory upon quantizing the worldvolume dynamics of spherical D2-branes [14], obtained for instance as expansions about vacua of matrix models with a Chern-Simons term [15,16] describing superstrings in pp-wave backgrounds [17]. These models contain additional scalar degrees of freedom and are not considered here.

The formulation of Yang-Mills theory as an $N \times N$ matrix model allows a nonperturbative quantization in terms of a finite-dimensional path integral [9]. This can then be evaluated in terms of an $N$-dimensional integral, and the classical result as a sum over two-dimensional instantons [18-20] is recovered in the commutative limit $N \rightarrow \infty$. A different approach to evaluate the path integral was given in [11], which is also restricted to the large $N$ limit. This indicates in particular that the model is void of the usual perturbative ambiguities which plague noncommutative gauge theories in higher dimensions, such as UV/IR mixing (see [21,22] for reviews).

In this paper we will formulate a new model for quantum Yang-Mills theory on the fuzzy sphere, and solve it exactly. The model reduces to pure Yang-Mills theory on the classical sphere when $N \rightarrow$ $\infty$ without any spurious auxilliary scalar fields. The classical theory admits topologically non-trivial solutions as in previous matrix model formulations [9], including some purely noncommutative ones. Its main virtue is that the finite-dimensional configuration space of gauge fields can be described as a compact coadjoint orbit, which is naturally a symplectic manifold with a hamiltonian action of a nonabelian Lie symmetry group. The Yang-Mills action is the square of the corresponding moment map, and therefore our model can be solved exactly using nonabelian localization techniques [18, 23-27] to cast the partition function as a sum over local contributions from the classical solutions of the gauge theory. It can also be solved by abelian localization techniques which exploit the usual Duistermaat-Heckman theorem (see $[28,29]$ for extensive treatments) and which provide an interesting alternative to the semiclassical expansion. Although the model described in this paper is fundamentally different from the fuzzy gauge theories that naturally emerge in string theory, which contain a Chern-Simons term in their action, nonabelian localization bears certain remarkable similarities to the nonabelian localization of Chern-Simons theory on Seifert homology spheres [27].

There are two main motivations behind the present work. Firstly, in the commutative case, twodimensional gauge theories are exactly solvable and can be solved explicitly, either at strong coupling by exploiting the Migdal formula [30,31] which expresses it in terms of a sum over irreducible representations of its gauge group, or at weak coupling by using Poisson resummation techniques to cast it as a sum over two-dimensional instantons [18-20]. One would therefore like to have a similar picture in the noncommutative case. The instanton expansion can be readily generalized to provide the exact solution for gauge theory on a two-dimensional noncommutative torus [32,33]. However, in previous formulations of gauge theory on the fuzzy sphere this is not possible, either because extra scalar degrees of freedom not normally present in commutative Yang-Mills theory destroy the topological nature of the gauge theory and hence its exact solvability, or else because the exact solution does not decompose neatly into isolated contributions from classical solutions. Our model fills this gap, providing a gauge theory on the fuzzy sphere whose exact solution is on a unified footing with that of gauge theory on the noncommutative torus, in the same way that all 
two-dimensional gauge theories admit universal solutions. This is even apparent from the strong coupling expansions of the two noncommutative gauge theories [33,34], which exhibit the same degrees of complexity.

However, the precise implementation of the nonabelian localization principle is rather different in the two cases. In the case of the torus, one starts from a rational noncommutative gauge theory and exploits Morita equivalence with commutative gauge theory to extract the exact instanton expansion, and then uses continuity arguments to extend the expansion to generic values of the noncommutativity parameter. On the fuzzy sphere, Morita equivalence is not available in this manner, and we will have to evaluate the quantum fluctuation integrals required in the semiclassical expansion explicitly. This entails a significantly larger amount of analysis and work than in the case of the torus.

Secondly, our formulation of gauge theory on the fuzzy sphere provides a new finite-dimensional model which can be solved explicitly by nonabelian localization techniques. In particular, we draw heavily on techniques developed recently in [27] to analyse higher critical points in ordinary twodimensional Yang-Mills theory. In our case, the analysis is intrinsically finite-dimensional and in accord with rigorous results established in $[24,26]$. The techniques we exploit in this paper involve a beautiful mix of methods from random matrix theory and (both abelian and nonabelian) localization. In particular, we will throughout compare with some analogous results obtained directly from random matrix theory in [9]. Our approach thereby extends the toolkit of methods which can be generally used to treat gauge theories on fuzzy spaces.

The outline of this paper is as follows. In Section 2 we introduce our new symplectic model for gauge theory on the fuzzy sphere, showing that it reduces to pure Yang-Mills theory on the classical sphere in the large $N$ limit. We also describe in detail the standard construction of the symplectic structure on the coadjoint orbit space of gauge fields. In Section 3 we classify all classical solutions of the gauge theory, finding fuzzy versions of the usual instantons and monopoles as well hosts of purely noncommutative solutions such as fluxons [35]. We then give a detailed description of the local geometry of the configuration space near each Yang-Mills critical point. In Section 4 we review some general aspects of nonabelian localization, and apply it to compute precisely the contributions to the path integral from the vacuum and also higher unstable critical points, showing in each case that the standard instanton contributions on the sphere are recovered at $N \rightarrow \infty$. In Sections 5, 6, and 7 we give an alternative description of the exact path integral in terms of abelian localization, which exploits the fact that the configuration space is a hermitian symmetric space to express the gauge field degrees of freedom in a suitable system of coordinates [36]. These coordinates have been previously used to evaluate integrals arising in random matrix theory in [37,38]. Finally, in Section 8 we compare the abelian and nonabelian localization approaches, indicating how to map between the Yang-Mills critical points and those of the abelianized localization. This is similar to the abelianized localization at higher critical points of ordinary Yang-Mills theory studied in [28], although in the fuzzy case the mapping is not one-to-one and is thus far more intricate.

\section{Symplectic model for Yang-Mills theory on the fuzzy sphere}

In this section we will introduce our new symplectic model for gauge theory on the fuzzy sphere. A similar formulation was given for gauge theory on fuzzy $\mathbb{C} P^{2}$ in [39]. This formulation will be particularly suitable for the approach that we take later on to computing the path integral using localization techniques. 


\subsection{The fuzzy sphere}

Let $N \in \mathbb{N}$, and let $\xi_{i}, i=1,2,3$ be the $N \times N$ hermitian coordinate generators of the fuzzy sphere $S_{N}^{2} \cong \operatorname{Mat}_{N}$ which satisfy the relations

$$
\epsilon^{i j}{ }_{k} \xi_{i} \xi_{j}=\mathrm{i} \xi_{k} \quad \text { and } \quad \xi_{i} \xi^{i}=\frac{1}{4}\left(N^{2}-1\right) \mathbb{1}_{N}
$$

where throughout repeated upper and lower indices are implicitly summed over. The deformation parameter is $\frac{1}{N}$ and $S_{N}^{2}$ becomes the algebra of functions on the classical unit sphere $S^{2}$ in the limit $N \rightarrow \infty$. The quantum space $S_{N}^{2}$ preserves the classical invariance under global rotations as follows. The $\xi_{i}$ generate an $N$-dimensional representation of the global $S U(2)$ isometry group. Under the adjoint action of $S U(2)$, this representation decomposes covariantly into $p$-dimensional irreducible representations $(p)$ of $S U(2)$ as

$$
\operatorname{Mat}_{N} \cong(1) \oplus(3) \oplus \cdots \oplus(2 N-1)
$$

which are interpreted as fuzzy spherical harmonics. This decomposition defines a natural map from $S_{N}^{2}$ to the space of functions on the commutative sphere. The integral of a function $f \in S_{N}^{2}$ over the fuzzy sphere is given by the trace of $f$, which coincides with the usual integral on $S^{2}$

$$
\operatorname{Tr}(f)=\frac{N}{4 \pi} \int_{S^{2}} \mathrm{~d} \Omega f
$$

where the above map is understood. Rotational invariance of the integral then corresponds to invariance of the matrix trace under the adjoint action of $S U(2)$.

Following [9], let us combine the generators $\xi_{i}$ into a larger hermitian $\mathcal{N} \times \mathcal{N}$ matrix

$$
\Xi=\frac{1}{2} \mathbb{1}_{N} \otimes \sigma^{0}+\xi_{i} \otimes \sigma^{i}
$$

where $\mathcal{N}=2 N, \sigma^{0}=\mathbb{1}_{2}$, while

$$
\sigma^{1}=\left(\begin{array}{ll}
0 & 1 \\
1 & 0
\end{array}\right), \quad \sigma^{2}=\left(\begin{array}{cc}
0 & \mathrm{i} \\
-\mathrm{i} & 0
\end{array}\right) \quad \text { and } \quad \sigma^{3}=\left(\begin{array}{cc}
1 & 0 \\
0 & -1
\end{array}\right)
$$

are the Pauli spin matrices obeying

$$
\operatorname{Tr}\left(\sigma^{i}\right)=0 \quad \text { and } \quad \sigma^{i} \sigma^{j}=\delta^{i j} \mathbb{1}_{2}+\mathrm{i} \epsilon^{i j}{ }_{k} \sigma^{k} .
$$

One easily finds from (2.1) and (2.6) the identities

$$
\Xi^{2}=\frac{N^{2}}{4} \mathbb{1}_{\mathcal{N}} \quad \text { and } \quad \operatorname{Tr}(\Xi)=N .
$$

Since $\xi_{i} \otimes \sigma^{i}$ is an intertwiner of the Clebsch-Gordan decomposition $(N) \otimes(2)=(N-1) \oplus(N+1)$, this implies that $\Xi$ has eigenvalues $\pm \frac{N}{2}$ with respective multiplicities $N_{ \pm}=N \pm 1$.

\subsection{Configuration space of gauge fields}

We will now describe the gauge field degrees of freedom in our formulation. To elucidate the construction in as transparent a way as possible, we begin with the abelian case of $U(1)$ gauge theory. To introduce $\mathfrak{u}(N)$ gauge fields $A_{i}$ on $S_{N}^{2}$, consider the covariant coordinates [40]

$$
C_{i}=\xi_{i}+A_{i} \quad \text { and } \quad C_{0}=\frac{1}{2} \mathbb{1}_{N}+A_{0}
$$


which transform under the gauge group $G=U(N)$ as $C_{\mu} \mapsto U^{-1} C_{\mu} U$ for $\mu=0,1,2,3$ and $U \in U(N)$. We can again assemble them into a larger $\mathcal{N} \times \mathcal{N}$ matrix

$$
C=C_{\mu} \otimes \sigma^{\mu} .
$$

Generically, these would consist of four independent fields, and we have to somehow reduce them to two tangential fields on $S_{N}^{2}$. There are several ways to do this. For example, one can impose the constraints $A_{0}=0$ and $C_{i} C^{i}=\frac{N^{2}-1}{4} \mathbb{1}_{\mathcal{N}}$ as in [9], leading to a constrained hermitian multi-matrix model describing quantum gauge theory on the fuzzy sphere which recovers Yang-Mills theory on the classical sphere in the large $N$ limit.

Here we will use a different approach and impose the constraints

$$
C^{2}=\frac{N^{2}}{4} \mathbb{1}_{\mathcal{N}} \quad \text { and } \quad \operatorname{Tr}(C)=N
$$

which is equivalent to requiring that $C$ has eigenvalues $\pm \frac{N}{2}$ with multiplicities $N_{ \pm}=N \pm 1$. In terms of the components of (2.9), this amounts to the constraints

$$
C_{i} C^{i}+C_{0}^{2}=\frac{N^{2}}{4} \mathbb{1}_{\mathcal{N}} \quad \text { and } \quad \mathrm{i} \epsilon_{i}{ }^{j k} C_{j} C_{k}+\left\{C_{0}, C_{i}\right\}=0 .
$$

We checked in Section 2.1 above that this is satisfied for $A_{\mu}=0$, wherein $C=\Xi$. We can then consider the action of the unitary group $U(2 N)$ given by

$$
C \longmapsto U^{-1} C U
$$

which generates a coadjoint orbit of $U(2 N)$ and preserves the constraint (2.10). The gauge fields $A_{\mu}$ are in this way interpreted as fluctuations about the coordinates of the quantum space $S_{N}^{2}$. The constraint (2.10) ensures that the covariant coordinates (2.9) describe a dynamical fuzzy sphere. The gauge group $G=U(N)$ and the global isometry group $S U(2)$ of the sphere are subgroups of the larger symmetry group $U(2 N)$. In particular, the generators of the gauge group are given by elements of the form $\phi=\phi_{0} \otimes \sigma^{0} \in \mathfrak{g}:=\mathfrak{u}(N) \subset \mathfrak{u}(\mathcal{N})$, which defines the gange algebra $\mathfrak{g}$.

We thus claim that a possible configuration space of gauge fields is given by the single coadjoint orbit

$$
\mathcal{O}:=\mathcal{O}(\Xi)=\left\{C=U^{-1} \Xi U \mid U \in U(\mathcal{N})\right\}
$$

where $\Xi \in \mathfrak{u}(2 N)$ is given by (2.4). Explicitly, dividing by the stabilizer of $\Xi$ gives a representation of the orbit (2.13) as the symmetric space $\mathcal{O} \cong U(2 N) / U(N+1) \times U(N-1)$ of dimension $\operatorname{dim}(\mathcal{O})=$ $2\left(N^{2}-1\right)$. A similar construction was given in [39] for the case of $\mathbb{C} P^{2}$, and applied to $S_{N}^{2}$ in a different way in [11]. To justify this claim, we must check that the orbit $\mathcal{O}$ captures the correct number of degrees of freedom at least in the commutative limit $N \rightarrow \infty$, i.e. that the gauge fields $A_{i}$ are essentially tangent vector fields on $S_{N}^{2}$.

The tangent space to $\mathcal{O}(\Xi)$ at a point $C$ is isomorphic to $T_{C} \mathcal{O} \cong \mathfrak{u}(\mathcal{N}) / \mathfrak{r}$, where $\mathfrak{r}=\mathfrak{u}\left(N_{+}\right) \times$ $\mathfrak{u}\left(N_{-}\right)$is the stabilizer subalgebra of $\Xi$. This identification is equivariant with respect to the natural adjoint action of the Lie group $U(\mathcal{N})$. Explicitly, tangent vectors to $\mathcal{O}(\Xi)$ at $C$ have the form ${ }^{1}$

$$
V_{\phi}=\mathrm{i}[C, \phi]
$$

for any hermitian element $\phi \in \mathfrak{u}(\mathcal{N}) / \mathfrak{r},{ }^{2}$ which are just the generators of the unitary group $U(\mathcal{N})$ acting on $\mathcal{O}(\Xi)$ by the adjoint action. These actually describe vector fields on the entire orbit space $\mathcal{O}(\Xi)$. Here and in the following we use the symbol $C$ to denote both elements of $\mathcal{O}(\Xi)$, as well as the matrix of overcomplete coordinate functions on $\mathcal{O}(\Xi)$ defined using the embeddings $\mathcal{O}(\Xi) \hookrightarrow \mathfrak{u}(\mathcal{N}) \hookrightarrow \mathbb{C}^{\mathcal{N}^{2}}$

\footnotetext{
${ }^{1}$ To streamline notation, we will not write explicitly the local dependences of fields and operators defined at points $C \in \mathcal{O}$.

${ }^{2}$ With our conventions, the vector fields (2.14) are real.
} 


\section{The map $\mathcal{J}$}

Following [39], we can make the description of the tangent space to $\mathcal{O}$, spanned by the vectors $V_{\phi}$, more explicit as follows. Consider for $C \in \mathcal{O}$ the map

$$
\mathcal{J}: \mathfrak{u}(\mathcal{N}) \longrightarrow \mathfrak{s u}(\mathcal{N})
$$

defined by

$$
\mathcal{J}(\phi)=\frac{1}{N} V_{\phi}=\frac{\mathrm{i}}{N}[C, \phi] .
$$

Using (2.10) one finds that it satisfies

$$
\mathcal{J}^{3}=-\mathcal{J}
$$

and hence amounts to suitable projectors. Moreover, the map $\mathcal{J}$ is an antihermitian operator with respect to the invariant Cartan-Killing inner product $\operatorname{Tr}(\phi \psi)$ on $\mathfrak{u}(\mathcal{N})$, since

$$
\operatorname{Tr}(\phi \mathcal{J}(\psi))=\frac{\mathrm{i}}{N} \operatorname{Tr}(\phi[C, \psi])=-\frac{\mathrm{i}}{N} \operatorname{Tr}([C, \phi] \psi)=-\operatorname{Tr}(\mathcal{J}(\phi) \psi) .
$$

The map $\mathcal{J}$ will play an instrumental role in this paper and its geometrical properties will be studied in more detail in the next section.

Here we simply note the meaning of $\mathcal{J}$ in the commutative limit $N \rightarrow \infty$. In component form with $\phi=\phi_{\mu} \otimes \sigma^{\mu}$, it acts as ${ }^{3}$

$$
\begin{aligned}
\mathcal{J}(\phi) & \approx-\frac{\mathrm{i}}{N}\left[\phi_{\mu} \otimes \sigma^{\mu}, C_{j} \otimes \sigma^{j}\right] \\
& \approx-\frac{\mathrm{i}}{N}\left[\phi_{\mu}, C_{j}\right] \otimes \sigma^{\mu} \sigma^{j}+\frac{\mathrm{i}}{N} \phi_{\mu} C_{j} \otimes\left[\sigma^{\mu}, \sigma^{j}\right]
\end{aligned}
$$

where we have set $C_{0} \approx \frac{1}{2} \mathbb{1}_{N}$ in the large $N$ limit as will be justified below. Thus at large $N$ this reduces to

$$
\mathcal{J}(\phi) \approx O\left(\frac{1}{N}\right)-\epsilon_{k}^{i j} \phi_{i} x_{j} \otimes \sigma^{k}
$$

for "almost" commutative functions describing the gauge field fluctuations $A_{\mu}$. Here $\xi_{i} \approx \frac{N}{2} x_{i}$ define homogeneous coordinates $x_{i}$ on the sphere. This result means that if we interpret $\phi_{i}$ as a three-component vector field on the fuzzy sphere, including radial components, then the operator $\mathcal{J}$ vanishes on the normal component and essentially coincides with the complex structure for tangential fields on the Kähler manifold $S^{2}$. In particular, the image of $\mathcal{J}$, i.e. the space of tangent vectors $(2.14)$ to $\mathcal{O}(\Xi)$ or small variations of the gauge field, indeed admits two independent field degrees of freedom. This implies that the orbit (2.13) describes two tangent vector fields on $S_{N}^{2}$. Hence the tangent space to $\mathcal{O}$ can be interpreted precisely the space of tangent vector fields on the fuzzy sphere. This nicely reflects the affine nature of the space of gauge fields.

\section{Nonabelian gauge theory}

The generalization to nonabelian $U(n)$ gauge theory is very simple. One now takes

$$
\mathcal{N}=2 n N
$$

and enlarges the matrix (2.4) to $\Xi \otimes \mathbb{1}_{n}$ (which we continue to denote as $\Xi$ for ease of notation). The configuration space is given by the $U(\mathcal{N})$ orbit $(2.13)$ with $C^{2}=\frac{N^{2}}{4} \mathbb{1}_{\mathcal{N}}$ and

$$
\operatorname{Tr}(C)=n N \text {. }
$$

\footnotetext{
${ }^{3}$ Throughout, the notation $\approx$ will always mean an equality which is valid in the large $N$ commutative limit.
} 
Then $C$ has eigenvalues $\pm \frac{N}{2}$ of respective multiplicities $n(N \pm 1)$. The configuration space

$$
\mathcal{O}=U(2 n N) / U\left(n N_{+}\right) \times U\left(n N_{-}\right)
$$

describes $\mathfrak{u}(n)$ - valued gauge fields on $S_{N}^{2}$. Its dimension is given by

$$
\operatorname{dim}(\mathcal{O})=2 n^{2}\left(N^{2}-1\right) .
$$

The gauge group is now given by $G=U(n N)$, and acts on the covariant coordinates $C_{i}=\xi_{i} \otimes$ $\mathbb{1}_{n}+A_{i}, C_{0}=\frac{1}{2} \mathbb{1}_{n N}+A_{0}$ as $C_{\mu} \rightarrow U^{-1} C_{\mu} U$. This leads to the expected transformation law for the $\mathfrak{u}(n)$ - valued gauge fields $A_{i}$. The corresponding gauge algebra is now $\mathfrak{g}:=\mathfrak{u}(n N) \subset \mathfrak{u}(\mathcal{N})$, consisting of elements of the form $\phi=\phi_{0} \otimes \sigma^{0} \in \mathfrak{g}$.

\subsection{The Yang-Mills action}

Consider the action

$$
S=S(C):=\frac{N}{g} \operatorname{Tr}\left(C_{0}-\frac{1}{2} \mathbb{1}_{n N}\right)^{2}
$$

for $C \in \mathcal{O}$, which is invariant under the group of gauge transformations $G$ as well as global $S U(2)$ rotations. We claim that it reduces in the commutative limit $N \rightarrow \infty$ to the usual Yang-Mills action on the sphere $S^{2}$, and can therefore be taken as a definition of the Yang-Mills action on the fuzzy sphere $S_{N}^{2}$. We establish this explicitly below in the abelian case $n=1$, the extension to general $n$ being obvious.

Consider the three-component field strength [9]

$$
\begin{aligned}
F_{i} & :=\mathrm{i} \epsilon_{i}{ }^{j k} C_{j} C_{k}+C_{i} \\
& =\mathrm{i} \epsilon_{i}{ }^{j k}\left[\xi_{j}, A_{k}\right]+\mathrm{i} \epsilon_{i}{ }^{j k} A_{j} A_{k}+A_{i}
\end{aligned}
$$

where $C_{i}=\xi_{i}+A_{i}$ as in (2.8). To understand its significance, consider the "north pole" of $S_{N}^{2}$ where $\xi_{3} \approx \frac{N}{2} x_{3}=\frac{N}{2} \mathbb{1}_{N}$ (with unit radius), and one can replace the operators

$$
\mathrm{i} \mathrm{ad}_{\xi_{i}} \longrightarrow-\varepsilon_{i}^{j} \partial_{j}:=-\varepsilon_{i j} \frac{\partial}{\partial x_{j}}
$$

in the commutative limit for $i, j=1,2$. Hence upon identifying the commutative gauge fields $A_{i}^{\text {cl }}$ through

$$
A_{i}^{\mathrm{cl}}=-\varepsilon_{i}^{j} A_{j}
$$

the "radial" component $F_{3}$ of the field strength (2.26) reduces in the commutative limit to the standard expression

$$
F_{3} \approx \partial_{1} A_{2}^{\mathrm{cl}}-\partial_{2} A_{1}^{\mathrm{cl}}+\mathrm{i}\left[A_{1}^{\mathrm{cl}}, A_{2}^{\mathrm{cl}}\right] .
$$

The constraint (2.11) now implies

$$
\begin{aligned}
F_{i}+\left\{C_{0}-\frac{1}{2} \mathbb{1}_{N}, C_{i}\right\}=F_{i}+\left\{A_{0}, C_{i}\right\} & =0, \\
\left\{\xi_{i}, A^{i}\right\}+A_{0}+A_{i} A^{i}+A_{0} A_{0} & =0 .
\end{aligned}
$$

Since only configurations with $A_{0}=O\left(\frac{1}{N}\right)$ have finite action (2.25) and $\xi_{3}$ is of order $N$, this implies that $A_{3}, F_{1}$ and $F_{2}$ are of order $\frac{1}{N}$ at the north pole, while $A_{1}$ and $A_{2}$ can be finite of order 1 . In particular, only the radial component $F_{3}$ survives the $N \rightarrow \infty$ limit, with

$$
F_{3}=-\left\{A_{0}, C_{3}\right\} \approx-N A_{0} .
$$

This analysis can be made global by considering the "radial" field strength $F_{r}=x^{i} F_{i}$, which reduces to the usual field strength scalar on $S^{2}$. The action (2.25) thus indeed reduces to the usual Yang-Mills action in the commutative limit with dimensionless gauge coupling $g$, giving

$$
S \approx \frac{1}{N g} \operatorname{Tr}\left(F_{r}\right)^{2} \approx \frac{1}{4 \pi g} \int_{S^{2}} \mathrm{~d} \Omega\left(F_{r}\right)^{2} .
$$




\subsection{Symplectic geometry of the configuration space}

The standard Kirillov-Kostant construction makes the orbit space (2.13) into a symplectic manifold [41]. Given two tangent vector fields $V_{\phi}, V_{\psi}$ as above with $\phi, \psi \in \mathfrak{u}(\mathcal{N})$, the symplectic two-form $\omega \in \Omega^{2}(\mathcal{O})$ is defined locally through its pairing with the bivector $V_{\phi} \wedge V_{\psi}$ as

$$
\left\langle\omega, V_{\phi} \wedge V_{\psi}\right\rangle=\mathrm{i} \operatorname{Tr}(C[\phi, \psi]) .
$$

Using trace manipulations it is easy to see that the kernel of this pairing coincides with the stabilizer algebra $\mathfrak{r}$, and hence it is nondegenerate on $\mathcal{O}(\Xi)$. We will derive below an explicit form of $\omega(2.47)$, which allows to verify directly the well-known fact that $\omega$ is closed,

$$
\mathrm{d} \omega=0 .
$$

Thus $\omega$ indeed defines an invariant symplectic structure on $\mathcal{O}(\Xi)$.

The tangent vectors $V_{\phi}$ are hamiltonian vector fields, and we claim that their generator is given by

$$
H_{\phi}=\operatorname{Tr}(\phi C)
$$

for $\phi \in \mathfrak{u}(\mathcal{N})$. Indeed, then $\mathrm{d} H_{\phi}=\operatorname{Tr}(\phi \mathrm{d} C)$, and by using the dual evaluation

$$
\left\langle\mathrm{d} C, V_{\phi}\right\rangle=\mathrm{i}[C, \phi]
$$

one has

$$
\begin{aligned}
\left\langle\mathrm{d} H_{\phi}, V_{\psi}\right\rangle & =\mathrm{i} \operatorname{Tr}(\phi[C, \psi]) \\
& =-\mathrm{i} \operatorname{Tr}(C[\phi, \psi]) \\
& =-\left\langle\omega, V_{\phi} \wedge V_{\psi}\right\rangle=-\left\langle\iota_{V_{\phi}} \omega, V_{\psi}\right\rangle
\end{aligned}
$$

where $\iota_{V_{\phi}}$ denotes contraction with the vector field $V_{\phi}$. Thus

$$
\mathrm{d} H_{\phi}=-\iota_{V_{\phi}} \omega
$$

as claimed. This means that the hamiltonian function (2.35) defines a periodic flow generated by the action of a one-parameter subgroup $C \mapsto \mathrm{e}^{\mathrm{i} t \phi} C \mathrm{e}^{-\mathrm{i} t \phi}, t \in \mathbb{R}$. The corresponding equivariant moment map $\mu: \mathcal{O}(\Xi) \rightarrow \mathfrak{u}(\mathcal{N})^{\vee}$ is the inclusion map which has the pairings

$$
\langle\mu(C), \phi\rangle=H_{\phi},
$$

and it defines a representation of the Lie algebra $\mathfrak{u}(\mathcal{N})$ through the Poisson algebra corresponding to $\omega$.

For gauge transformations $\phi=\phi_{0} \otimes \sigma^{0}$, the moment map $\mu$ reduces to

$$
\langle\mu(C), \phi\rangle=2 \operatorname{Tr}\left(\phi_{0} C_{0}\right)=\operatorname{Tr}\left(\phi_{0}\left(\mathbb{1}_{n N}+2 A_{0}\right)\right) .
$$

In the commutative limit and for abelian gauge fields $n=1$, this becomes

$$
\langle\mu(C), \phi\rangle \approx \operatorname{Tr}\left(\phi_{0}\right)-\frac{2}{N} \operatorname{Tr}\left(\phi_{0} F_{r}\right) \approx-\frac{1}{2 \pi} \int_{S^{2}} \mathrm{~d} \Omega \phi_{0} F_{r}
$$

up to an irrelevant shift, which is just the anticipated moment map for Yang-Mills theory on the classical sphere [18]. Given the appropriate symplectic structure and moment map on the gauge field configuration space $\mathcal{O}$, the nonabelian localization principle for two-dimensional Yang-Mills theory can be applied for the action constructed as the square of the moment map. This is precisely the Yang-Mills action on $S_{N}^{2}$ given in (2.25). The constant term $\frac{1}{2} \mathbb{1}_{n N}$ is just the first Chern number of a background gauge field configuration and is of no significance for this discussion. This procedure will be worked out in detail in Section 4 . 
More about the symplectic form

For later use, we will now derive some properties of the symplectic form introduced in (2.33). Consider the i $\mathfrak{u}(\mathcal{N})$-valued one-form on $\mathcal{O}(\Xi)$ given by

$$
\theta:=C^{-1} \mathrm{~d} C .
$$

Given the constraints (2.10) and using $\mathrm{d} C^{2}=0$, this can be rewritten as

$$
\theta=\frac{4}{N^{2}} C \mathrm{~d} C=\frac{2}{N^{2}}[C, \mathrm{~d} C] .
$$

It obeys the constraints

$$
\mathrm{d} \theta+\theta^{2}=0 \quad \text { and } \quad \operatorname{Tr}(\theta)=0 .
$$

Thus $\theta \in \Omega^{1}(\mathcal{O}, \mathrm{i} \mathfrak{u}(\mathcal{N}))$ is essentially the canonical invariant Maurer-Cartan one-form, with the additional property

$$
[C, \theta]=-2 \mathcal{J}^{2}(\mathrm{~d} C)=2 \mathrm{~d} C
$$

where we have used the fact that $\mathrm{d} C$ is tangent to the orbit space and applied the projection property (2.17). In particular, along with the fact that $C^{2}$ is constant, this implies that

$$
C \theta+\theta C=0 .
$$

Using again the constraint (2.10), the symplectic two-form (2.33) can be written as

$$
\omega=-\frac{\mathrm{i}}{2 N^{2}} \operatorname{Tr}(C[\mathrm{~d} C, \mathrm{~d} C])=\frac{\mathrm{i}}{4} \operatorname{Tr}\left(C \theta^{2}\right) .
$$

To see this, we substitute this expression using (2.18) and (2.17) into

$$
\begin{aligned}
\left\langle\omega, V_{\phi} \wedge V_{\psi}\right\rangle & =-\frac{\mathrm{i}}{N^{2}} \operatorname{Tr}(C[[C, \phi],[C, \psi]]) \\
& =\mathrm{i} \operatorname{Tr}(C[\mathcal{J}(\phi), \mathcal{J}(\psi)]) \\
& =\mathrm{i} \operatorname{Tr}([C, \mathcal{J}(\phi)] \mathcal{J}(\psi)) \\
& =-N \operatorname{Tr}\left(\mathcal{J}^{3}(\phi) \psi\right) \\
& =N \operatorname{Tr}(\mathcal{J}(\phi) \psi) \\
& =\mathrm{i} \operatorname{Tr}([C, \phi] \psi)=\mathrm{i} \operatorname{Tr}(C[\phi, \psi])
\end{aligned}
$$

for any $\phi, \psi \in \mathfrak{u}(\mathcal{N})$, which coincides with the definition (2.33). Using (2.45) and (2.46), this identity gives a simple proof of the closure property (2.34) as

$$
\mathrm{d} \omega=\frac{\mathrm{i}}{4} \operatorname{Tr}\left(\mathrm{d} C \theta^{2}\right)=-\frac{\mathrm{i}}{8} \operatorname{Tr}\left([\theta, C] \theta^{2}\right)=0 .
$$

\section{The classical configuration space}

In this section we will investigate in detail the space of classical solutions of $U(n)$ gauge theory on the fuzzy sphere $S_{N}^{2}$ defined by the action (2.25). Understanding this space will be crucial for the exact solution of the quantum gauge theory, which as we will see in the next section is given exactly by its semiclassical expansion. We will first classify the solutions to the classical equations of motion, over which the partition function will be summed. Among these solutions we will find a variety of fluxons and, as in the case of gauge theory on the noncommutative torus, only a very small subset of all two-dimensional noncommutative instantons on $S_{N}^{2}$ map into the usual instantons of Yang-Mills theory on $S^{2}$ in the commutative limit $N \rightarrow \infty$. We will then thoroughly describe the local symplectic geometry of the configuration space $\mathcal{O}$ near each critical point of the Yang-Mills action, as symplectic integrals over these neighbourhoods will produce the required quantum fluctuation determinants in the semiclassical expansion. 


\subsection{Classical solutions}

The critical points of the Yang-Mills action (2.25) are easy to find. Since the most general variation of a gauge field $C \in \mathcal{O}$ is given by $\delta C=[C, \phi]$, by varying $(2.25)$ one finds that the critical points satisfy

$$
0=\operatorname{Tr}\left(\delta C_{0}\left(C_{0}-\frac{1}{2} \mathbb{1}_{n N}\right)\right)=\operatorname{Tr}\left([C, \phi] C_{0}\right)=\operatorname{Tr}\left(\phi\left[C_{0}, C\right]\right)
$$

for arbitrary $\phi \in \mathfrak{u}(\mathcal{N}) / \mathfrak{r}$. They are therefore given by solutions of the equation $\left[C_{0}, C\right]=0$, which agrees with the known saddle-points in the matrix model formulation of [9]. This equation is equivalent to

$$
\left[C_{0}, C_{i}\right]=0
$$

which together with (2.11) implies that

$$
\begin{aligned}
{\left[C_{i}, C_{j}\right] } & =\mathrm{i} \epsilon_{i j}{ }^{k}\left(2 C_{0}\right) C_{k}, \\
C_{0}^{2} & =\frac{N^{2}}{4} \mathbb{1}_{n N}-C_{i} C^{i} .
\end{aligned}
$$

For solutions with $C_{0} \neq 0$, we can use (3.2) to define

$$
L_{i}=\frac{1}{2 C_{0}} C_{i}
$$

and rewrite (3.3) as

$$
\begin{aligned}
{\left[L_{i}, L_{j}\right] } & =\mathrm{i} \epsilon_{i j}{ }^{k} L_{k}, \\
L_{i} L^{i} & =\left(\frac{N^{2}}{4 C_{0}^{2}}-\frac{1}{4}\right) \mathbb{1}_{n N} .
\end{aligned}
$$

These equations mean that the critical points of the Yang-Mills action correspond to (isomorphism classes of $)(n N) \times(n N)$ unitary representations of the isometry group $S U(2)$, i.e. homomorphisms $\pi_{n N}: S U(2) \rightarrow U(n N)$. Up to isomorphism, for each integer $p \geq 1$ there is a unique irreducible $S U(2)$ representation $(p)$ of dimension $p$. Therefore, there is a one-to-one correspondence between classical solutions and ordered partitions $\left(n_{1}, \ldots, n_{k}\right)$ of the integer $n N=n_{1}+\cdots+n_{k}$, with $n_{i}$ the dimension of the $i$-th irreducible subrepresentation in the representation $\pi_{n N}$ characterizing the given critical point. Each such classical solution breaks the $U(n N)$ gauge symmetry locally to the centralizer $\prod_{i} U\left(k_{i}\right)$ of the homomorphism $\pi_{n N}$, where $k_{i}$ denotes the multiplicity of the blocks. They can be seen [9] to give precisely the usual two-dimensional instantons for $U(n)$ Yang-Mills theory on $S^{2}$. These solutions also agree with those that can be interpreted as configurations of D0-branes inside D2-branes [14], although the ones which will survive the large $N$ limit are different.

Therefore, each critical point is labelled (up to gauge equivalence) by the set of dimensions $n_{i}$ of the irreducible representations, supplemented with a sign $s_{i}$ which is defined by $s_{i}=\operatorname{sgn}\left(C_{0}\left(n_{i}\right)\right)=$ \pm 1 (in that representation) when $C_{0}\left(n_{i}\right) \neq 0$ and $s_{i}=0$ if $C_{0}\left(n_{i}\right)=0$. We can thereby label the critical surfaces, i.e. the connected components of the moduli space of classical solutions in $\mathcal{O}$, as

$$
\mathcal{C}_{\left(n_{1}, s_{1}\right), \ldots,\left(n_{k}, s_{k}\right)} \quad \text { with } \quad n_{i} \in \mathbb{N} \quad \text { and } \quad s_{i} \in\{ \pm 1,0\}
$$

with the constraints

$$
1 \leq n_{1} \leq n_{2} \leq \cdots \leq n_{k}, \quad \sum_{i=1}^{k} n_{i}=n N \quad \text { and } \quad \sum_{i=1}^{k} s_{i}=n
$$


and $s_{i}=0$ only if $n_{i}=1$. Any non-trivial irreducible representation with $n_{i}>1$ and $C_{0} \neq 0$ gives a contribution $\pm N$ to the trace $\operatorname{Tr}(C)$, which must be balanced in order to satisfy the eigenvalue multiplicity constraint (2.22). This is the role of the condition $\sum_{i} s_{i}=n$ in (3.7). Note that one can change the sign of any individual irreducible representation.

The meaning of the blocks $\left(n_{i}, s_{i}\right)$ can be described as follows:

- $\underline{s_{a}= \pm 1:}$ In this case $C_{0} \neq 0$, and hence $\left\|C_{0}\right\|>\frac{1}{2}$ due to (3.5). These solutions come with two signs. Note that any irreducible representation with small dimension will be highly suppressed in the large $N$ limit. The most extreme case is a sum of trivial representations, with $n_{a}=1$, for which

$$
C_{i}=0 \quad \text { and } \quad C_{0}\left(n_{a}=1\right)=s_{a} \frac{N}{2}
$$

- $\underline{s_{a}=0:}$ In this case $C_{0}=0$ and $n_{a}=1$, which implies that $C_{i}=c_{i}$ with $c_{i} \in \mathbb{R}$ and $\frac{N^{2}}{4}=c_{i} c^{i}$. These solutions are also suppressed at large $N$ but less so than those with $C_{i}=0$ above. They correspond to fluxons [35] whose positions on $S^{2}$ are determined by the vector $c_{i}$.

Note that each such saddle-point (or more generally any gauge field configuration $C$ ) defines a projective module over the fuzzy sphere algebra $S_{N}^{2}$, obtained by writing $C$ in $2 n \times 2 n$ block-matrix form. The module then corresponds to a projector $\Pi_{\left(n_{1}, s_{1}\right), \ldots,\left(n_{k}, s_{k}\right)} \in \operatorname{Mat}_{2 n}\left(S_{N}^{2}\right)$. Let us describe some of these critical points explicitly.

\section{Ground state}

The vacuum solution has $k=n$ and is given by the critical surface $\mathcal{C}_{(N, 1), \ldots,(N, 1)}$, which implies that $C_{0}=\frac{1}{2} \mathbb{1}_{n N}$. It follows that $C_{i} C^{i}=\frac{N^{2}-1}{4} \mathbb{1}_{N}$, which is the quadratic Casimir invariant of the $N$-dimensional irreducible representation of $S U(2)$. Using a suitable $U(n N)$ gauge transformation, it can be written as

$$
C_{i}=\xi_{i} \otimes \mathbb{1}_{n}
$$

and we recover the original coordinates of the fuzzy sphere $S_{N}^{2}$. This is equivalent to the vanishing curvature condition $F=0$. In the abelian case $n=1$, an application of Schur's lemma shows that the only matrix which commutes with $C$ is the constant matrix and so the gauge group $U(N)$ acts freely on the moduli space of vacuum solutions, corresponding simply to a change of basis in this case. For $n>1$ the solution is a direct sum of $n$ identical representations. This commutes with the action of $\mathfrak{u}(n)$, and so now the gauge group $U(n N)$ contains a non-trivial stabilizer. The moduli space of flat connections is therefore isomorphic to the smooth manifold $U(n N) / U(n)$ in the nonabelian case. Note that any configuration near the vacuum, with small but finite action, is given by a small deformation of an irreducible $S U(2)$ representation describing $S_{N}^{2}$, and in particular the gauge field fluctuations $A_{\mu}$ are "small". It is in this sense that the quantum gauge theory will describe a fluctuating theory of noncommutative fuzzy sphere geometries.

\section{Fluxons}

At the other extreme, if $C_{0}$ has several zero eigenvalues, i.e. several fluxons, the situation is much more complicated. For example, when $C_{0}=0$ and $n=1$ we obtain a fuzzy version of the moduli space of constant curvature connections in genus 0 provided by the critical surface

$$
\mu^{-1}\left(C_{0}=0\right)=\left\{C_{i} \in \mathfrak{u}(N) \mid C_{i} C^{i}=\frac{N^{2}}{4} \mathbb{1}_{N},\left[C_{i}, C_{j}\right]=0\right\}
$$


along with the condition (2.22) on the multiplicities of the eigenvalues of $C_{i} \otimes \sigma^{i}$. The action of the $U(N)$ gauge group on (3.10) can be used to simultaneously diagonalize the three matrices $C_{i}$. The Marsden-Weinstein symplectic reduction of the orbit space $\mathcal{O}(\Xi)$ is then essentially a symmetric product orbifold of the classical sphere $S^{2}$ given by

$$
\mathcal{M}_{0}:=\mu^{-1}\left(C_{0}=0\right) / / U(N) \cong \operatorname{Sym}^{N}\left(S^{2}\right),
$$

where $\operatorname{Sym}^{N}\left(S^{2}\right):=\left(S^{2}\right)^{N} / \mathfrak{S}_{N}$ and the quotient by the Weyl group $\mathfrak{S}_{N} \subset U(N)$ is the residual gauge symmetry acting by permutations of the real eigenvalues of the hermitian matrices $C_{i}$ representing the positions of the fluxons on $S^{2}$, which are indistinguishable. The fluxon moduli space $\mathcal{M}_{0}$ contains orbifold singularities arising from the fixed points of the $\mathfrak{S}_{N}$-action on $\left(S^{2}\right)^{N}$, which occur whenever two or more fluxon locations coincide. This is analogous to the vacuum solution of two-dimensional $U(N)$ gauge theory on a noncommutative torus wherein the moduli space of constant curvature connections is the symmetric product orbifold $\operatorname{Sym}^{N}\left(T^{2}\right)$ [32], and there is a natural correspondence between two-dimensional noncommutative instantons and fluxons [42]. In the present case the $U(N)$ action on the fluxon configuration space (3.10) also has additional fixed points. Note that the restriction of the symplectic two-form (2.47) to the moduli space $\mathcal{M}_{0}$ is given by

$$
\left.\omega\right|_{\mathcal{M}_{0}}=-\frac{4 \mathrm{i}}{N^{2}} \sum_{a=1}^{N} \epsilon^{i j k} c_{i}^{a} \mathrm{~d} c_{j}^{a} \wedge \mathrm{d} c_{k}^{a}
$$

where $c_{i}^{a} \in \mathbb{R}$ are the eigenvalues of $C_{i}$ with $\sum_{i}\left(c_{i}^{a}\right)^{2}=\frac{N^{2}}{4}$ for each $a=1, \ldots, N$. With the usual embedding of the two-sphere $S^{2} \hookrightarrow \mathbb{R}^{3}$, this is just the standard round symplectic two-form on the Kähler manifold $\left(S^{2}\right)^{N}$. Each fluxon contributes a suppression factor $\mathrm{e}^{-\frac{N}{4 g}}$ due to $(2.25)$.

\section{Instantons on $S^{2}$}

The configurations which will dominate the path integral in the large $N$ classical limit are the low-energy solutions with small actions. These are solutions with $n$ partitions and critical surfaces $\mathcal{C}_{\left(n_{1}, 1\right), \ldots,\left(n_{n}, 1\right)}$ with $n_{i} \approx N$. They correspond to the usual instantons of $U(n)$ gauge theory on $S^{2}$ with vanishing $U(1)$ flux, as shown in [9]. These solutions may also contain additional fluxons, which behave like localized flux tubes which ensure that the total $U(1)$ flux vanishes. Their contributions are suppressed by factors of at least $\mathrm{e}^{-\frac{N}{4 g}}$, however they do contribute in the double scaling, quantum plane limit wherein $S_{N}^{2}$ becomes noncommutative $\mathbb{R}^{2}[43,44]$.

\section{Monopoles}

As shown in $[9,13]$, an irreducible representation with $n_{i}=N-m_{i}$ corresponds to the gauge field of a monopole with magnetic charge $m_{i} \in \mathbb{Z}$. Configurations with non-trivial $U(1)$ monopole number can therefore be obtained by relaxing the constraint (2.22) and replacing it by

$$
\operatorname{Tr}(C)=n N-\mathrm{c}_{1}
$$

where $c_{1}=\sum_{i} m_{i} \in \mathbb{Z}$ is the first Chern number. In order to maintain the constraint $C^{2}=\frac{N^{2}}{4} \mathbb{1}_{\mathcal{N}}$, the matrix dimension (2.21) must then be replaced with $\mathcal{N}=2\left(n N-c_{1}\right)$.

Some of these nontrivial $U(1)$ bundles are realized within the original configuration space (2.23), in the presence of trivial blocks with $n_{a}=1, s_{a}= \pm 1$. For example, in the abelian case $n=1$ the solutions in $\mathcal{C}_{(N-2,1),(1,1),(1,-1)}$ are naturally interpreted as monopoles with charge $m=2$. The blocks $(1, \pm 1)$ have vanishing field strength $F_{i}=0$, and are naturally interpreted as Dirac strings. They are suppressed by factors of at least $\mathrm{e}^{-N^{3} / g}$. Replacing the trivial blocks with fluxons leads to vanishing global $U(1)$ flux as discussed above. 


\subsection{The classical action}

The values of the Yang-Mills action (2.25) on the classical solutions obtained in Section 3.1 above will determine the classical contributions to the path integral in the next section. The action at these critical points can be evaluated as follows. Note that for each $p$-dimensional irreducible representation $L_{i}$ of the isometry group $S U(2)$, one has $L_{i} L^{i}=\frac{p^{2}-1}{4} \mathbb{1}_{p}$ and hence from (3.5) it follows that

$$
\frac{N^{2}}{p^{2}} \mathbb{1}_{p}=4 C_{0}(p)^{2}
$$

on that representation, so that $C_{0}(p)= \pm \frac{N}{2 p} \mathbb{1}_{p}$. Consider the reduced Yang-Mills action

$$
S^{\prime}:=\frac{N}{g} \operatorname{Tr}\left(C_{0}^{2}\right)=S+\frac{N}{g} \operatorname{Tr}\left(C_{0}\right)-\frac{N}{4 g} \operatorname{Tr}\left(\mathbb{1}_{n N}\right)=S+\frac{n N^{2}}{4 g}
$$

which is somewhat easier to manipulate than $S$. For a dominant solution with critical surface $\mathcal{C}_{\left(n_{1}, 1\right), \ldots,\left(n_{n}, 1\right)}$ and $n_{i}>1$, the action $S^{\prime}$ is given by

$$
S^{\prime}\left(\left(n_{1}, 1\right), \ldots,\left(n_{n}, 1\right)\right)=\frac{N}{g} \sum_{i=1}^{n} n_{i} \frac{N^{2}}{4 n_{i}^{2}}=\frac{N^{3}}{4 g} \sum_{i=1}^{n} \frac{1}{n_{i}} .
$$

While possible fluxon blocks with $n_{i}=1$ do not contribute at all to $S^{\prime}$, they do contribute $\frac{N}{4 g}$ to the original action $S(2.25)$. Their total contributions to $S$ is proportional to the fluxon charge, i.e. the total number of blocks with $n_{i}=1$, and agrees with the usual fluxon action [35] in the quantum plane limit of $S_{N}^{2}$ [43].

The dominant configurations in the classical limit are therefore those with

$$
n_{i}=N-m_{i} \quad \text { and } \quad \sum_{i=1}^{n} m_{i}=0
$$

with small $m_{i} \in \mathbb{Z}$, for which

$$
C_{0}\left(n_{i}\right)=\frac{N}{2\left(N-m_{i}\right)} \mathbb{1}_{n_{i}} \approx \frac{1}{2}\left(1+\frac{m_{i}}{N}\right) \mathbb{1}_{n_{i}} .
$$

Note that then

$$
\operatorname{Tr}\left(C_{0}\right)=\sum_{i=1}^{n}\left(N-m_{i}\right) \frac{N}{2\left(N-m_{i}\right)}=\frac{n N}{2}
$$

as required. It follows that

$$
S\left(\left(n_{1}, 1\right), \ldots,\left(n_{n}, 1\right)\right) \approx \frac{N}{g} \sum_{i=1}^{n}\left(N-m_{i}\right)\left(\frac{m_{i}}{2 N}\right)^{2}+O\left(\frac{1}{N}\right) \approx \frac{1}{4 g} \sum_{i=1}^{n} m_{i}^{2},
$$

which is the usual expression $[19,20]$ for the classical action of $U(n)$ Yang-Mills theory on the sphere $S^{2}$ with trivial gauge bundle evaluated on the two-dimensional instanton on $S^{2}$ corresponding to a configuration of $n$ Dirac monopoles of magnetic charges $m_{i} \in \mathbb{Z}$. Non-trivial gauge bundles over $S^{2}$ of first Chern class $c_{1} \in \mathbb{Z}$ are obtained by modifying the trace constraint as in (3.13).

\subsection{Local symplectic geometry of the configuration space}

We will now develop the local symplectic geometry of the configuration space of gauge fields near each Yang-Mills critical point. This is done by analysing in more detail the map (2.16), satisfying (2.17). We want to find a useful description of the tangent space $T_{C} \mathcal{O} \cong \operatorname{im}(\mathcal{J})$, i.e. of the 
local geometry of the orbit space $\mathcal{O}$. Since $\mathcal{J}$ is an anti-hermitian operator with respect to the Cartan-Killing form on $\mathfrak{u}(\mathcal{N})$ (see $(2.18)$ ), it follows that the space $\mathfrak{u}(\mathcal{N})$ splits into two orthogonal subspaces as

$$
\mathfrak{u}(\mathcal{N})=\operatorname{ker}(\mathcal{J}) \oplus \operatorname{ker}\left(\mathcal{J}^{2}+\mathbb{1}_{\mathcal{N}}\right)
$$

where $\operatorname{ker}(\mathcal{J})=\mathfrak{r}=\mathfrak{u}\left(n N_{+}\right) \oplus \mathfrak{u}\left(n N_{-}\right)$is the stabilizer subalgebra, while $\operatorname{ker}\left(\mathcal{J}^{2}+\mathbb{1}_{\mathcal{N}}\right) \cong T_{C} \mathcal{O}$ is the tangent space to the configuration space at $C \in \mathcal{O}$. In particular, $\mathcal{J}$ defines a complex structure on $T_{C} \mathcal{O}$, and (3.21) is just the Cartan decomposition of $\mathfrak{u}(\mathcal{N})$ corresponding to the symmetric space $\mathcal{O}$. This follows immediately by noticing that the involutive automorphism

$$
\mathrm{j}: \mathfrak{u}(\mathcal{N}) \longrightarrow \mathfrak{u}(\mathcal{N}), \quad \phi \longmapsto C \phi C^{-1}
$$

is $\mathbb{1}_{\mathcal{N}}$ on $\operatorname{ker}(\mathcal{J})$ and $-\mathbb{1}_{\mathcal{N}}$ on $\operatorname{ker}\left(\mathcal{J}^{2}+\mathbb{1}_{\mathcal{N}}\right)$ upon using the constraints (2.10). Moreover, for any $V_{\phi}, V_{\psi} \in T_{C} \mathcal{O}$, from (2.47) one has

$$
\left\langle\omega, V_{\phi} \wedge V_{\psi}\right\rangle=\frac{\mathrm{i}}{N^{2}} \operatorname{Tr}\left(\left[C, V_{\phi}\right] V_{\psi}\right)=\frac{1}{N} \operatorname{Tr}\left(\mathcal{J}\left(V_{\phi}\right) V_{\psi}\right)
$$

and

$$
\left\langle\omega, V_{\phi} \wedge \mathcal{J}\left(V_{\psi}\right)\right\rangle=\frac{1}{N} \operatorname{Tr}\left(V_{\phi} V_{\psi}\right),
$$

expressing the fact that the symplectic two-form $\omega$ makes the configuration space $\mathcal{O}$ into a Kähler manifold with respect to the complex structure (2.16). All of these properties are just standard features of hermitian symmetric spaces [36], as will be exploited at length in this paper.

Consider the restriction of the map $\mathcal{J}$ to the gauge algebra $\mathfrak{g}=\mathfrak{u}(n N) \subset \mathfrak{u}(\mathcal{N})$ containing elements of the form $g=\phi \otimes \sigma^{0}$. Since $\mathcal{J}(\phi)$ is the infinitesimal gauge transformation of the gauge field $C$ generated by $\phi$, it describes the orbits of the gauge group $G=U(n N)$ acting on the configuration space $\mathcal{O}$, in $T_{C} \mathcal{O}$. Generically this action is free (apart from the trivial $\mathfrak{u}(1)$ ), but not for certain critical points. For example, for the vacuum solution (3.9) the subalgebra $\mathbb{1}_{N} \otimes \mathfrak{u}(n)$ commutes with $C$. The higher critical points in the nonabelian case generically have a smaller $\mathfrak{u}(1)^{n}$ centralizer algebra.

More precisely, consider the kernel of $\mathcal{J}$ at $C$ restricted to the gauge algebra $\mathfrak{g}$,

$$
\mathfrak{s}:=\operatorname{ker}(\mathcal{J}) \cap \mathfrak{g},
$$

which is the subgroup of the gauge group that stabilizes $C$. The elements $\phi \in \mathfrak{s}$ are orthogonal to $T_{C} \mathcal{O}$ due to (3.21). Hence $\mathfrak{g}$ decomposes into orthogonal subspaces

$$
\mathfrak{g}=\mathfrak{s} \oplus \mathfrak{g}^{\prime}
$$

where $\mathfrak{g}^{\prime}=\mathfrak{s}^{\perp}=: \mathfrak{g} \ominus \mathfrak{s}$ contains the "proper" gauge transformations, acting freely near $C$. If $\left(n_{1}, \ldots, n_{n}\right)$ is a partition of the integer $n N$ which does not contain trivial representations of $S U(2)$ (no fluxons), then $\mathfrak{g}^{\prime}$ is the tangent space to the corresponding critical surface $\mathcal{C}_{\left(n_{1}, 1\right), \ldots,\left(n_{n}, 1\right)} \subset \mathcal{O}$,

$$
\mathcal{C}_{\left(n_{1}, 1\right), \ldots,\left(n_{n}, 1\right)} \cong U(n N) / S
$$

where $S=\exp (\mathfrak{s})$.

We claim that the subspaces $\mathcal{J}(\mathfrak{g})$ and $\mathfrak{g}$ are linearly independent. For this, assume to the contrary that $\mathcal{J}(\mathfrak{g})$ and $\mathfrak{g}$ are linearly dependent, i.e. $\mathcal{J}(g) \in \mathfrak{g}$ for some $g \in \mathfrak{g}$. This implies that $\left[C_{i}, g\right]=0$, and therefore $\left[C_{0}^{2}, g\right]=0$ due to $(2.11)$. Restricting attention to critical points $C$ for which the spectrum of $C_{0}$ is non-negative (the others being strongly suppressed at large $N$ ), this implies that $g$ commutes with the spectral projectors of $C_{0}$, and hence also with $C_{0}$ itself. Together with $\left[C_{i}, g\right]=0$ it follows that $\mathcal{J}(g)=0$. However, $\mathcal{J}(\mathfrak{g})$ and $\mathfrak{g}$ need not be orthogonal subspaces. 
Generically one then has

$$
\mathcal{J}^{2}(\mathfrak{g})+\mathcal{J}(\mathfrak{g}) \subset T_{C} \mathcal{O}
$$

The two subspaces are not orthogonal in general, since for $g_{1}, g_{2} \in \mathfrak{g}$ one can compute the inner product

$$
\begin{aligned}
\operatorname{Tr}\left(\mathcal{J}^{2}\left(g_{1}\right) \mathcal{J}\left(g_{2}\right)\right) & =\operatorname{Tr}\left(g_{1} \mathcal{J}\left(g_{2}\right)\right) \\
& =-\frac{\mathrm{i}}{N} \operatorname{Tr}\left(C\left[g_{1}, g_{2}\right]\right) \\
& =-\frac{1}{N}\left\langle\omega, V_{g_{1}} \wedge V_{g_{2}}\right\rangle=\frac{\mathrm{i}}{N} \operatorname{Tr}\left(g_{1}\left[C_{0}, g_{2}\right]\right)
\end{aligned}
$$

which is non-vanishing in general. For the vacuum solution with $C_{0}=\frac{1}{2} \mathbb{1}_{n N}$, it follows from this expression that the subspaces are indeed orthogonal, and hence $\mathcal{J}^{2}(\mathfrak{g}) \oplus \mathcal{J}(\mathfrak{g}) \subset T_{C} \mathcal{O}$. In fact, one has

$$
\mathcal{J}^{2}(\mathfrak{g}) \oplus \mathcal{J}(\mathfrak{g})=T_{C} \mathcal{O} \quad \text { if } \quad C_{0}=\frac{1}{2} \mathbb{1}_{n N}
$$

which provides a useful description of the local geometry near the global minimum. To see (3.30), note first that in the abelian case $n=1$ one has $\mathfrak{s}=\mathfrak{u}(1)$, and $(3.30)$ then follows since $\operatorname{dim}(\mathcal{O})=$ $2\left(N^{2}-1\right)=2 \operatorname{dim}\left(\mathfrak{g}^{\prime}\right)$. In the nonabelian case, for the vacuum state the gauge stabilizer $\mathfrak{s} \cong \mathfrak{u}(n)$ has dimension $n^{2}$ and hence $\operatorname{dim}\left(\mathcal{J}^{2}\left(\mathfrak{g}^{\prime}\right) \oplus \mathcal{J}\left(\mathfrak{g}^{\prime}\right)\right)=2 n^{2} N^{2}-2 n^{2}=\operatorname{dim}(\mathcal{O})$.

In general, the subspaces $\mathcal{J}(\mathfrak{g})=\mathcal{J}(\mathfrak{g} \ominus \mathfrak{s})$ and $\mathcal{J}^{2}(\mathfrak{g})$ are not linearly independent, and we can define

$$
E_{0}:=\mathcal{J}(\mathfrak{g}) \cap \mathcal{J}^{2}(\mathfrak{g})
$$

which is generically a non-trivial subspace. Define also the subspaces $\mathfrak{h}, \tilde{\mathfrak{h}} \subset \mathfrak{g} \ominus \mathfrak{s}$ with the properties that

$$
\mathcal{J}(\mathfrak{h})=E_{0}=\mathcal{J}^{2}(\tilde{\mathfrak{h}}) .
$$

Since $\mathcal{J}^{2}(\mathfrak{h})=-\mathcal{J}(\tilde{\mathfrak{h}})$ implies that $\mathfrak{h} \subset \tilde{\mathfrak{h}} \subset \mathfrak{h}$, we have

$$
\mathfrak{h}=\tilde{\mathfrak{h}} \quad \text { and } \quad \mathcal{J}\left(E_{0}\right)=E_{0} .
$$

We can accordingly decompose the gauge algebra $\mathfrak{g}$ into orthogonal subspaces as

$$
\mathfrak{g}=\mathfrak{g}_{1} \oplus \mathfrak{h} \oplus \mathfrak{s}
$$

Since $\mathcal{J}: \mathfrak{h} \rightarrow E_{0}$ is a bijection, there is a unique map

$$
j: \mathfrak{h} \longrightarrow \mathfrak{h} \quad \text { with } \quad \mathcal{J}^{2}(h)=\mathcal{J}(j(h))
$$

for all $h \in \mathfrak{h}$ which satisfies $j^{2}=-\mathbb{1}_{n N}$. Similarly, in order to span the entire tangent space at $C \in \mathcal{O}$ we generally have to introduce another subspace $E_{1}$, with $\mathcal{J}\left(E_{1}\right)=E_{1}$, which gives the general decomposition

$$
\mathcal{J}(\mathfrak{g} \ominus \mathfrak{h}) \oplus \mathcal{J}^{2}(\mathfrak{g} \ominus \mathfrak{h}) \oplus E_{0} \oplus E_{1}=T_{C} \mathcal{O}
$$

\subsection{Explicit decomposition at Yang-Mills critical surfaces}

We will now provide an explicit description of the various subspaces appearing in the decomposition of the tangent space (3.36). Consider the Yang-Mills critical surfaces $\mathcal{C}_{\left(n_{1}, 1\right), \ldots,\left(n_{n}, 1\right)}$ and suppose first that $n_{1} \neq \cdots \neq n_{n}$ are all distinct integers, corresponding to a completely nondegenerate solution. The elements $\phi$ of the subspace (3.25) satisfy $[C, \phi]=0$. This implies that $\phi$ respects the block decomposition described by the given partition $\left(n_{1}, \ldots, n_{n}\right)$, and is therefore proportional to 
$\mathbb{1}_{n_{i}}$ on each block. These are thus $\mathfrak{u}(1)^{n}$ degrees of freedom. If some $n_{i}$ are degenerate, this space is enhanced to

$$
\mathfrak{s}=\mathfrak{u}\left(k_{1}\right) \times \cdots \times \mathfrak{u}\left(k_{l}\right)
$$

for a critical surface with $C=\bigoplus_{i} C\left(n_{i}\right) \otimes \mathbb{1}_{k_{i}}$ and $n_{i}$ all distinct. For the vacuum this is $\mathfrak{u}(n)$, corresponding to the maximally degenerate solution, as in Section 3.3 above.

We wish to work out the map $\mathcal{J}$ explicitly. For this, we decompose

$$
\phi=\left(\begin{array}{ccc}
\phi_{11} & \phi_{12} & \cdots \\
\phi_{21} & \phi_{22} & \cdots \\
\cdots & \cdots & \ddots
\end{array}\right)
$$

where $\phi_{i j} \in\left(n_{i}\right) \otimes\left(n_{j}\right)$ and as before $(p)$ denotes the $p$-dimensional irreducible representation of $S U(2)$. In the degenerate case, there is another factor corresponding to $\mathfrak{u}\left(k_{j}\right)$. The nonorthogonality of $\mathcal{J}(\mathfrak{g})$ and $\mathcal{J}^{2}(\mathfrak{g})$ in $(3.29)$ is now easily understood as being simply due to the different $\mathfrak{u}(1)$ charges between the $S U(2)$ sectors of $\mathfrak{s}$. Since $\left[C, C_{0}\right]=0$ at the Yang-Mills critical surfaces, one has $\mathcal{J}\left(\left[C_{0}, \phi\right]\right)=\left[C_{0}, \mathcal{J}(\phi)\right]$. Thus the hermitian operator

$$
\left(\operatorname{ad}_{\mathrm{i} C_{0}}\right)_{i j}=\mathrm{i} C_{0}\left(n_{i}\right)-\mathrm{i} C_{0}\left(n_{j}\right)=\mathrm{i} \frac{N}{2} \frac{n_{j}-n_{i}}{n_{i} n_{j}}=: \mathrm{i} c_{i j}
$$

acting on $\phi_{i j} \in\left(n_{i}\right) \otimes\left(n_{j}\right)$ commutes with $\mathcal{J}$. This implies that we can decompose the subspaces in (3.36) such as $\mathcal{J}(\mathfrak{h})=\mathcal{J}^{2}(\mathfrak{h})=E_{0}$ into irreducible representations of the operator $\operatorname{ad}_{\mathrm{i} C_{0}}$, i.e. into the various $\mathfrak{u}(1)$ blocks. Restricted to the diagonal blocks $C_{0}\left(n_{i}\right)$ is proportional to the unit matrix $\mathbb{1}_{n_{i}}$, so that $\operatorname{Tr}\left(\mathcal{J}\left(g_{1}\right) \mathcal{J}^{2}\left(g_{2}\right)\right)=0$ there as for the vacuum.

\section{Global SU(2) symmetry}

To proceed further, we need to exploit an additional symmetry that we have neglected so far, the global rotation group $S U(2)$. Recall from Section 3.1 above that each saddle-point defines a representation of $S U(2)$ acting on the representation space $V \cong \mathbb{C}^{n N}$ as (3.4), and trivially on potential fluxon components. In the abelian case $n=1$, this induces via the adjoint action the rotations of functions $f \mapsto J_{i} f=\left[L_{i}, f\right]$ in $S_{N}^{2} \cong V \otimes \bar{V}$, but it is a somewhat different symmetry for the nonabelian instantons. Let us decompose $V$ into irreducible representations as

$$
V=\bigoplus_{i=1}^{n}\left(n_{i}\right)
$$

This representation can be extended to the module $V \otimes \mathbb{C}^{2}$ for the action of the operators

$$
J_{i}=L_{i}+\frac{1}{2} \sigma^{i}
$$

which by construction commute with $C$,

$$
\left[J_{i}, C\right]=0,
$$

on the critical surfaces. This follows from the fact that $C_{i} \otimes \sigma^{i}$ is an intertwiner for the action of $J_{i}$ on

$$
V \otimes \mathbb{C}^{2}=\left(\bigoplus_{i=1}^{n}\left(n_{i}+1\right)\right) \oplus\left(\bigoplus_{i=1}^{n}\left(n_{i}-1\right)\right)=: V^{+} \oplus V^{-}
$$


and $C$ has eigenvalues $\pm \frac{N}{2}$ on the component subspaces $V^{ \pm}$. This enables one to decompose $C$ further using the projectors $\Pi_{i}^{ \pm}$onto the irreducible representations $\left(n_{i} \pm 1\right)$ with

$$
\left[C, \Pi_{i}^{ \pm}\right]=0,
$$

and the constrained covariant coordinates take the simple form

$$
C=\frac{N}{2}\left(\begin{array}{cc}
\bigoplus_{i=1}^{n} \Pi_{i}^{+} & 0 \\
0 & -\bigoplus_{i=1}^{n} \Pi_{i}^{-}
\end{array}\right) .
$$

In particular, since $C_{0} \otimes \sigma^{0}$ is two-fold degenerate it follows that

$$
C_{0} \otimes \sigma^{0}=\left(\begin{array}{cc}
\bigoplus_{i=1}^{n} C_{0}\left(n_{i}\right) \Pi_{i}^{+} & 0 \\
0 & \bigoplus_{i=1}^{n} C_{0}\left(n_{i}\right) \Pi_{i}^{-}
\end{array}\right)
$$

separates the explicit blocks according to (3.39).

The complex structure map $\mathcal{J}$ respects this $S U(2)$ symmetry,

$$
\left[J_{i}, \mathcal{J}\right]=0,
$$

which enables one to decompose the tangent space $T_{C} \mathcal{O}$ into irreducible representations of the $S U(2)$ isometry group. With respect to the block decomposition $(3.43)$, the subspace $\operatorname{ker}(\mathcal{J}) \subset \mathfrak{u}(\mathcal{N})$ consists of block diagonal operators while $T_{C} \mathcal{O}$ consists of block off-diagonal operators, and the action of $\mathcal{J}$ on tangent vectors is given explicitly by

$$
\mathcal{J}\left(\begin{array}{cc}
0 & X \\
X^{\dagger} & 0
\end{array}\right)=\left(\begin{array}{cc}
0 & \mathrm{i} X \\
-\mathrm{i} X^{\dagger} & 0
\end{array}\right)
$$

This is the obvious complex structure on $T_{C} \mathcal{O}$ compatible with the action of the isometry group.

The decomposition of the tangent space $T_{C} \mathcal{O}$ into irreducible representations of $S U(2)$ is now provided by

$$
T_{C}^{-} \mathcal{O} \cong\left(\bigoplus_{i=1}^{n}\left(n_{i}+1\right)\right) \otimes\left(\bigoplus_{j=1}^{n}\left(n_{j}-1\right)\right)=\bigoplus_{i, j=1}^{n}\left(n_{i}+1\right) \otimes\left(n_{j}-1\right),
$$

where $T_{C}^{ \pm} \mathcal{O}:=\left.T_{C} \mathcal{O}\right|_{V^{ \pm}}$corresponds to the upper-right respectively lower-left blocks in (3.48), and the different sectors $(i, j)$ are separated by the eigenvalues of the operator adi $C_{0}$ in the irreducible case. Note in particular that the lowest spin component in the Clebsch-Gordan decomposition of $\left(n_{i}+1\right) \otimes\left(n_{i}-1\right)$ is a spin one field as appropriate for gauge fields. This implies $\mathcal{J}\left(\mathfrak{g}_{0}\right)=0$, where $\mathfrak{g}_{0}$ is the subspace of $S U(2)$ singlet components of $\mathfrak{g}$, and in fact $\mathfrak{g}_{0}=\mathfrak{s}$ by Schur's lemma.

\section{Global minimum}

Consider first the vacuum surface $\mathcal{C}_{(N, 1), \ldots,(N, 1)}$. Compare the $S U(2)$-invariant decomposition of the gauge algebra $\mathfrak{g}$, given by

$$
\begin{aligned}
\mathfrak{g} & \cong(N) \otimes(N) \otimes \mathfrak{u}(n) \\
& =((1) \oplus(3) \oplus \cdots \oplus(2 N-1)) \otimes \mathfrak{u}(n)=((1) \oplus(N+1) \otimes(N-1)) \otimes \mathfrak{u}(n),
\end{aligned}
$$

with (3.49) in the degenerate case $C_{0}=\frac{1}{2} \mathbb{1}_{n N}$. It follows that the image of $\mathcal{J}(\mathfrak{g})$ indeed covers all modes of $T_{C} \mathcal{O}$, and the complexification is achieved by adding $\mathcal{J}^{2}(\mathfrak{g})$. This gives another proof of the decomposition (3.30). The singlet subspace of (3.50) is $\mathfrak{g}_{0}=(1) \otimes \mathfrak{u}(n) \cong \mathfrak{u}(n)=\mathfrak{s}$. 
Now consider a generic, completely non-degenerate critical surface $\mathcal{C}_{\left(n_{1}, 1\right), \ldots,\left(n_{n}, 1\right)}$, and the corresponding decomposition of $T_{C} \mathcal{O}=T_{C}^{-} \mathcal{O} \oplus T_{C}^{+} \mathcal{O}$ given by (3.49). The different sectors $(i, j)$ are distinguished by the eigenvalues of the operator $\operatorname{ad}_{\mathrm{i} C_{0}}$. Hence we can pick some fixed pair $n_{i}>n_{j}$, and decompose

$$
\left(n_{i}+1\right) \otimes\left(n_{j}-1\right) \cong\left(\left|n_{i}-n_{j}\right|+3\right)_{\mathrm{i} c_{i j}} \oplus\left(\left|n_{i}-n_{j}\right|+5\right)_{\mathrm{i} c_{i j}} \oplus \cdots \oplus\left(n_{i}+n_{j}-1\right)_{\mathrm{i} c_{i j}} \subset T_{C} \mathcal{O}
$$

which has eigenvalue given by (3.39) as indicated by the subscripts. Similarly, one has

$$
\left(n_{j}+1\right) \otimes\left(n_{i}-1\right) \cong\left(\left|n_{i}-n_{j}\right|-1\right)_{\mathrm{i} c_{j i}} \oplus\left(\left|n_{i}-n_{j}\right|+1\right)_{\mathrm{i} c_{j i}} \oplus \cdots \oplus\left(n_{i}+n_{j}-1\right)_{\mathrm{i} c_{j i}} \subset T_{C} \mathcal{O}
$$

(where (0) is omitted) with ad $\mathrm{i}_{0}$ eigenvalue $\mathrm{i} c_{j i}=-\mathrm{i} c_{i j}$. The corresponding conjugate matrix decompositions $\left(n_{j}-1\right) \otimes\left(n_{i}+1\right)$ and $\left(n_{i}-1\right) \otimes\left(n_{j}+1\right)$ are determined by hermiticity. They are given respectively by (3.51) with eigenvalue i $c_{j i}=-\mathrm{i} c_{i j}$ and by (3.52) with eigenvalue i $c_{i j}$.

We denote the tangent space decomposition (3.49) determined by (3.51) and (3.52) as

$$
T_{C} \mathcal{O}:=\bigoplus_{i, j=1}^{n} \mathbb{C}\left|n ; n_{i}+1, n_{j}-1 ; \mathrm{i} c_{i j}, l\right\rangle_{T_{C} \mathcal{O}}
$$

where $n$ denotes the dimension of $(n)$, and we will drop its magnetic quantum number $l$ from now on. This defines a natural basis for $T_{C} \mathcal{O}$, in which the action of $\mathcal{J}$ is given by block-wise multiplication with

$$
\mathcal{J}=\sigma^{2}=\left(\begin{array}{cc}
0 & \mathrm{i} \\
-\mathrm{i} & 0
\end{array}\right)
$$

as in (3.48), and the action of $\operatorname{ad}_{\mathrm{i} C_{0}}$ by

$$
\left(\operatorname{ad}_{\mathrm{i} C_{0}}\right)_{i j}=\left|c_{i j}\right|\left(\begin{array}{cc}
0 & \sigma^{2} \\
\sigma^{2} & 0
\end{array}\right)
$$

since its sign depends on $n_{i} \gtrless n_{j}$. In particular, by virtue of (3.23) the tangent space $T_{C} \mathcal{O}$ is naturally a symplectic vector space with symplectic form of type $(1,1)$ with respect to the complex structure $\mathcal{J}$. This construction thereby defines a local symplectic model for the neighbourhood of the Yang-Mills critical point $C$ in the Kähler manifold $\mathcal{O}$. In the next section this model space will be used to evaluate fluctuation integrals over tubular neighbourhoods of the critical surfaces. In particular, all pertinent one-forms can be explicitly evaluated on $T_{C} \mathcal{O}$ by using the explicit expressions for $C$ and $C_{0}$ in (3.45) and (3.46).

Let us now look at the $S U(2)$-invariant decomposition of the gauge algebra $\mathfrak{g}$ given by

$$
\begin{aligned}
\mathfrak{g} & \cong \bigoplus_{i, j=1}^{n}\left(n_{i}\right) \otimes\left(n_{j}\right) \\
& =\bigoplus_{i, j=1}^{n}\left(\left(\left|n_{i}-n_{j}\right|+1\right) \oplus\left(\left|n_{i}-n_{j}\right|+3\right) \oplus \cdots \oplus\left(n_{i}+n_{j}-1\right)\right)=: \bigoplus_{i, j=1}^{n} \mathbb{C}\left|n ; n_{i}, n_{j} ; \mathrm{i} c_{i j}\right\rangle_{\mathfrak{g}} .
\end{aligned}
$$

This can be compared with the $S U(2)$-invariant decomposition of the tangent space $T_{C} \mathcal{O}$ in (3.53) above, whose higher modes match perfectly with those of $\mathfrak{g}$ except for a doubling due to the complex structure $\mathcal{J}$. There is, however, some mismatch in the low lying modes. In particular, $T_{C} \mathcal{O}$ contains the extra subspace

$$
E_{1}:=\bigoplus_{i>j} \mathbb{C}\left|n_{i}-n_{j}-1 ; n_{j}+1, n_{i}-1 ;-\mathrm{i} c_{i j}\right\rangle_{T_{C} \mathcal{O}}
$$


which is not contained in $\mathcal{J}(\mathfrak{g})$. On the other hand, the modes in the subspace

$$
E_{0}:=\bigoplus_{i>j} \mathbb{C}\left|n_{i}-n_{j}+1 ; n_{j}+1, n_{i}-1 ;-\mathrm{i} c_{i j}\right\rangle_{T_{C} \mathcal{O}}
$$

occur only once in $T_{C} \mathcal{O}$, which means that they are already spanned by the image $\mathcal{J}(\mathfrak{g})$ since $\mathcal{J} \neq 0$ on the non-trivial modes. This implies that $E_{0}=\mathcal{J}\left(E_{0}\right)=\mathcal{J}(\mathfrak{h})$ where

$$
\mathfrak{h}=\bigoplus_{i \neq j} \mathbb{C}|| n_{i}-n_{j}\left|+1 ; n_{i}, n_{j} ; \mathrm{i} c_{i j}\right\rangle_{\mathfrak{g}}
$$

The linear independence of the subspaces $\mathcal{J}(\mathfrak{g} \ominus \mathfrak{h})$ and $\mathcal{J}^{2}(\mathfrak{g} \ominus \mathfrak{h})$ follows from the explicit embedding $T_{C} \mathcal{O} \hookrightarrow \mathfrak{u}(\mathcal{N})$ given below. Therefore $\mathcal{J}(\mathfrak{g} \ominus \mathfrak{h}) \oplus \mathcal{J}^{2}(\mathfrak{g} \ominus \mathfrak{h})$ spans the entire tangent space $T_{C} \mathcal{O}$ except for the subspace $E_{1}$, which gives the decomposition (3.36) with the various subspaces now explicitly identified. We have $\mathcal{J}\left(E_{0}\right)=E_{0}$ and $\mathcal{J}\left(E_{1}\right)=E_{1}$, with the action of $\mathcal{J}$ given by diagonal eigenvalues $\pm \mathrm{i}$ on the two components in (3.58) and (3.57). On the remaining space $T_{C} \mathcal{O} \ominus E_{0} \ominus E_{1}$ the action of $\mathcal{J}$ is obtained by exchanging the two components in (3.36).

To complete this analysis, we need to explicitly embed $T_{C} \mathcal{O}$ into the space $\mathfrak{u}(\mathcal{N})$, which admits the $S U(2)$-invariant decomposition

$$
\begin{array}{r}
\mathfrak{u}(\mathcal{N}) \cong \mathfrak{g} \otimes((2) \otimes(2))=\bigoplus_{i, j=1}^{n}\left(\left(n_{i}+1\right) \otimes\left(n_{j}+1\right) \oplus\left(n_{i}-1\right) \otimes\left(n_{j}-1\right)\right. \\
\left.\oplus\left(n_{i}+1\right) \otimes\left(n_{j}-1\right) \oplus\left(n_{i}-1\right) \otimes\left(n_{j}+1\right)\right)
\end{array}
$$

corresponding to (3.43). Since we know the action of $\mathcal{J}$ on the rhs, we can determine the map

$$
\mathcal{J}: \mathfrak{g} \longrightarrow T_{C} \mathcal{O} \hookrightarrow \mathfrak{g} \otimes((2) \otimes(2))
$$

using

$\mathcal{J}\left(\left|n ; n_{i}, n_{j} ; \mathrm{i} c_{i j}\right\rangle_{\mathfrak{g}}\right)=\sum_{k, l=1}^{n} \mathcal{J}\left(\left|n ; n_{k}+1, n_{l}-1 ; \mathrm{i} c_{k l}\right\rangle_{T_{C} \mathcal{O}}\right) T_{C} \mathcal{O}\left\langle n ; n_{k}+1, n_{l}-1 ; \mathrm{i} c_{k l} \mid n ; n_{i}, n_{j} ; \mathrm{i} c_{i j}\right\rangle_{\mathfrak{g}}+h . c$.

The non-vanishing inner products in this expression can be written in terms of Wigner $6 j$-symbols for the group $S U(2)$, which are known explicitly. This also enables one to compute the projection

$$
\Pi_{0}: T_{C} \mathcal{O} \longrightarrow \mathfrak{g}, \quad V_{0} \otimes \sigma^{0}+V_{i} \otimes \sigma^{i} \longmapsto V_{0}
$$

as

$$
\Pi_{0}\left|n ; n_{i}+1, n_{j}-1 ; \mathrm{i} c_{i j}\right\rangle_{T_{C} \mathcal{O}}=\sum_{k, l=1}^{n}\left|n ; n_{k}, n_{l} ; \mathrm{i} c_{k l}\right\rangle_{\mathfrak{g} \mathfrak{g}}\left\langle n ; n_{k}, n_{l} ; \mathrm{i} c_{k l} \mid n ; n_{i}+1, n_{j}-1 ; \mathrm{i} c_{i j}\right\rangle_{T_{C} \mathcal{O}} .
$$

In the basis (3.36), one has the useful explicit formula

$$
\Pi_{0} \mathcal{J}(\mathfrak{g})=\operatorname{ad}_{\mathrm{i} C_{0}}(\mathfrak{g})
$$

which is of order $\frac{1}{N}$ and can also be used for $E_{0}$, while $\Pi_{0} \mathcal{J}^{2}(\mathfrak{g})$ is of order $\frac{1}{N^{2}}$ and $\Pi_{0}\left(E_{1}\right)=\{0\}$. 


\section{General solutions}

The case where some of the irreducible representations $\left(n_{i}\right)$ have multiplicity $k_{i}>1$ is a combination of the structures above for the vacuum state and for the nondegenerate case. Now the basis (3.53) acquires additional labelling reflecting the $\mathfrak{u}\left(k_{i}\right)$ degrees of freedom, and it takes the symbolic form

$$
T_{C} \mathcal{O}=\bigoplus_{i, j=1}^{l} \mathbb{C}\left|n ;\left(n_{i}+1, a_{i}\right),\left(n_{j}-1, a_{j}\right) ; \mathrm{i} c_{i j}\right\rangle_{T_{C} \mathcal{O}}
$$

In particular, one can now easily compute the symplectic form on $T_{C} \mathcal{O}$ using (3.23). It is essentially given by the complex structure $\mathcal{J}$.

\subsection{Fluctuations around the critical surfaces}

We conclude this section with a summary of the salient features of the decompositions in Sections 3.3 and 3.4 above, as pertaining to how they will be exploited in the next section to evaluate fluctuation integrals over the local neighbourhoods of Yang-Mills critical points. Recall that globally the critical surface (with no fluxons) through some critical point $C$ is given by the space of gauge transformations acting on $C$, as in (3.27). Its tangent space is embedded locally as

$$
T_{C} \mathcal{C}_{\left(n_{1}, s_{1}\right), \ldots,\left(n_{k}, s_{k}\right)}=\mathcal{J}(\mathfrak{g} \ominus \mathfrak{s}) \subset T_{C} \mathcal{O}
$$

which can be determined explicitly using (3.62). Recall also that the gauge stabilizer $\mathfrak{s}$ of $C$ consists of the $S U(2)$ singlets in $\mathfrak{g}$. It is given by $\mathfrak{s} \cong \mathfrak{u}(n)$ for the vacuum, and $\mathfrak{s} \cong \mathfrak{u}(1)^{n}$ for completely irreducible saddle-points. In particular, $\mathfrak{s}$ is never trivial, quite unlike the situation in ordinary two-dimensional Yang-Mills theory [27]. The global symmetry cannot be disentangled in the noncommutative case, and the nonabelian localization even at the global minimum is akin to that at higher critical points of two-dimensional Yang-Mills theory or more precisely at the flat connections of Chern-Simons gauge theory on a Seifert fibration [27]. The non-trivial part of the localization at higher critical points will therefore be given by fluctuation integrals over the spaces $E_{0}, E_{1}$ and $\mathfrak{s}$. The only effect of the remaining part $\mathcal{J}(\mathfrak{g} \ominus \mathfrak{h}) \oplus \mathcal{J}^{2}(\mathfrak{g} \ominus \mathfrak{h})$ will be to induce normalization terms as for the vacuum critical point. In particular, the subspaces $\mathcal{J}(\mathfrak{g} \ominus \mathfrak{s})$ and $\mathcal{J}^{2}(\mathfrak{g} \ominus \mathfrak{s})$ locally model the tangent space $T_{C} \mathcal{O}$ near the vacuum.

To understand the physical meaning of the subspace $E_{1}$, note that the gauge field strength

remains constant for variations along $\phi=X \in E_{1}$, since $\left.\delta C_{0}\right|_{E_{1}}=i[C, \phi]_{0} \in \Pi_{0}\left(E_{1}\right)=\{0\}$. Let us compute the second order variation of the Yang-Mills action, given by

$$
\begin{aligned}
\operatorname{Tr}\left(C_{0} \delta^{2} C_{0}\right) & =-\operatorname{Tr}\left(C_{0}[[C, \phi], \phi]\right) \\
& =\operatorname{Tr}\left(\left[C_{0}, \phi\right][C, \phi]\right)=-N \operatorname{Tr}\left(\operatorname{ad}_{\mathrm{i} C_{0}}(\phi) \mathcal{J}(\phi)\right) .
\end{aligned}
$$

Restricting to fluctuations $\phi=X \in E_{1}$ with respect to the decomposition (3.57) one has

$$
\left.\operatorname{Tr}\left(C_{0} \delta^{2} C_{0}\right)\right|_{E_{1}}=-N \sum_{i>j} \operatorname{Tr}\left(\operatorname{ad}_{\mathrm{i} C_{0}}\left(X_{j i}^{\dagger}\right) \mathcal{J}\left(X_{j i}\right)\right)=-2 N \sum_{i>j}\left|c_{i j}\right| \operatorname{Tr}\left(X_{j i}^{\dagger} X_{j i}\right)
$$

by using the actions (3.54) and (3.55), cf. (4.85). For the maximally nondegenerate saddle-points, this fluctuation is thus negative, demonstrating that the two-dimensional instantons on the fuzzy sphere $S_{N}^{2}$ are generically unstable. On the other hand, since the subspace $E_{0}=\mathcal{J}(\mathfrak{h})$ is obtained through gauge transformations, it produces flat directions for the Yang-Mills action. 


\section{Nonabelian localization}

This section is the crux of the present paper, wherein we shall derive the semiclassical expansion of the partition function for Yang-Mills theory on the fuzzy sphere $S_{N}^{2}$ and show that it agrees with the known instanton expansion of quantum gauge theory on $S^{2}$ in the classical limit $N \rightarrow \infty$. We will begin by describing the nonabelian localization principle, adapted to our specific gauge theory. We will then explicitly evaluate the contributions from two extreme classes of Yang-Mills critical points, the vacuum and the maximally irreducible solutions, and show that they give the expected contributions to the path integral at large $N$. The intermediate contributions from degenerate solutions, which we do not treat in detail here, are somewhat more involved but can in principle be evaluated using our techniques. The contribution from the vacuum to the partition function could be expressed in terms of the abstract cohomological formula of [18] given by intersection pairings on the vacuum moduli space, or by using the more explicit residue formula of [23]. The contributions from some higher unstable critical points to the nonabelian localization formula are formally described in $[24,26,27]$, but the general cases that we need (including reducible saddle points) are not explicitly treated in full generality. Here we will directly evaluate, following [27], the explicit quantum fluctuation integrals near the critical points using the local symplectic geometry of the previous section.

\subsection{Equivariant cohomology and the localization principle}

The goal of this section is to compute the partition function of quantum Yang-Mills theory on the fuzzy sphere defined by the action (2.25) on the configuration space (2.13) of gauge fields. After an irrelevant shift of the covariant coordinates (2.8) which is equivalent to working with the reduced Yang-Mills action (3.15), it is defined by

$$
\begin{aligned}
Z & :=\frac{1}{\operatorname{vol}(G)}\left(\frac{g}{4 \pi N}\right)^{\operatorname{dim}(G) / 2} \int_{\mathcal{O}} \mathrm{d} C \exp \left(-\frac{N}{g} \operatorname{Tr}\left(C_{0}^{2}\right)\right) \\
& =\frac{1}{\operatorname{vol}(G)}\left(\frac{g^{\prime}}{2 \pi}\right)^{\operatorname{dim}(G) / 2} \int_{\mathcal{O}} \exp \left(\omega-\frac{1}{2 g^{\prime}} \operatorname{Tr}\left(C_{0}^{2}\right)\right)
\end{aligned}
$$

where we have used the fact that the symplectic volume form $\omega^{d} / d$ !, with $d:=\operatorname{dim}_{\mathbb{C}}(\mathcal{O})$, defines the natural gauge invariant measure on $\mathcal{O}$ provided by the Cartan-Killing riemannian volume form (up to some irrelevant normalization). This follows from the fact that the natural invariant metric on $\mathcal{O}$ is a Kähler form. We have divided by the volume of the gauge group $G=U(n N)$ with respect to the invariant Cartan-Killing form and by another normalization factor for later convenience, and also introduced the rescaled gauge coupling

$$
g^{\prime}=\frac{g}{2 N} .
$$

We will now describe, following [18,27], how the technique of nonabelian localization can be applied to evaluate the symplectic integral (4.1) exactly.

We begin by using a gaussian integration to rewrite (4.1) as

$$
Z=\frac{1}{\operatorname{vol}(G)} \int_{\mathfrak{g} \times \mathcal{O}}\left[\frac{\mathrm{d} \phi}{2 \pi}\right] \exp \left(\omega-\mathrm{i} \operatorname{Tr}\left(C_{0} \phi\right)-\frac{g^{\prime}}{2} \operatorname{Tr}\left(\phi^{2}\right)\right),
$$

where the euclidean measure for integration over the gauge algebra $\phi \in \mathfrak{g}=\mathfrak{u}(n N)$ is determined by the invariant Cartan-Killing form. Since the moment map for the $G$-action on $\mathcal{O}$ is given by (2.40), by (2.38) we have

$$
\mathrm{d} \operatorname{Tr}\left(C_{0} \phi\right)=-\iota_{V_{\phi}} \omega
$$


Introduce the BRST operator

$$
Q=\mathrm{d}-\mathrm{i} \iota_{V_{\phi}},
$$

where $\mathrm{d}$ is the exterior derivative on $\Omega(\mathcal{O})$ and the contraction $\iota_{V_{\phi}}$ acts trivially on $\phi$. It preserves the gradation if one assigns charge +2 to the elements $\phi$ of $\mathfrak{g}$, and it satisfies

$$
Q^{2}=-\mathrm{i}\left\{\mathrm{d}, \iota_{V_{\phi}}\right\}=-\mathrm{i} \mathcal{L}_{V_{\phi}}
$$

where $\mathcal{L}_{V_{\phi}}$ is the Lie derivative along the vector field $V_{\phi}$. Thus $Q^{2}=0$ exactly on the space

$$
\Omega_{G}(\mathcal{O}):=(\mathbb{C}[[\mathfrak{g}]] \otimes \Omega(\mathcal{O}))^{G}
$$

consisting of gauge invariant differential forms on $\mathcal{O}$ which take values in the ring of symmetric functions on the Lie algebra $\mathfrak{g}$.

By construction one has

$$
Q\left(\omega-\mathrm{i} \operatorname{Tr}\left(C_{0} \phi\right)\right)=0
$$

using (2.34) and (4.4), and

$$
Q \operatorname{Tr}\left(\phi^{2}\right)=0 \text {. }
$$

Therefore, the integrand of the partition function (4.3) defines a $G$-equivariant cohomology class in $H_{G}(\mathcal{O})$, and the value of $Z$ depends only on this class. The integral of any $Q$-exact equivariant differential form in $\Omega_{G}(\mathcal{O})$ over $\mathfrak{g} \times \mathcal{O}$ is clearly 0 , as is the integral of any $\iota_{V_{\phi}}$-exact form even if its argument is not gauge invariant. Thus $Z$ is unchanged by adding any $Q$-exact form to the action, which will fix a gauge for the localization. Hence we can replace it by

$$
Z=\frac{1}{\operatorname{vol}(G)} \int_{\mathfrak{g} \times \mathcal{O}}\left[\frac{\mathrm{d} \phi}{2 \pi}\right] \exp \left(\omega-\mathrm{i} \operatorname{Tr}\left(C_{0} \phi\right)-\frac{g^{\prime}}{2} \operatorname{Tr}\left(\phi^{2}\right)+t Q \alpha\right),
$$

which is independent of $t \in \mathbb{R}$ for any $G$-invariant one-form $\alpha$ on $\mathcal{O}$, where

$$
Q \alpha=\mathrm{d} \alpha-\mathrm{i}\left\langle\alpha, V_{\phi}\right\rangle .
$$

The independence of (4.10) on the particular representative $\alpha \in \Omega(\mathcal{O})^{G}$ of its equivariant cohomology class will play a crucial role in our evaluation of the partition function.

Expanding the integrand of (4.10) by writing $\exp (t \mathrm{~d} \alpha)$ as a polynomial in $t$ and using the fact that the configuration space $\mathcal{O}$ is compact, it follows that for $t \rightarrow \infty$ the integral localizes at the stationary points of $\left\langle\alpha, V_{\phi}\right\rangle$ in $\mathfrak{g} \times \mathcal{O}$. By writing $V_{\phi}=V_{a} \phi^{a}$, where $\phi^{a}$ is an orthonormal basis of $\mathfrak{g}^{\vee}$, we have $\left\langle\alpha, V_{\phi}\right\rangle=\left\langle\alpha, V_{a}\right\rangle \phi^{a}$ and the critical points are thus determined by the equations

$$
\begin{aligned}
\left\langle\alpha, V_{a}\right\rangle & =0, \\
\phi^{a} \mathrm{~d}\left\langle\alpha, V_{a}\right\rangle & =0 .
\end{aligned}
$$

Since (4.13) is invariant under rescaling of $\phi$ and the Lie algebra $\mathfrak{g}$ is contractible, the homotopy type of the space of solutions in $\mathfrak{g} \times \mathcal{O}$ is unchanged by restricting to $\phi=0$ and the saddle-points reduce to the zeroes of $\left\langle\alpha, V_{a}\right\rangle$ in $\mathcal{O}$.

Given the reduced Yang-Mills function (3.15), let us consider explicitly the invariant one-form $\alpha$ given by $[27,32]$

$$
\alpha=-\mathrm{i} \operatorname{Tr}\left(C_{0}[C, \mathrm{~d} C]_{0}\right)=g^{\prime} \mathcal{J}\left(\mathrm{d} S^{\prime}\right) .
$$

We claim that the vanishing locus of $\left\langle\alpha, V_{a}\right\rangle$ in this case coincides with the critical surfaces of the original Yang-Mills action (2.25) as found in Section 3.1. To see this, we note that the condition

$$
0=\left\langle\alpha, V_{a}\right\rangle=\operatorname{Tr}\left(C_{0}\left[C,\left[C, \phi^{a}\right]\right]_{0}\right)=-\operatorname{Tr}\left(\left[C, C_{0}\right]\left[C, \phi^{a}\right]\right)
$$


certainly holds whenever $\left[C, C_{0}\right]=0$. On the other hand, by setting $\phi=C_{0}$ it implies

$$
0=\left\langle\alpha, V_{\phi}\right\rangle=-\operatorname{Tr}\left(\left[C, C_{0}\right]^{2}\right)
$$

which by nondegeneracy of the inner product implies that $\left[C, C_{0}\right]=0$. Therefore the action in (4.10) has indeed the same critical points as the Yang-Mills action (2.25).

Let us now explicitly establish, following [32], the localization of the partition function onto the classical solutions of the gauge theory. Plugging (4.14) and (4.11) into (4.10) and carrying out the integration over $\phi \in \mathfrak{g}$ gives

$$
\begin{aligned}
Z=\frac{1}{\operatorname{vol}(G)} \int_{\mathfrak{g} \times \mathcal{O}} & {\left[\frac{\mathrm{d} \phi}{2 \pi}\right] \exp (t \mathrm{~d} \alpha+\omega) } \\
& \times \exp \left(-\mathrm{i} \operatorname{Tr}\left(C_{0} \phi\right)-\frac{g^{\prime}}{2} \operatorname{Tr}\left(\phi^{2}\right)-\mathrm{i} t \operatorname{Tr}\left(\left[C,\left[C, C_{0}\right]\right] \phi\right)\right) \\
= & \frac{1}{\operatorname{vol}(G)}\left(\frac{g^{\prime}}{2 \pi}\right)^{\operatorname{dim}(G) / 2} \int_{\mathcal{O}} \exp (t \mathrm{~d} \alpha+\omega) \\
& \times \exp \left(-\frac{1}{2 g^{\prime}} \operatorname{Tr}\left(C_{0}^{2}\right)+\frac{t}{g^{\prime}} \operatorname{Tr}\left(C_{0}\left[C,\left[C, C_{0}\right]\right]\right)-\frac{t^{2}}{2 g^{\prime}} \operatorname{Tr}\left(\left[C,\left[C, C_{0}\right]\right]\right)^{2}\right)
\end{aligned}
$$

where we have used $\operatorname{Tr}(C[C,-])=0$. The only configurations which contribute to (4.17) in the large $t$ limit are therefore solutions of the equation

$$
\left[C,\left[C, C_{0}\right]\right]=0
$$

which implies as in [32] that

$$
0=\operatorname{Tr}\left(C_{0}\left[C,\left[C, C_{0}\right]\right]\right)=-\operatorname{Tr}\left(\left[C, C_{0}\right]^{2}\right),
$$

giving $\left[C, C_{0}\right]=0$ as desired. Therefore the integral (4.17) receives contributions only from the solutions of the Yang-Mills equations (3.2), which establishes the claimed localization.

The local geometry in $\mathfrak{g} \times \mathcal{O}$ about each critical point, as analysed in detail in the last section, determines the partition function as a sum of local contributions involving the values of the YangMills action evaluated on the classical solutions as in Section 3.2. Consider an equivariant tubular neighbourhood $\mathcal{N}_{\left(n_{1}, s_{1}\right), \ldots,\left(n_{k}, s_{k}\right)}$ of a critical surface $\mathcal{C}_{\left(n_{1}, s_{1}\right), \ldots,\left(n_{k}, s_{k}\right)}$ in $\mathfrak{g} \times \mathcal{O}$. Since the partition function (4.10) is independent of $t$, we can consider its large $t$ limit as above, and this limit will always be implicitly assumed from now on. Let $\mathcal{W}$ be a compact subset of $\mathcal{O}$ with $\mathcal{W} \cap \mathcal{C}=\emptyset$, where $\mathcal{C}:=\bigcup_{\left(n_{i}, s_{i}\right)} \mathcal{C}_{\left(n_{1}, s_{1}\right), \ldots,\left(n_{k}, s_{k}\right)}$. Then the integral over $\mathcal{W}$ in (4.17) has a gaussian decay in $t \rightarrow \infty$. This means that in expanding $\exp (t \mathrm{~d} \alpha+\omega)$ into a finite sum of terms of the form $\omega^{p} \wedge(t \mathrm{~d} \alpha)^{m}$, we can disregard all terms which contain $\omega$ since they will be suppressed by factors of $\frac{1}{t}$ and vanish in the large $t$ limit. The only terms which survive the $t \rightarrow \infty$ limit are those with $p=0, m=d$, and the integral therefore vanishes unless $\omega$ is replaced by $\mathrm{d} \alpha$, except at the saddle point where $\mathrm{d} \alpha=0$. Then one has

$$
Z=\frac{1}{\operatorname{vol}(G)} \int_{\mathfrak{g} \times \mathcal{O}}\left[\frac{\mathrm{d} \phi}{2 \pi}\right] \exp \left(t\left(\mathrm{~d} \alpha-\mathrm{i}\left\langle\alpha, V_{\phi}\right\rangle\right)\right) \exp \left(-\mathrm{i} \operatorname{Tr}\left(C_{0} \phi\right)-\frac{g^{\prime}}{2} \operatorname{Tr}\left(\phi^{2}\right)\right)
$$

in the vicinity of any critical point in which $\mathrm{d} \alpha$ is nondegenerate.

The integral $Z_{\left(n_{1}, s_{1}\right), \ldots,\left(n_{k}, s_{k}\right)}$ in (4.20) over the neighbourhood $\mathcal{N}_{\left(n_{1}, s_{1}\right), \ldots,\left(n_{k}, s_{k}\right)}$ is determined by the local behaviour of $\alpha$ and the $G$-action near $\mathcal{C}_{\left(n_{1}, s_{1}\right), \ldots,\left(n_{k}, s_{k}\right)}$. Then

$$
Z=\sum_{\substack{\left(n_{1}, s_{1}\right), \ldots,\left(n_{k}, s_{k}\right) \\ \sum_{i} n_{i}=n N, \sum_{i} s_{i}=n}} Z_{\left(n_{1}, s_{1}\right), \ldots,\left(n_{k}, s_{k}\right)} .
$$


As expected [24], the sum over critical surfaces in (4.21) contains the sum over weights $1 \leq n_{1} \leq$ $n_{2} \leq \cdots \leq n_{k}$ of the gauge group $G=U(n N)$. Our explicit computations will confirm the local behaviour of the partition function given by [24]

$$
Z_{\left(n_{1}, s_{1}\right), \ldots,\left(n_{k}, s_{k}\right)}=\left(g^{\prime}\right)^{-\operatorname{dim}(G)} \mathrm{e}^{-\frac{1}{2 g^{\prime}} \sum_{i} n_{i}^{2}} H_{\left(n_{1}, s_{1}\right), \ldots,\left(n_{k}, s_{k}\right)}\left(\sqrt{g^{\prime}}\right) .
$$

The smooth function $H_{\left(n_{1}, s_{1}\right), \ldots,\left(n_{k}, s_{k}\right)}: \mathbb{R} \rightarrow \mathbb{C}$, which is bounded by a polynomial at infinity, is determined by the equivariant Euler class of the fixed point locus corresponding to the weight $\left(n_{1}, \ldots, n_{k}\right)$ after reducing the integral over $\mathfrak{g}$ to its Cartan subalgebra, as we do explicitly in the next section.

\subsection{Explicit evaluation of the localization forms}

The explicit computation of the local contributions $Z_{\left(n_{1}, s_{1}\right), \ldots,\left(n_{k}, s_{k}\right)}$ to the Yang-Mills partition function on $S_{N}^{2}$ will rely on the local behaviour of the invariant one-form $\alpha$ introduced in (4.14) near the Yang-Mills critical points. We will now pause to derive explicit expressions for the BRST transformations (4.11) on the subspaces appearing in the tangent space decomposition (3.36). Given the invariant Maurer-Cartan one-form (2.42) and the projector (3.63), consider the $\mathfrak{u}(n N)$-valued one-form

$$
\theta_{0}:=\Pi_{0}(\theta)=\frac{1}{2} \operatorname{tr}_{\sigma}(\theta)
$$

where $\operatorname{tr}_{\sigma}$ denotes the partial trace over the spin matrices $\sigma^{\mu}$. It is given explicitly by

$$
\theta_{0}=\frac{4}{N^{2}}(C \mathrm{~d} C)_{0}=\frac{4}{N^{2}}\left(C_{i} \mathrm{~d} C^{i}+C_{0} \mathrm{~d} C_{0}\right)
$$

and satisfies

$$
\mathrm{d} \theta_{0}=-\frac{1}{2} \operatorname{tr}_{\sigma}\left(\theta^{2}\right)=-\Pi_{0}\left(\theta^{2}\right)
$$

One has

$$
\left\langle\theta, V_{\phi}\right\rangle=\frac{2}{N^{2}}\left[C, V_{\phi}\right]=-\frac{2 \mathrm{i}}{N} \mathcal{J}\left(V_{\phi}\right) \quad \text { and } \quad\left\langle\theta_{0}, V_{\phi}\right\rangle=-\frac{2 \mathrm{i}}{N} \Pi_{0} \mathcal{J}\left(V_{\phi}\right)
$$

for any tangent vector $V_{\phi}=\mathrm{i}[C, \phi]$.

Using the identity $C \mathrm{~d} C=-\mathrm{d} C C$, the localization one-form (4.14) can now be written as

$$
\alpha=-\frac{\mathrm{i} N^{2}}{2} \operatorname{Tr}\left(C_{0} \theta\right)=-\frac{\mathrm{i} N^{2}}{2} \operatorname{Tr}\left(C \theta_{0}\right) .
$$

Hence the pairing in (4.11) is given by

$$
\begin{aligned}
\left\langle\alpha, V_{\phi}\right\rangle & =-N \operatorname{Tr}\left(C_{0} \mathcal{J}\left(V_{\phi}\right)\right) \\
& =N \operatorname{Tr}\left(\mathcal{J}\left(C_{0}\right) V_{\phi}\right)=-N^{2} \operatorname{Tr}\left(\mathcal{J}^{2}\left(C_{0}\right) \phi\right) .
\end{aligned}
$$

This vanishes on the critical surfaces, where $\mathcal{J}\left(C_{0}\right)=0$. Furthermore, for any $g \in \mathfrak{g}$ one has

$$
\begin{aligned}
\left\langle\alpha, \mathcal{J}^{2}(g)\right\rangle & =-N \operatorname{Tr}\left(C_{0} \mathcal{J}^{3}(g)\right) \\
& =N \operatorname{Tr}\left(C_{0} \mathcal{J}(g)\right)=\mathrm{i} \operatorname{Tr}\left(C_{0}\left[C_{0}, g\right]\right)=0
\end{aligned}
$$

while for $e_{0} \in E_{0}$ one has

$$
\left\langle\alpha, e_{0}\right\rangle=\left\langle\alpha, \mathcal{J}\left(e_{0}\right)\right\rangle=\left\langle\alpha, \mathcal{J}^{2}(h)\right\rangle=0
$$

for some $h \in \mathfrak{h}$. Both identities (4.29) and (4.30) hold even off-shell. We also note the on-shell relations

$$
\langle\alpha, \mathcal{J}(g)\rangle=-N \operatorname{Tr}\left(C_{0} \mathcal{J}^{2}(g)\right)=0 \quad \text { and } \quad\left\langle\alpha, e_{1}\right\rangle=-N \operatorname{Tr}\left(C_{0} \mathcal{J}\left(e_{1}\right)\right)=0
$$


for $e_{1} \in E_{1}$.

To evaluate the integral (4.20) using the stationary phase method, we must understand how it behaves near the Yang-Mills critical points. For this, we will study the local behaviour of the BRST variation (4.11), beginning with the pairing $\left\langle\alpha, V_{\phi}\right\rangle$. Let us write a generic gauge field of $\mathcal{O}$ as $C=\bar{C}+\varepsilon \mathrm{i}[\bar{C}, \Psi]+\frac{1}{2} \varepsilon^{2} \mathrm{i}[\bar{C}, \mathrm{i}[\bar{C}, \Psi]]+O\left(\varepsilon^{3}\right)$, where $\bar{C}$ is the given critical point, $\Psi \in \mathfrak{s u}(\mathcal{N})$ are the fluctuations around $\bar{C}$ and $\varepsilon$ is a small real parameter. Then

$$
\begin{aligned}
\mathcal{J}^{2}\left(C_{0}\right) & =0+\varepsilon\left(\mathcal{J}^{2}\left(\mathrm{i}[\bar{C}, \Psi]_{0}\right)+\frac{\mathrm{i}}{N}\left[\mathrm{i}[\bar{C}, \Psi], \mathcal{J}\left(\bar{C}_{0}\right)\right]+\frac{\mathrm{i}}{N} \mathcal{J}\left(\left[\mathrm{i}[\bar{C}, \Psi], \bar{C}_{0}\right]\right)\right)+O\left(\varepsilon^{2}\right) \\
& =\varepsilon\left(\mathcal{J}^{2}\left(\left(V_{\Psi}\right)_{0}\right)+\frac{\mathrm{i}}{N} \mathcal{J}\left(\left[V_{\Psi}, \bar{C}_{0}\right]\right)\right)+O\left(\varepsilon^{2}\right)
\end{aligned}
$$

which for $\phi \in \mathfrak{g}$ gives

$$
\begin{aligned}
\left\langle\alpha, V_{\phi}\right\rangle & =-\varepsilon N^{2} \operatorname{Tr}\left(\mathcal{J}^{2}\left(\left(V_{\Psi}\right)_{0}\right) \phi+\frac{\mathrm{i}}{N}\left[\mathcal{J}\left(V_{\Psi}\right), \bar{C}_{0}\right], \phi\right)+O\left(\varepsilon^{2}\right) \\
& =-\varepsilon N^{2} \operatorname{Tr}\left(\left(V_{\Psi}\right)_{0} \mathcal{J}^{2}(\phi)+\mathcal{J}\left(V_{\Psi}\right)\left[\frac{\mathrm{i}}{N} \bar{C}_{0}, \phi\right]\right)+O\left(\varepsilon^{2}\right) \\
& =-\varepsilon N^{2} \operatorname{Tr}\left(V_{\Psi}\left(\mathcal{J}^{2}(\phi)_{0}-\mathcal{J}\left(\mathcal{J}(\phi)_{0}\right)\right)\right)+O\left(\varepsilon^{2}\right) .
\end{aligned}
$$

using (3.65). This is non-degenerate for $\phi \in \mathfrak{g} \ominus \mathfrak{s} \ominus \mathfrak{h}$, i.e. non-vanishing for some $V_{\Psi} \in T_{C} \mathcal{O}$. To see this, it is sufficient to show that $\mathcal{J}\left(\mathcal{J}^{2}(\phi)_{0}-\mathcal{J}\left(\mathcal{J}(\phi)_{0}\right)\right) \neq 0$. Indeed, assuming the contrary $\mathcal{J}\left(\mathcal{J}^{2}(\phi)_{0}\right)=\mathcal{J}^{2}\left(\mathcal{J}(\phi)_{0}\right)$ would imply that either $\phi \in \mathfrak{s}$, or $\mathcal{J}(\phi)_{0} \in \mathfrak{h}$ which is amounts to $\phi \in \mathfrak{h} \oplus \mathfrak{s}$. On the other hand, this pairing is indeed degenerate for any $V_{\Psi} \in E_{1}$.

For $\phi \in \mathfrak{s}$, the second-order contribution to the form (4.33) can be obtained from

$$
V_{\phi}=\mathrm{i}[C, \phi]=\mathrm{i} \varepsilon\left[V_{\Psi}, \phi\right]+O\left(\varepsilon^{2}\right)
$$

and

$$
\mathcal{J}\left(C_{0}\right)=\frac{\mathrm{i}}{N}\left[C, C_{0}\right]=\frac{\mathrm{i}}{N} \varepsilon\left(\left[V_{\Psi}, C_{0}\right]+\left[C,\left(V_{\Psi}\right)_{0}\right]\right)+O\left(\varepsilon^{2}\right)
$$

It follows that

$$
\left\langle\alpha, V_{\phi}\right\rangle=-\varepsilon^{2} \operatorname{Tr}\left(\operatorname{ad}_{\phi}\left(V_{\Psi}\right)\left(\operatorname{ad}_{C_{0}}\left(V_{\Psi}\right)+\mathrm{i} N \mathcal{J}\left(\left(V_{\Psi}\right)_{0}\right)\right)\right)+O\left(\varepsilon^{3}\right) .
$$

In particular, for $V_{\Psi} \in E_{1}$ this pairing simplifies to

$$
\left\langle\alpha, V_{\phi}\right\rangle=-\varepsilon^{2} \operatorname{Tr}\left(\operatorname{ad}_{\phi}\left(V_{\Psi}\right) \operatorname{ad}_{C_{0}}\left(V_{\Psi}\right)\right)+O\left(\varepsilon^{3}\right) .
$$

We now turn to the exact part $\mathrm{d} \alpha$ of (4.11). Using (2.44)-(2.46), one finds

$$
\mathrm{d} \alpha=-\mathrm{i} \frac{N^{2}}{2} \operatorname{Tr}\left(\mathrm{d} C \theta_{0}-C_{0} \theta^{2}\right)=-\mathrm{i} \frac{N^{2}}{2} \operatorname{Tr}\left(C \theta \theta_{0}+C_{0} \mathrm{~d} \theta\right) .
$$

For flat connections with $F=0$, the second term in the first equality of (4.38) vanishes and one has

$$
\mathrm{d} \alpha=-\mathrm{i} \frac{N^{2}}{2} \operatorname{Tr}\left(\mathrm{d} C \theta_{0}\right)=-\mathrm{i} \frac{N^{2}}{2} \operatorname{Tr}\left(C \theta \theta_{0}\right) \quad \text { if } \quad C_{0}=\frac{1}{2} \mathbb{1}_{n N} .
$$

From (2.43) and (2.44) one generally has $\theta^{2}=-\frac{4}{N^{2}}(\mathrm{~d} C)^{2}$, and hence

$$
\begin{aligned}
\left\langle\operatorname{Tr}\left(C_{0} \theta^{2}\right), V_{\phi} \wedge V_{\psi}\right\rangle & =\frac{4}{N^{2}} \operatorname{Tr}\left(C_{0}[[C, \phi],[C, \psi]]\right) \\
& =\frac{4}{N^{2}} \operatorname{Tr}\left(\left[C_{0},[C, \phi]\right][C, \psi]\right)=-\frac{4}{N^{2}} \operatorname{Tr}\left(\operatorname{ad}_{C_{0}}\left(V_{\phi}\right) V_{\psi}\right)
\end{aligned}
$$

for any pair of tangent vectors $V_{\phi}=\mathrm{i}[C, \phi]$ and $V_{\psi}=\mathrm{i}[C, \psi]$. Similarly, one has

$$
\left\langle\operatorname{Tr}\left(C \theta \theta_{0}\right), V_{\phi} \wedge V_{\psi}\right\rangle=\left\langle\operatorname{Tr}\left(\mathrm{d} C \theta_{0}\right), V_{\phi} \wedge V_{\psi}\right\rangle=-\frac{2 \mathrm{i}}{N} \operatorname{Tr}\left(V_{\phi} \mathcal{J}\left(V_{\psi}\right)_{0}-V_{\psi} \mathcal{J}\left(V_{\phi}\right)_{0}\right)
$$


which vanishes if any of the arguments belongs to the subspace $E_{1}$.

If $V_{\psi}=\mathcal{J}(h) \in E_{0}$ for some $h \in \mathfrak{h}$, then by using the map (3.35) along with (4.41) one computes the on-shell pairing

$$
\begin{aligned}
\left\langle\operatorname{Tr}\left(C \theta \theta_{0}\right), V_{\phi} \wedge V_{\psi}\right\rangle & =-\frac{2 \mathrm{i}}{N} \operatorname{Tr}\left(V_{\phi} \mathcal{J}(j(h))_{0}-\mathcal{J}(h)_{0} \mathcal{J}\left(V_{\phi}\right)\right) \\
& =-\frac{2}{N^{2}} \operatorname{Tr}\left(\operatorname{ad}_{C_{0}}\left(V_{\phi}\right) j(h)+\operatorname{ad}_{C_{0}}(h) \mathcal{J}\left(V_{\phi}\right)\right) \\
& =-\frac{2}{N^{2}} \operatorname{Tr}\left(N \operatorname{ad}_{C_{0}}(\mathcal{J}(\phi)) j(h)+\operatorname{ad}_{C_{0}}(h) \mathcal{J}\left(V_{\phi}\right)\right) \\
& =-\frac{2}{N^{2}} \operatorname{Tr}\left(-N \operatorname{ad}_{C_{0}}(\phi) \mathcal{J}^{2}(h)+\operatorname{ad}_{C_{0}}(h) \mathcal{J}\left(V_{\phi}\right)\right) \\
& =-\frac{2}{N^{2}} \operatorname{Tr}\left(N \operatorname{ad}_{C_{0}}(\mathcal{J}(\phi)) \mathcal{J}(h)-\operatorname{ad}_{C_{0}}(\mathcal{J}(h)) V_{\phi}\right) \\
& =-\frac{2}{N^{2}} \operatorname{Tr}\left(\operatorname{ad}_{C_{0}}\left(V_{\phi}\right) V_{\psi}-\operatorname{ad}_{C_{0}}\left(V_{\psi}\right) V_{\phi}\right) .
\end{aligned}
$$

This coincides with (4.40), and in particular it vanishes unless the vector field $V_{\phi}$ also belongs to the subspace $E_{0}$. In summary, we have the on-shell evaluations

$$
\left\langle\mathrm{d} \alpha, V_{\phi} \wedge V_{\psi}\right\rangle=2 \mathrm{i} \operatorname{Tr}\left(V_{\phi} \operatorname{ad}_{C_{0}}\left(V_{\psi}\right)\right) \quad \text { if } \quad V_{\psi} \in E_{1}
$$

and

$$
\left\langle\mathrm{d} \alpha, V_{\phi} \wedge V_{\psi}\right\rangle=0 \quad \text { if } \quad V_{\psi} \in E_{0} .
$$

\subsection{Localization at the vacuum moduli space}

We will now compute the localized partition function $Z_{0}:=Z_{(N, 1), \ldots,(N, 1)}$ at the vacuum critical surface. We denote this gauge orbit as

$$
\mathcal{O}_{0}:=\mathcal{C}_{(N, 1), \ldots,(N, 1)}=\left\{g C g^{-1} \mid g \in U(n N)\right\} \cong U(n N) / U(n)
$$

In this case the subspaces $E_{0}$ and $E_{1}$ in (3.36) are trivial. Localization implies that we can restrict ourselves to a $G$-equivariant tubular neighbourhood $\mathcal{N}_{0}=\mathcal{N}_{(N, 1), \ldots,(N, 1)}$ of the critical surface, under the action of the gauge group $G=U(n N)$. The neighbourhood $\mathcal{N}_{0}$ has an equivariant retraction [45, Chap. 27] by a local equivariant symplectomorphism onto the local symplectic model $\mathcal{F}_{0}$, defined to be an equivariant symplectic vector bundle over $\mathcal{O}_{0}$ with fibre $\mathcal{J}^{2}(\mathfrak{g} \ominus \mathfrak{s})$ which is a sub-bundle of the tangent bundle $T \mathcal{O}$ restricted to $\mathcal{O}_{0}$. This means that the tangent space to $\mathcal{F}_{0}$ at the vacuum critical point $C$ in (3.9) is given by $T_{C} \mathcal{O}_{0} \oplus \mathcal{J}^{2}(\mathfrak{g} \ominus \mathfrak{s}) \cong \mathcal{J}(\mathfrak{g} \ominus \mathfrak{s}) \oplus \mathcal{J}^{2}(\mathfrak{g} \ominus \mathfrak{s})=T_{C} \mathcal{O}$, the symplectic two-form on $\mathcal{F}_{0}$ is simply $\omega$, and the hamiltonian $G$-action on $\mathcal{F}_{0}$ descends from the moment map $\mu$. In physical terms, the gauge fields are decomposed along the vacuum moduli space $\mathcal{O}_{0}$ plus infinitesimal non-gauge variations in the subspace $\mathcal{J}^{2}(\mathfrak{g} \ominus \mathfrak{s})$.

Due to the presence of the localization form $\alpha$ in the path integral, we can restrict ourselves to this model $\mathcal{F}_{0}$ and use it to replace the open neighbourhood $\mathcal{N}_{0}$ [27]. Indeed, because $\mathcal{F}_{0}$ is an equivariant retraction from $\mathcal{N}_{0}$, the $G$-equivariant cohomology of $\mathcal{N}_{0}$ is the same as that of $\mathcal{F}_{0}$. Furthermore, since the fibres of the bundle $\mathcal{F}_{0}$ are contractible, its $G$-equivariant cohomology is identified under pullback with the $S$-equivariant cohomology of its base space $\mathcal{O}_{0}$, so that $H_{G}\left(\mathcal{N}_{0}\right) \cong$ $H_{S}\left(\mathcal{O}_{0}\right)$. Since $S$ acts trivially on $\mathcal{O}_{0}$, one has $H_{S}\left(\mathcal{O}_{0}\right) \cong \mathbb{C}[[\mathfrak{s}]]^{S} \otimes H\left(\mathcal{O}_{0}\right)$ and the $S$-equivariant cohomology classes of $\mathcal{O}_{0}$ coincide with ordinary cohomology classes of $\mathcal{O}_{0}$ valued in the ring of invariant functions on the stabilizer $\mathfrak{s}$. Putting everything together gives an isomorphism $H_{G}\left(\mathcal{N}_{0}\right) \cong$ $\mathbb{C}[[\mathfrak{s}]]^{S} \otimes H\left(\mathcal{O}_{0}\right)$ which reduces the equivariant integral over $\mathfrak{g} \times \mathcal{N}_{0}$ in (4.20) to an ordinary integral over $\mathfrak{s} \times \mathcal{O}_{0}$. This is precisely the nonabelian localization that is formally carried out in [26], and will turn out to be very much like the localization at the trivial connection of Chern-Simons theory on a Seifert homology sphere [27]. In the present case, the integral over $\phi \in \mathfrak{s}$ will then give the 
interesting non-trivial quantum fluctuation determinants about the classical solution. We will now carry out this reduction explicitly.

Let $g_{i}^{\prime}$ be an orthonormal basis of $\mathfrak{g}^{\prime}=\mathfrak{g} \ominus \mathfrak{s}$, and consider the corresponding basis

$$
J_{i}=\mathcal{J}\left(g_{i}^{\prime}\right) \quad \text { and } \quad \tilde{J}_{j}=\mathcal{J}^{2}\left(g_{j}^{\prime}\right)
$$

of $T_{C} \mathcal{O}=\mathcal{J}(\mathfrak{g} \ominus \mathfrak{s}) \oplus \mathcal{J}^{2}(\mathfrak{g} \ominus \mathfrak{s})$, with the dual basis $\lambda^{i}, \tilde{\lambda}^{j}$ defined by

$$
\left\langle\lambda^{i}, J_{j}\right\rangle=\delta^{i}{ }_{j}, \quad\left\langle\tilde{\lambda}^{i}, \tilde{J}_{j}\right\rangle=\delta^{i}{ }_{j} \quad \text { and } \quad\left\langle\lambda^{i}, \tilde{J}_{j}\right\rangle=\left\langle\tilde{\lambda}^{i}, J_{j}\right\rangle=0 .
$$

Introduce the functions

$$
f_{i}=\left\langle\alpha, J_{i}\right\rangle
$$

which vanish on-shell but have non-degenerate derivatives $\mathrm{d} f_{i}$ due to (4.33). Then by expanding $\phi=\phi^{i} g_{i}+\phi^{a} s_{a}$ into components $\phi^{i}$ along $\mathfrak{g} \ominus \mathfrak{s}$ and $\phi^{a}$ along $\mathfrak{s}$, we have

$$
\left\langle\alpha, V_{\phi}\right\rangle=N\langle\alpha, \mathcal{J}(\phi)\rangle=N f_{i} \phi^{i}
$$

It follows that the localization one-form can be expanded as

$$
\alpha=f_{i} \lambda^{i}
$$

with

$$
\mathrm{d} \alpha=\mathrm{d} f_{i} \wedge \lambda^{i}+f_{i} \mathrm{~d} \lambda^{i}
$$

In particular, one has

$$
\frac{(\mathrm{d} \alpha)^{d}}{d !}=\bigwedge_{i=1}^{d}\left(\mathrm{~d} f_{i} \wedge \lambda^{i}\right)+f_{j} \Upsilon^{j}
$$

where $d=\operatorname{dim}_{\mathbb{C}}(\mathcal{O})=n^{2}\left(N^{2}-1\right)$ is the (real) dimension of the vacuum orbit $\mathcal{O}_{0}$. The forms $f_{j} \Upsilon^{j}$ vanish on-shell, and are killed by localization in the large $t$ limit. For example, inner products of the form $\langle\alpha, \mathcal{J}(s)\rangle, s \in \mathfrak{s}$ are non-vanishing off-shell at second order due to (4.36), but these higher-order terms do not contribute because of the localization in the large $t$ limit. This can be seen explicitly by rescaling $f_{i}=\sqrt{t} f_{i}^{\prime}$.

The corresponding local contribution to the partition function (4.20) for $t \rightarrow \infty$ is then given by

$$
\begin{aligned}
Z_{0} & =\frac{1}{\operatorname{vol}(G)} \int_{\mathfrak{g} \times \mathcal{F}_{0}}\left[\frac{\mathrm{d} \phi}{2 \pi}\right] \frac{t^{d}}{d !}(\mathrm{d} \alpha)^{d} \mathrm{e}^{-\mathrm{i} t\left\langle\alpha, V_{\phi}\right\rangle-\mathrm{i} \operatorname{Tr}\left(C_{0} \phi\right)-\frac{g^{\prime}}{2} \operatorname{Tr}\left(\phi^{2}\right)} \\
& =\frac{1}{\operatorname{vol}(G)} \int_{\mathfrak{g} \times \mathcal{F}_{0}}\left[\frac{\mathrm{d} \phi}{2 \pi}\right] t^{d} \bigwedge_{i=1}^{d}\left(\mathrm{~d} f_{i} \wedge \lambda^{i}\right) \mathrm{e}^{-\mathrm{i} N t f_{i} \phi^{i}-\mathrm{i} \operatorname{Tr}\left(C_{0} \phi\right)-\frac{g^{\prime}}{2} \operatorname{Tr}\left(\phi^{2}\right)} \\
& =\frac{1}{\operatorname{vol}(G)} \int_{\mathfrak{s}}\left[\frac{\mathrm{d} \phi}{2 \pi}\right] \mathrm{e}^{-\mathrm{i} \operatorname{Tr}\left(C_{0} \phi\right)-\frac{g^{\prime}}{2} \operatorname{Tr}\left(\phi^{2}\right)} \frac{1}{N^{d}} \int_{\mathcal{O}_{0}} \bigwedge_{i=1}^{d} \lambda^{i} .
\end{aligned}
$$

Here the $f_{i}$ integrals over the fibre $\mathcal{J}^{2}(\mathfrak{g} \ominus \mathfrak{s})$ have produced delta-functions setting $\phi^{i}=0$ in $\mathfrak{g} \ominus \mathfrak{s}$. We can carry out the integral over the moduli space $\mathcal{O}_{0}$ in (4.53) by observing that

$$
\frac{1}{N^{d}} \int_{\mathcal{O}_{0}} \bigwedge_{i=1}^{d} \lambda^{i}=\int_{G / S} \bigwedge_{i=1}^{d} \eta^{i}=\frac{\operatorname{vol}(G)}{\operatorname{vol}(S)}
$$


where the pullbacks $\mathcal{J}^{*}\left(\lambda^{i}\right)=\eta^{i}$ define left-invariant one-forms on the gauge group $G$ dual to $g_{i}^{\prime}$, with the map $N \mathcal{J}$ regarded as the derivative of the diffeomorphism

$$
G / S \longrightarrow \mathcal{O}_{0}, \quad g \longmapsto g C g^{-1}
$$

To evaluate the remaining integral over the gauge stabilizer algebra $\mathfrak{s} \cong \mathfrak{u}(n)$ in (4.53), we note that, for the vacuum critical point with $C_{0}=\frac{1}{2} \mathbb{1}_{n N}$, the integrand defines a gauge invariant function $f: \mathfrak{u}(n) \rightarrow \mathbb{R}$. We may thus apply to it the Weyl integration formula which reduces its integral over $\mathfrak{u}(n)$ to an integral over the Lie algebra $\mathfrak{u}(1)^{n}$ of the maximal torus $U(1)^{n}$ of $U(n)$. It is given by

$$
\int_{\mathfrak{u}(n)}[\mathrm{d} \phi] f(\phi)=\frac{\operatorname{vol}(U(n))}{n !(2 \pi)^{n}} \int_{\mathbb{R}^{n}}[\mathrm{~d} s] \Delta(s)^{2} f(s),
$$

where we have identified $\mathfrak{u}(1)^{n} \cong \mathbb{R}^{n}$ in a basis where the Cartan subalgebra of $U(n)$ is represented by diagonal $n \times n$ matrices $s=\operatorname{diag}\left(s_{1}, \ldots, s_{n}\right)$ by mapping them onto $n$-vectors $s=\left(s_{1}, \ldots, s_{n}\right) \in$ $\mathbb{R}^{n}$. Here

$$
\Delta(s)=\prod_{i<j}\left(s_{i}-s_{j}\right)=\operatorname{det}_{1 \leq i, j \leq n}\left(s_{i}^{j-1}\right)
$$

is the Vandermonde determinant, which is the Weyl determinant for $U(n)$ arising as the jacobian for the diagonalization of hermitian matrices on the left-hand side of (4.56). The factor $n$ ! is the order of the Weyl group $\mathfrak{S}_{n}$ of $U(n)$ acting by permutations of the components $s_{i}$ of $s \in \mathbb{R}^{n}$, while $(2 \pi)^{n}$ is the volume of the maximal torus $U(1)^{n}$ with respect to the chosen invariant Haar measure.

An integral identity

We will make use here and in Section 4.4 below of the integral identity

$$
\begin{aligned}
\int_{\mathbb{R}^{n}}[\mathrm{~d} s] \Delta(s)^{2} & \mathrm{e}^{-\mathrm{i} \frac{N}{2} \sum_{i} s_{i}+\frac{\mathrm{i}}{4} \sum_{i} m_{i} s_{i}-\frac{g}{4} \sum_{i} s_{i}^{2}} \\
& =\mathrm{e}^{-\frac{n N^{2}-m N}{4 g}} \int_{\mathbb{R}^{n}}[\mathrm{~d} s] \Delta(s)^{2} \mathrm{e}^{\frac{\mathrm{i}}{4} \sum_{i} m_{i} s_{i}-\frac{g}{4} \sum_{i} s_{i}^{2}}
\end{aligned}
$$

where $m=\sum_{i} m_{i}$. To derive (4.58), we set $s=\sum_{i} s_{i}$ and $t_{i}=s_{i}-\frac{1}{n} s$ so that $\sum_{i} t_{i}=0$. Then

$$
\begin{aligned}
\int_{\mathbb{R}^{n}}[\mathrm{~d} s] \Delta(s)^{2} & \mathrm{e}^{-\mathrm{i} \frac{N}{2} \sum_{i} s_{i}+\frac{\mathrm{i}}{4} \sum_{i} m_{i} s_{i}-\frac{g}{4} \sum_{i} s_{i}^{2}} \\
& =\int_{\mathbb{R}} \mathrm{d} s \mathrm{e}^{-\mathrm{i} \frac{N}{2} s+\mathrm{i} \frac{m}{4 n} s} \int_{\mathbb{R}^{n}}[\mathrm{~d} t] \Delta(t)^{2} \mathrm{e}^{\frac{\mathrm{i}}{4} \sum_{i} m_{i} t_{i}-\frac{g}{4} \sum_{i}\left(t_{i}+\frac{1}{n} s\right)^{2}} \\
& =\int_{\mathbb{R}} \mathrm{d} s \mathrm{e}^{-\mathrm{i} \frac{N}{2} s+\mathrm{i} \frac{m}{4 n} s-\frac{g}{4 n} s^{2}} \int_{\mathbb{R}^{n}}[\mathrm{~d} t] \Delta(t)^{2} \mathrm{e}^{\frac{\mathrm{i}}{4} \sum_{i} m_{i} t_{i}-\frac{g}{4} \sum_{i} t_{i}^{2}} \\
& =2 \sqrt{\frac{\pi n}{g}} \mathrm{e}^{-\frac{(2 n N-m)^{2}}{16 n g}} \int_{\mathbb{R}^{n}}[\mathrm{~d} t] \Delta(t)^{2} \mathrm{e}^{\frac{\mathrm{i}}{4} \sum_{i} m_{i} t_{i}-\frac{g}{4} \sum_{i} t_{i}^{2}} .
\end{aligned}
$$

On the other hand

$$
\begin{aligned}
\int_{\mathbb{R}^{n}}[\mathrm{~d} s] \Delta(s)^{2} \mathrm{e}^{\frac{\mathrm{i}}{4} \sum_{i} m_{i} s_{i}-\frac{g}{4} \sum_{i} s_{i}^{2}} & =\int_{\mathbb{R}} \mathrm{d} s \mathrm{e}^{\mathrm{i} \frac{m}{4 n} s} \int_{\mathbb{R}^{n}}[\mathrm{~d} t] \Delta(t)^{2} \mathrm{e}^{\frac{\mathrm{i}}{4} \sum_{i} m_{i} t_{i}-\frac{g}{4} \sum_{i}\left(t_{i}+\frac{1}{n} s\right)^{2}} \\
& =\int_{\mathbb{R}} \mathrm{d} s \mathrm{e}^{\mathrm{i} \frac{m}{4 n} s-\frac{g}{4 n} s^{2}} \int_{\mathbb{R}^{n}}[\mathrm{~d} t] \Delta(t)^{2} \mathrm{e}^{\frac{\mathrm{i}}{4} \sum_{i} m_{i} t_{i}-\frac{g}{4} \sum_{i} t_{i}^{2}} \\
& =2 \sqrt{\frac{\pi n}{g}} \mathrm{e}^{-\frac{m^{2}}{16 n g}} \int_{\mathbb{R}^{n}}[\mathrm{~d} t] \Delta(t)^{2} \mathrm{e}^{\frac{\mathrm{i}}{4} \sum_{i} m_{i} t_{i}-\frac{g}{4} \sum_{i} t_{i}^{2}} .
\end{aligned}
$$


Final reduction

From (4.53), (4.54) and (4.56) we obtain

$$
\begin{aligned}
Z_{0} & =\frac{1}{\operatorname{vol}(S)} \int_{\mathfrak{s}}\left[\frac{\mathrm{d} \phi}{2 \pi}\right] \mathrm{e}^{-\mathrm{i} \operatorname{Tr}\left(C_{0} \phi\right)-\frac{g^{\prime}}{2} \operatorname{Tr}\left(\phi^{2}\right)} \\
& =\frac{1}{n !} \frac{1}{(2 \pi)^{n^{2}}} \int_{\mathbb{R}^{n}}\left[\frac{\mathrm{d} s}{2 \pi}\right] \Delta(s)^{2} \mathrm{e}^{-\mathrm{i} \frac{N}{2} \sum_{i} s_{i}-\frac{g}{4} \sum_{i} s_{i}^{2}}
\end{aligned}
$$

where we have substituted (4.2) and used $\operatorname{vol}(S)=N^{N^{2} / 2} \operatorname{vol}(U(n))$ with respect to the CartanKilling metric on $\mathfrak{s}$, since $S=U(n) \otimes \mathbb{1}_{N}$. Applying the integral identity (4.58) therefore allows us to finally write the partition function as

$$
Z_{0}=\frac{1}{n !} \frac{1}{(2 \pi)^{n^{2}+n}} \mathrm{e}^{-\frac{n N^{2}}{4 g}} \int_{\mathbb{R}^{n}}[\mathrm{~d} s] \Delta(s)^{2} \mathrm{e}^{-\frac{g}{4} \sum_{i} s_{i}^{2}} .
$$

The exponential prefactor in the above expression is the Boltzmann weight of the action (3.15) evaluated on the vacuum solution. The remaining quantum fluctuation integral is the standard expression [19] for the contribution from the global minimum of the Yang-Mills action on $S^{2}$ to the $U(n)$ sphere partition function. It arises from the trivial instanton configuration with vanishing monopole charges $m_{i}=0$ in (3.17).

\subsection{Localization at maximally irreducible saddle points}

We now turn to the opposite extreme and look at the local contribution to the partition function (4.20) from a generic maximally non-degenerate critical surface. We denote this gauge orbit by

$$
\mathcal{O}_{\max }:=\mathcal{C}_{\left(n_{1}, 1\right), \ldots,\left(n_{n}, 1\right)}=\left\{g C g^{-1} \mid g \in U\left(n N-\mathrm{c}_{1}\right)\right\} \cong U\left(n N-\mathrm{c}_{1}\right) / U(1)^{n}
$$

and assume that the integers $n_{1}>n_{2}>\cdots>n_{n}$ are explicitly specified. Here we allow also $c_{1} \neq 0$ which describes sectors with non-vanishing $U(1)$ monopole number (3.13). We want to compute the integral $Z_{\max }$ in (4.20) over a local neighbourhood $\mathcal{N}_{\max }$ of $\mathcal{O}_{\max }$, which is independent of $t$ in the large $t$ limit.

We first need to find a suitable basis for the tangent space $T_{C} \mathcal{O}$ at the irreducible critical point $C$. The definition of the basis $J_{i}, \tilde{J}_{i}$ introduced in (4.46) naturally extends to include the non-trivial subspaces $E_{0}, E_{1}$ in this case with

$$
J_{i}=\mathcal{J}\left(g_{i}^{\prime}\right), \quad \tilde{J}_{j}=\mathcal{J}^{2}\left(g_{j}^{\prime}\right), \quad H_{i}=\mathcal{J}\left(h_{i}^{\prime}\right) \in \mathcal{J}(\mathfrak{h})=E_{0} \quad \text { and } \quad K_{i} \in E_{1} .
$$

for $g_{i}^{\prime}$ and $h_{i}^{\prime}$ an orthonormal basis of $\mathfrak{g} \ominus \mathfrak{h} \ominus \mathfrak{s}$ and of $\mathfrak{h} \ominus \mathfrak{s}$, respectively. The elements $K_{i}$ are assumed to form an orthonormal basis of $E_{1}$, orthogonal to $\mathcal{J}(\mathfrak{g}) \oplus \mathcal{J}^{2}(\mathfrak{g})$. Recall from Section 3.4 that $E_{0}$ and $E_{1}$ are naturally complex vector spaces, whose generators are embedded into the tangent space decomposition (3.36) as

$$
K_{i}=\left(\begin{array}{cc|cc}
0 & 0 & 0 & 0 \\
0 & 0 & X_{i} & 0 \\
\hline 0 & X_{i}^{\dagger} & 0 & 0 \\
0 & 0 & 0 & 0
\end{array}\right)
$$

and similarly for $H_{i}$. The complex structure is given by the map $\mathcal{J}$, which amounts to multiplying $X_{i}$ by i. We accordingly take the real basis $K_{i}$ to be ordered as $\left\{K_{i}\right\}=\left\{\left(\tilde{K}_{i}, \mathcal{J}\left(\tilde{K}_{i}\right)\right)\right\}$, and 
similarly for $H_{i}$. As matrices, all of the generators $H_{i}, K_{j}$ are hermitian. The corresponding dual one-forms $\beta^{i}, \gamma^{i}$ are defined as usual by

$$
\left\langle\beta^{i}, H_{j}\right\rangle=\delta^{i}{ }_{j} \quad \text { and } \quad\left\langle\gamma^{i}, K_{j}\right\rangle=\delta^{i}{ }_{j}
$$

with all other pairings equal to 0 .

We need to evaluate the pairing $\left\langle\alpha, V_{\phi}\right\rangle$. It vanishes on-shell, and identically on $\mathcal{J}^{2}(\mathfrak{g})$. Its evaluation on $\mathcal{J}(\mathfrak{g} \ominus \mathfrak{h} \ominus \mathfrak{s})$ has the form $\left\langle\alpha, \mathcal{J}\left(g_{i}^{\prime}\right)\right\rangle=f_{i}$, and as before this implies (4.49). Together with (4.30) and (4.31), it follows that the localization one-form $\alpha$ admits an expansion

$$
\alpha=f_{i} \lambda^{i}+g_{i} \beta^{i}+k_{i} \gamma^{i}
$$

where $f_{i}, g_{i}, k_{i}$ vanish on-shell. We can evaluate

$$
\mathrm{d} \alpha=\mathrm{d} f_{i} \wedge \lambda^{i}+f_{i} \mathrm{~d} \lambda^{i}+\mathrm{d} g_{i} \wedge \beta^{i}+g_{i} \mathrm{~d} \beta^{i}+\mathrm{d} k_{i} \wedge \gamma^{i}+k_{i} \mathrm{~d} \gamma^{i}
$$

using (4.44) and (4.43) to get

$$
\left\langle\mathrm{d} \alpha, H_{i} \wedge H_{j}\right\rangle=0 \quad \text { and } \quad\left\langle\mathrm{d} \alpha, K_{i} \wedge K_{j}\right\rangle=A_{i j},
$$

where

$$
A_{i j}=2 \mathrm{i} \operatorname{Tr}\left(K_{i} \operatorname{ad}_{C_{0}}\left(K_{j}\right)\right)
$$

is an antisymmetric matrix. Furthermore, $\mathrm{d} \alpha$ vanishes when evaluated on mixed terms of the form $K_{i} \wedge \mathcal{J}(g), K_{i} \wedge \mathcal{J}^{2}(g), H_{i} \wedge \mathcal{J}\left(g^{\prime}\right)$ and $H_{i} \wedge \mathcal{J}^{2}\left(g^{\prime}\right)$ with $g \in \mathfrak{g}, g^{\prime} \in \mathfrak{g} \ominus \mathfrak{h} \ominus \mathfrak{s}$. Therefore

$$
\mathrm{d} \alpha=\mathrm{d} f_{i} \wedge \lambda^{i}+\frac{1}{2} A_{i j} \gamma^{i} \wedge \gamma^{j}+O_{f}
$$

where $O_{f}$ denotes contributions which vanish on-shell such as $f_{i} \mathrm{~d} \lambda^{i}$. One then has

$$
\frac{(\mathrm{d} \alpha)^{d-d_{0}}}{\left(d-d_{0}\right) !}=\operatorname{pfaff}(A)\left(\bigwedge_{i=1}^{2 d_{1}} \gamma^{i}\right) \wedge\left(\bigwedge_{j=1}^{d-d_{0}-d_{1}} \mathrm{~d} f_{j} \wedge \lambda^{j}\right)+O_{f}
$$

where $d_{0}\left(\right.$ resp. $\left.d_{1}\right)$ is the complex dimension of the vector space $E_{0}$ (resp. $\left.E_{1}\right)$, and

$$
\operatorname{pfaff}(A)=\epsilon^{i_{1} \cdots i_{2 d_{1}}} A_{i_{1} i_{2}} \cdots A_{i_{2 d_{1}-1} i_{2 d_{1}}}
$$

is the pfaffian of the antisymmetric matrix $A=\left(A_{i j}\right)$.

Let us now recall the local geometry and define its symplectic model. The $G$-equivariant tubular neighbourhood $\mathcal{N}_{\max }$ of $\mathcal{O}_{\max }$ has an equivariant retraction [45] by a local equivariant symplectomorphism onto the local symplectic model $\mathcal{F}_{\text {max }}$, defined to be an equivariant symplectic vector bundle over $\mathcal{O}_{\text {max }}$ with fibre $\mathcal{J}^{2}(\mathfrak{g} \ominus \mathfrak{h} \ominus \mathfrak{s}) \oplus E_{1}$ which is a sub-bundle of the tangent bundle $T \mathcal{O}$ restricted to $\mathcal{O}_{\max }$. This means that the tangent space to $\mathcal{F}_{\max }$ is given by

$$
T_{C} \mathcal{O}_{\max } \oplus \mathcal{J}^{2}(\mathfrak{g} \ominus \mathfrak{h} \ominus \mathfrak{s}) \oplus E_{1} \cong E_{0} \oplus \mathcal{J}(\mathfrak{g} \ominus \mathfrak{h} \ominus \mathfrak{s}) \oplus \mathcal{J}^{2}(\mathfrak{g} \ominus \mathfrak{h} \ominus \mathfrak{s}) \oplus E_{1}=T_{C} \mathcal{O}
$$

the symplectic form on $\mathcal{F}_{\text {max }}$ is simply $\omega$, and the hamiltonian $G$-action on $\mathcal{F}_{\text {max }}$ descends from the moment map $\mu$. In physical terms, the gauge fields are split along the moduli space $\mathcal{O}_{\max }$, plus infinitesimal non-gauge variations belonging to $\mathcal{J}^{2}(\mathfrak{g} \ominus \mathfrak{h} \ominus \mathfrak{s})$ and unstable modes in the subspace $E_{1}$. Due to the presence of the localization form $\alpha$ in the action, we can restrict ourselves to this model $\mathcal{F}_{\text {max }}$ replacing $\mathcal{N}_{\text {max }}$. Identically to the case of Section 4.3 above, the canonical symplectic integral over $\mathfrak{g} \times \mathcal{N}_{\text {max }}$ will in this way reduce to an integral over $\mathfrak{s} \times \mathcal{O}_{\text {max }}$ and the localization now resembles that at an irreducible flat connection of Chern-Simons theory [27]. 
We may now proceed to calculate

$$
\begin{aligned}
& Z_{\max }= \frac{1}{\operatorname{vol}(G)} \int_{\mathfrak{g} \times \mathcal{N}_{\max }}\left[\frac{\mathrm{d} \phi}{2 \pi}\right] \exp \left(\omega+t\left(\mathrm{~d} \alpha-\mathrm{i}\left\langle\alpha, V_{\phi}\right\rangle\right)-\mathrm{i} \operatorname{Tr}\left(C_{0} \phi\right)-\frac{g^{\prime}}{2} \operatorname{Tr}\left(\phi^{2}\right)\right) \\
&= \frac{1}{\operatorname{vol}(G)} \int_{\mathfrak{g} \times \mathcal{O}_{\max } \times \mathcal{J}^{2}(\mathfrak{g} \ominus \mathfrak{h} \ominus \mathfrak{s}) \times E_{1}}\left[\frac{\mathrm{d} \phi}{2 \pi}\right] \frac{(t \mathrm{~d} \alpha)^{d-d_{0}}}{\left(d-d_{0}\right) !} \wedge \frac{\omega^{d_{0}}}{d_{0} !} \mathrm{e}^{-\mathrm{i} t\left\langle\alpha, V_{\phi}\right\rangle-\mathrm{i} \operatorname{Tr}\left(C_{0} \phi\right)-\frac{g^{\prime}}{2} \operatorname{Tr}\left(\phi^{2}\right)} \\
&= \frac{1}{\operatorname{vol}(G)} \int_{(\mathfrak{g} \ominus \mathfrak{h} \ominus \mathfrak{s}) \oplus \mathfrak{h} \oplus \mathfrak{s}}\left[\frac{\mathrm{d} \phi}{2 \pi}\right] \operatorname{pfaff}(A) \\
& \quad \times \int_{\mathcal{O}_{\max } \times \mathcal{J}^{2}(\mathfrak{g} \ominus \mathfrak{h} \ominus \mathfrak{s}) \times E_{1}} t^{d-d_{0}}\left(\bigwedge_{i=1}^{2 d_{1}} \gamma^{i}\right) \wedge\left(\bigwedge_{j=1}^{d-d_{0}-d_{1}} \mathrm{~d} f_{j} \wedge \lambda^{j}\right) \wedge \frac{\omega^{d_{0}}}{d_{0} !} \\
& \times \mathrm{e}^{-\mathrm{i} t\left(N f_{i} \phi^{i}+\left\langle\alpha, V_{\phi^{\prime}}\right\rangle\right)-\mathrm{i} \operatorname{Tr}\left(C_{0} \phi\right)-\frac{g^{\prime}}{2} \operatorname{Tr}\left(\phi^{2}\right)}
\end{aligned}
$$

with $\phi^{\prime} \in \mathfrak{h} \oplus \mathfrak{s}$. In the second line we have used the fact that $\mathrm{d} \alpha$ vanishes when evaluated on the subspace $E_{0}$, and therefore we need $d_{0}$ powers of $\omega$ to yield a non-trivial volume form. Then $(t \mathrm{~d} \alpha)^{d-d_{0}} \wedge \omega^{d_{0}}$ is the only term which survives in the large $t$ limit. We will modify this below by adding a second localization form $\alpha^{\prime}$ in order to write the localization integral in the generic form (4.20) without the symplectic two-form $\omega$.

We can now evaluate the integrals in (4.75) over $f_{i}$ in the fibre $\mathcal{J}^{2}(\mathfrak{g} \ominus \mathfrak{h} \ominus \mathfrak{s})$ and $\phi^{i} \in \mathfrak{g} \ominus \mathfrak{h} \ominus \mathfrak{s}$ as in Section 4.3 above, which localizes for $t \rightarrow \infty$ to an integral over the subspace $E_{1}$ and the gauge orbit $\mathcal{O}_{\max }$ given by

$$
\begin{aligned}
& Z_{\max }=\frac{1}{\operatorname{vol}(G)} \int_{\mathfrak{h} \oplus \mathfrak{s}}\left[\frac{\mathrm{d} \phi}{2 \pi}\right] \frac{\operatorname{pfaff}(A)}{N^{d-d_{0}-d_{1}}} \int_{\mathcal{O}_{\max } \times E_{1}} t^{d_{1}}\left(\bigwedge_{i=1}^{2 d_{1}} \gamma^{i}\right) \wedge\left(\bigwedge_{j=1}^{d-d_{0}-d_{1}} \lambda^{j}\right) \wedge \frac{\omega^{d_{0}}}{d_{0} !} \\
& \times \mathrm{e}^{-\mathrm{i} t\left\langle\alpha, V_{\phi}\right\rangle-\mathrm{i} \operatorname{Tr}\left(C_{0} \phi\right)-\frac{g^{\prime}}{2} \operatorname{Tr}\left(\phi^{2}\right)} .
\end{aligned}
$$

The gauge invariant volume form for the integration domain whose tangent space is $E_{0}$ is given by the symplectic volume form $\omega^{d_{0}} / d_{0}$ !, since $\mathrm{d} \alpha$ vanishes on $E_{0}$, but this will be modified below. It remains to compute the integral over $E_{1}$. Upon evaluating $\left\langle\alpha, V_{\phi}\right\rangle$ at second order on $E_{1}$, i.e. away from the critical surface, we will find below that this pairing becomes a quadratic form which leads to a localization through a gaussian integral. However, to evaluate it explicitly it is easier to first localize the integral over $E_{0}$, which presently is a complicated non-gaussian integral which does not admit a gaussian approximation at $t \rightarrow \infty$ and is difficult to evaluate in a closed analytic form. But this can be done by adapting a trick taken from [27], which amounts to adding a further suitable localization one-form $\alpha^{\prime}$, or equivalently a cohomologically trivial form $Q \alpha^{\prime}$, to the action

in (4.20). Indeed, we may compute $Z_{\max }$ using any other invariant form $\alpha^{\prime}$ which is homotopic to $\alpha$ on the open neighbourhood $\mathcal{N}_{\max }$. The one-form $\alpha^{\prime}$ need only be non-vanishing on $E_{0} \subset \mathcal{N}_{\max }$, as the other integrals can be directly carried out.

\section{The localization form $\alpha^{\prime}$}

In order to evaluate the integrals over $E_{0}$ and $\mathfrak{h}$, following [27] we introduce an additional localization term $\exp \left(t Q \alpha^{\prime}\right)$ in the partition function with

$$
\alpha^{\prime}:=-\left.\mathrm{i} \operatorname{Tr}(\theta \phi)\right|_{E_{0}}=-\left.\frac{2}{N} \mathcal{J} \mathrm{d} \operatorname{Tr}(C \phi)\right|_{E_{0}} .
$$

The projection onto $E_{0}$ is equivalent to projecting $\phi \in \mathfrak{g}$ onto $\mathfrak{h}$. This one-form is equivariant on-shell, and it can be extended to the $G$-equivariant tubular neighbourhood $\mathcal{N}_{\max }$ of the critical 
surface $\mathcal{O}_{\max }$ as follows. On the tangent space $\mathcal{J}(\mathfrak{g} \ominus \mathfrak{h} \ominus \mathfrak{s}) \oplus E_{0}$ of $T \mathcal{O}_{\max }$ (4.74) there is an equivariant projection onto the subspace $E_{0}$. In this way $\alpha^{\prime}$ is properly defined on the local model, and can hence be extended to $\mathcal{N}_{\max }$. One could also define $\alpha^{\prime}=-\left.\mathrm{i} \chi \operatorname{Tr}(\theta \phi)\right|_{E_{0}}$ using a smooth $G$-invariant cutoff function $\chi$ with support near the given saddle-point and $\chi=1$ in the tubular neighbourhood, which is globally well-defined over $\mathcal{N}_{\max }$ as an equivariant differential form. Note that $t_{1} \alpha+t_{2} \alpha^{\prime}$ vanishes only on the original critical points for any $t_{1}, t_{2} \in \mathbb{R}$ with $t_{1} \neq 0$, and no new ones are introduced. Then our previous computation (4.17) would essentially go through, since $\alpha^{\prime}$ vanishes on $\mathcal{J}(\mathfrak{g} \ominus \mathfrak{h} \ominus \mathfrak{s})$ and there are no critical points where $\mathrm{d} \chi \neq 0$. It is therefore just as good a localization form to use as $\alpha$ is. It follows that the modification of the canonical symplectic integral over $\mathcal{N}_{\max }$ given by

$$
Z_{\max }=\frac{1}{\operatorname{vol}(G)} \int_{\mathfrak{g} \times \mathcal{N}_{\max }}\left[\frac{\mathrm{d} \phi}{2 \pi}\right] \exp \left(\omega+t_{1} Q \alpha+t_{2} Q \alpha^{\prime}-\mathrm{i} \operatorname{Tr}\left(C_{0} \phi\right)-\frac{g^{\prime}}{2} \operatorname{Tr}\left(\phi^{2}\right)\right)
$$

is independent of both $t_{1}, t_{2} \in \mathbb{R}$. Then $\alpha^{\prime}$ will localize the integral over $\mathfrak{h} \subset \mathfrak{g}$ as well as the integral over the unstable modes in $E_{1}$, without the need to expand $\left\langle\alpha, V_{\phi}\right\rangle$ to higher order.

\section{Integration over $\mathfrak{h}$}

The new localization form $\alpha^{\prime}$ satisfies

$$
\mathrm{d} \alpha^{\prime}=\left.\mathrm{i} \operatorname{Tr}\left(\theta^{2} \phi\right)\right|_{E_{0}}=-\left.\frac{\mathrm{i}}{2} \operatorname{Tr}(\theta[\phi, \theta])\right|_{E_{0}}
$$

and

$$
\left\langle\alpha^{\prime}, V_{h_{i}}\right\rangle=-\frac{2}{N} \operatorname{Tr}\left(\mathcal{J}\left(V_{h_{i}}\right) \phi\right)=\frac{2}{N} \operatorname{Tr}\left(V_{h_{i}} \mathcal{J}(\phi)\right)=2 \operatorname{Tr}\left(H_{i} \mathcal{J}(\phi)\right)
$$

where $H_{i}=\mathcal{J}\left(h_{i}\right)$ with $h_{i}$ a basis of $\mathfrak{h}$. This produces a gaussian integral localizing $\mathfrak{h}$ to the gauge stabilizer algebra $\mathfrak{s} \cong \mathfrak{u}(1)^{n}$. To evaluate it, we will need the matrix

$$
M_{i j}:=\operatorname{Tr}\left(H_{i} H_{j}\right)
$$

which is hermitian since we take $H_{i}$ and $h_{i}$ to be hermitian. Similarly, one has

$$
\begin{aligned}
\left\langle\mathrm{d} \alpha^{\prime}, H_{i} \wedge H_{j}\right\rangle & =\frac{4 \mathrm{i}}{N^{2}} \operatorname{Tr}\left(\mathcal{J}\left(H_{i}\right)\left[s, \mathcal{J}\left(H_{j}\right)\right]\right) \\
& =-\frac{4 \mathrm{i}}{N^{2}} \operatorname{Tr}\left(H_{i}\left[s, \mathcal{J}^{2}\left(H_{j}\right)\right]\right) \\
& =\frac{4 \mathrm{i}}{N^{2}} \operatorname{Tr}\left(H_{i}\left[s, H_{j}\right]\right)=: \frac{4 \mathrm{i}}{N^{2}} \tilde{A}_{i j}
\end{aligned}
$$

where we have restricted to $\phi=s \in \mathfrak{s}$ using the localization. This implies that

$$
\mathrm{d} \alpha^{\prime}=\frac{2 \mathrm{i}}{N^{2}} \tilde{A}_{i j} \beta^{i} \wedge \beta^{j} \quad \text { and } \quad \frac{\left(\mathrm{d} \alpha^{\prime}\right)^{d_{0}}}{d_{0} !}=\left(\frac{4 \mathrm{i}}{N^{2}}\right)^{d_{0}} \operatorname{pfaff}(\tilde{A}) \bigwedge_{i=1}^{2 d_{0}} \beta^{i}
$$

To evaluate the matrices $M=\left(M_{i j}\right)$ and $\tilde{A}=\left(\tilde{A}_{i j}\right)$ above explicitly, we recall that the basis $H_{i}:=H_{k l ; i}$ (where $k, l$ are block indices) of $E_{0}$ takes the block form

$$
H_{k l ; i}=\left(\begin{array}{cc|cc}
0 & 0 & 0 & 0 \\
0 & 0 & Y_{l k ; i} & 0 \\
\hline 0 & Y_{l k ; i}^{\dagger} & 0 & 0 \\
0 & 0 & 0 & 0
\end{array}\right)=\mathcal{J}\left(h_{k l ; i}\right)
$$

where $h_{k l ; i} \in \mathfrak{h}$ is a hermitian block matrix with a similar block decomposition. They are orthogonal for different $k, l$, and we will often omit the indices $k, l$. Note that the complex structure on $E_{0}$ 
defined by the map $\mathcal{J}$ is compatible the natural complex structure on $\mathfrak{h}$. This basis is particularly useful for evaluating the pfaffian which appears in (4.83), because $\operatorname{ad}_{s}\left(H_{k l ; i}\right)$ for $s \in \mathfrak{s}$ acts as multiplication by $\left(s_{k}-s_{l}\right)$ in the upper-right blocks of (4.84). It follows that

$$
\mathrm{i} \operatorname{ad}_{C_{0}}\left(H_{k l ; i}\right)=c_{l k} \mathcal{J}\left(H_{k l ; i}\right) \quad \text { and } \quad \mathrm{i}_{\operatorname{ad}_{s}}\left(H_{k l ; i}\right)=\left(s_{l}-s_{k}\right) \mathcal{J}\left(H_{k l ; i}\right)
$$

where the eigenvalues $c_{l k}>0$ are defined in (3.39). These formulas hold only for $k>l$, and analogous statements are true for the subspace $E_{1}$.

We can choose an orthogonal basis $Y_{i}$ such that $G_{i j}=2 \operatorname{Tr}\left(Y_{i} Y_{j}^{\dagger}\right)$ is diagonal, as $G_{i j}$ is a hermitian matrix. Then

$$
\begin{aligned}
\operatorname{Tr}\left(H_{i} H_{j}\right) & =\operatorname{Tr}\left(Y_{i} Y_{j}^{\dagger}+Y_{i}^{\dagger} Y_{j}\right)=G_{i j}, \\
\operatorname{Tr}\left(H_{i} \mathcal{J}\left(H_{j}\right)\right) & =\operatorname{Tr}\left(\mathrm{i} Y_{i} Y_{j}^{\dagger}-\mathrm{i} Y_{i}^{\dagger} Y_{j}\right)=0 .
\end{aligned}
$$

This means that the symmetric matrix $M=\left(M_{i j}\right)$ in (4.81) has the block decomposition

$$
M=\left(\begin{array}{cc}
G & 0 \\
0 & G
\end{array}\right)
$$

in the basis $\left(\tilde{H}_{i}, \mathcal{J}\left(\tilde{H}_{i}\right)\right)$, and similarly the matrix $\tilde{A}$ in (4.82) is given by

$$
\begin{aligned}
\tilde{A}_{i j} & =\operatorname{Tr}\left(H_{i} \operatorname{ad}_{s}\left(H_{j}\right)\right) \\
& =-\mathrm{i}\left(s_{l}-s_{k}\right) \operatorname{Tr}\left(H_{i} \mathcal{J}\left(H_{j}\right)\right)=-\mathrm{i}\left(s_{k}-s_{l}\right)\left(\begin{array}{cc}
0 & G \\
-G & 0
\end{array}\right)_{i j} .
\end{aligned}
$$

We can read off the pfaffian from this expression and use (4.87) to write it as

$$
\operatorname{pfaff}(\tilde{A})=(-\mathrm{i})^{d_{0}} \sqrt{\operatorname{det}(M)} \prod_{k>l}\left(s_{k}-s_{l}\right)^{\left|n_{k}-n_{l}\right|+1} .
$$

We can now evaluate the localization integral

$$
\int_{\mathfrak{h}}\left[\frac{\mathrm{d} \phi}{2 \pi}\right] t_{2}^{d_{0}} \frac{\left(\mathrm{d} \alpha^{\prime}\right)^{d_{0}}}{d_{0} !} \mathrm{e}^{-\mathrm{i} t_{2}\left\langle\alpha^{\prime}, V_{\phi}\right\rangle}=\left(\frac{4 \mathrm{i}}{N^{2}}\right)^{d_{0}} \int_{\mathfrak{h}}\left[\frac{\mathrm{d} \phi}{2 \pi}\right] t_{2}^{d_{0}} \operatorname{pfaff}(\tilde{A}) \mathrm{e}^{-2 \mathrm{i} t_{2} \phi^{i} M_{i j} \phi^{j}} \bigwedge_{i=1}^{2 d_{0}} \beta^{i}
$$

where $\phi=\phi^{i} h_{i}=\phi^{k l ; i} h_{k l ; i}$. The oscillatory gaussian integral is defined by analytic continuation $t_{2} \rightarrow t_{2}-\mathrm{i} \varepsilon$ for a small positive parameter $\varepsilon$, which we are free to do as the partition function is formally independent of $t_{2}$. With this continuation understood and a suitable orientation of the vector space $\mathfrak{h}$, we readily compute

$$
\begin{aligned}
\int_{\mathfrak{h}}\left[\frac{\mathrm{d} \phi}{2 \pi}\right] t_{2}^{d_{0}} \frac{\left(\mathrm{d} \alpha^{\prime}\right)^{d_{0}}}{d_{0} !} \mathrm{e}^{-\mathrm{i} t_{2}\left\langle\alpha^{\prime}, V_{\phi}\right\rangle} & =\left(\frac{4 \mathrm{i}}{N^{2}}\right)^{d_{0}}\left(\frac{1}{2 \pi}\right)^{2 d_{0}}\left(-\frac{\pi}{2 \mathrm{i}}\right)^{d_{0}} \frac{\operatorname{pfaff}(\tilde{A})}{\sqrt{\operatorname{det}(M)}} \bigwedge_{i=1}^{2 d_{0}} \beta^{i} \\
& =\frac{\mathrm{i}^{d_{0}}}{\left(2 \pi N^{2}\right)^{d_{0}}} \prod_{k>l}\left(s_{k}-s_{l}\right)^{\left|n_{k}-n_{l}\right|+1} \bigwedge_{i=1}^{2 d_{0}} \beta^{i}
\end{aligned}
$$

This integral thus produces a measure on $\mathfrak{s}$ which we will use below to perform the remaining integral over the stabilizer. 


\section{Integration over $E_{1}$}

Now that the $\phi$-integration in (4.76) is localized onto $\mathfrak{s}$, we can proceed to evaluate the integral over $E_{1}$. This space has a basis $K_{i}$ with block decomposition $K_{k l ; i}$ similar to (4.84) for $n \geq k>l \geq 1$ (for $k<l$ the $K_{k l ; i}$ do not exist), which are non-vanishing if $n_{k}>n_{l}+1$. We need to evaluate $\left\langle\alpha, V_{s}\right\rangle$ for $s \in \mathfrak{s}$ up to second order in the fluctuations about the critical point in $E_{1}$, which is non-tangential to the gauge orbit $\mathcal{O}_{\max }$. For this, we introduce real linear coordinates $x^{i}, y^{i}, i=1, \ldots, d_{1}$ on $E_{1}$ such that a generic vector $V_{\Psi} \in E_{1}$ is parametrized as

$$
V_{\Psi}=\left(x^{i} K_{i}, y^{i} \mathcal{J}\left(K_{i}\right)\right)=\left(\begin{array}{cc|cc}
0 & 0 & 0 & 0 \\
0 & 0 & z^{i} X_{i} & 0 \\
\hline 0 & \bar{z}^{i} X_{i}^{\dagger} & 0 & 0 \\
0 & 0 & 0 & 0
\end{array}\right)
$$

where we have introduced complex coordinates $z^{i}=x^{i}+\mathrm{i} y^{i}$. Then $\gamma^{i}=\mathrm{d} x^{i}$ and $\gamma^{i+d_{1}}=\mathrm{d} y^{i}$ for $i=1, \ldots, d_{1}$.

As above, we can choose coordinates such that $G_{i j}=2 \operatorname{Tr}\left(X_{i} X_{j}^{\dagger}\right)$ is diagonal. Then (4.37) gives

$$
\left\langle\alpha, V_{s}\right\rangle=-\operatorname{Tr}\left(\operatorname{ad}_{s}\left(V_{\Psi}\right) \operatorname{ad}_{C_{0}}\left(V_{\Psi}\right)\right)=\left(x^{i}, y^{i}\right) \tilde{M}_{i j}(s)\left(\begin{array}{c}
x^{j} \\
y^{j}
\end{array}\right)
$$

to second order, where

$$
\begin{aligned}
\tilde{M}_{i j}(s) & =\operatorname{Tr}\left(K_{i} \operatorname{ad}_{s} \operatorname{ad}_{C_{0}}\left(K_{j}\right)\right) \\
& =\left(s_{k}-s_{l}\right) c_{k l} \operatorname{Tr}\left(K_{i} K_{j}\right)=\left(s_{k}-s_{l}\right) c_{k l}\left(\begin{array}{cc}
G & 0 \\
0 & G
\end{array}\right)_{i j}
\end{aligned}
$$

is a symmetric matrix and we have used the obvious analog of (4.85) for the basis $K_{i}$. Similarly, the antisymmetric matrix $A$ in (4.70) can be expressed as

$$
A_{i j}=2 \mathrm{i} \operatorname{Tr}\left(K_{k l ; i} \operatorname{ad}_{C_{0}}\left(K_{k l ; j}\right)\right)=2 c_{l k} \operatorname{Tr}\left(K_{k l ; i} \mathcal{J}\left(K_{k l ; j}\right)\right)=2 c_{k l}\left(\begin{array}{cc}
0 & G \\
-G & 0
\end{array}\right)_{i j},
$$

and using (4.94) its pfaffian is therefore given by

$$
\operatorname{pfaff}(A)=2^{d_{1}} \sqrt{\operatorname{det}(\tilde{M}(s))} \prod_{k>l}\left(s_{k}-s_{l}\right)^{1-\left|n_{k}-n_{l}\right|} .
$$

The pfaffians $\operatorname{pfaff}(\tilde{A})$ and $\operatorname{pfaff}(A)$ represent the $S$-equivariant Euler classes in $H_{S}\left(\mathcal{O}_{\max }\right)$ of equivariant bundles over $\mathcal{O}_{\max }$ with fibres $E_{0}$ and $E_{1}$, respectively, in terms of the weights $s_{k}$ for the (trivial) $S$-action on $\mathcal{O}_{\max }$. They are the typical representatives of fluctuations in equivariant localization [29,41], and they also appear in the nonabelian localization formulas of [27] and of [26]. Using the analytic continuation $t_{1} \rightarrow t_{1}-\mathrm{i} \varepsilon$ and a suitable orientation of $E_{1}$ as before, we can now evaluate the oscillatory gaussian integral

$$
\int_{E_{1}} \prod_{i=1}^{d_{1}} \mathrm{~d} x^{i} \mathrm{~d} y^{i} t_{1}^{d_{1}} \mathrm{e}^{-\mathrm{i} t_{1}\left\langle\alpha, V_{s}\right\rangle}=\left(\frac{\pi}{\mathrm{i}}\right)^{d_{1}} \frac{1}{\sqrt{\operatorname{det}(\tilde{M}(s))}} .
$$


Symplectic integral over $\mathcal{F}_{\max }$

Putting the results $(4.76),(4.91),(4.96)$ and (4.97) together, we may evaluate the large $t_{1}, t_{2}$ limit of the symplectic integral (4.78) to obtain

$$
\begin{aligned}
Z_{\max }=\frac{1}{\operatorname{vol}(G)} \int_{\mathfrak{g} \times \mathcal{F}_{\max }}\left[\frac{\mathrm{d} \phi}{2 \pi}\right] \exp \left(\mathrm{d}\left(t_{1} \alpha+t_{2} \alpha^{\prime}\right)-\mathrm{i}\left\langle t_{1} \alpha+t_{2} \alpha^{\prime}, V_{\phi}\right\rangle\right) \\
\quad \times \mathrm{e}^{-\mathrm{i} \operatorname{Tr}\left(C_{0} \phi\right)-\frac{g^{\prime}}{2} \operatorname{Tr}\left(\phi^{2}\right)} \\
=\frac{1}{\operatorname{vol}(G)}\left(\frac{\pi}{\mathrm{i}}\right)^{d_{1}} \frac{\mathrm{i} d_{0}}{\left(2 \pi N^{2}\right)^{d_{0}}} \int_{\mathfrak{s}}\left[\frac{\mathrm{d} s}{2 \pi}\right] \prod_{k>l}\left(s_{k}-s_{l}\right)^{\left|n_{k}-n_{l}\right|+1} \frac{\operatorname{pfaff}(A)}{\sqrt{\operatorname{det}(\tilde{M}(s))}} \\
\quad \times \frac{1}{N^{d-d_{0}-d_{1}}} \int_{\mathcal{O}_{\max }}\left(\bigwedge_{j=1}^{d-d_{0}-d_{1}} \lambda^{j}\right) \wedge\left(\bigwedge_{i=1}^{2 d_{0}} \beta^{i}\right) \mathrm{e}^{-\mathrm{i} \operatorname{Tr}\left(C_{0} s\right)-\frac{g^{\prime}}{2} \operatorname{Tr}\left(s^{2}\right)} \\
=\frac{1}{\operatorname{vol}(G)} \frac{\mathrm{i} d_{0}-d_{1}}{(2 \pi)^{d_{0}-d_{1}}} \prod_{k=1}^{n} \int_{\mathbb{R}^{n}}\left[\frac{\mathrm{d} s}{2 \pi}\right] \Delta(s)^{2} \mathrm{e}^{-\mathrm{i} \operatorname{Tr}\left(C_{0} s\right)-\frac{g^{\prime}}{2} \operatorname{Tr}\left(s^{2}\right)} \\
\quad \times \frac{1}{N^{d+d_{0}-d_{1}}} \int_{\mathcal{O}_{\max }}\left(\bigwedge_{j=1}^{d-d_{0}-d_{1}} \lambda^{j}\right) \wedge\left(\bigwedge_{i=1}^{2 d_{0}} \beta^{i}\right)
\end{aligned}
$$

where we have transformed the integration over $\phi=s=\operatorname{diag}\left(s_{1} \mathbb{1}_{n_{1}}, \ldots, s_{n} \mathbb{1}_{n_{n}}\right) \in \mathfrak{s}$ to an integral over $s=\left(s_{1}, \ldots, s_{n}\right) \in \mathbb{R}^{n}$. We can carry out the integral over the moduli space $\mathcal{O}_{\max }$ by observing again

$$
\frac{1}{N^{d+d_{0}-d_{1}}} \int_{\mathcal{O}_{\max }}\left(\bigwedge_{j=1}^{d-d_{0}-d_{1}} \lambda^{j}\right) \wedge\left(\bigwedge_{i=1}^{2 d_{0}} \beta^{i}\right)=\int_{G / S} \bigwedge_{j=1}^{d+d_{0}-d_{1}} \eta^{j}=\frac{\operatorname{vol}(G)}{\operatorname{vol}(S)}
$$

where $\mathcal{J}^{*}\left(\lambda^{i}\right)=\eta^{i}$ are left-invariant one-forms on the gauge group $G$. Note that (4.99) includes the integral over $E_{0}$, and $\operatorname{dim}_{\mathbb{R}}(\mathfrak{g} \ominus \mathfrak{s})=d+d_{0}-d_{1}$. We also have $\operatorname{vol}(S)=\prod_{k} 2 \pi \sqrt{n_{k}}$ in our metric on $\mathfrak{s}$, since $S=\prod_{k} U(1) \otimes \mathbb{1}_{n_{k}}$, and $C_{0}\left(n_{i}\right)=\frac{N}{2 n_{i}} \mathbb{1}_{n_{i}}$. Using furthermore $d_{0}-d_{1}=n^{2}-n$ which is an even integer, we may then bring (4.98) into the form

$$
\begin{aligned}
Z_{\max } & =\frac{\mathrm{i}^{n^{2}-n}}{(2 \pi)^{n^{2}+n}} \int_{\mathbb{R}^{n}}[\mathrm{~d} s] \Delta(s)^{2} \mathrm{e}^{-\mathrm{i} \operatorname{Tr}\left(C_{0} s\right)-\frac{g^{\prime}}{2} \operatorname{Tr}\left(s^{2}\right)} \\
& =\frac{\mathrm{i}^{n^{2}-n}}{(2 \pi)^{n^{2}+n}} \int_{\mathbb{R}^{n}}[\mathrm{~d} s] \Delta(s)^{2} \mathrm{e}^{-\frac{\mathrm{i}}{2} N \sum_{i} s_{i}-\frac{g}{4} \sum_{i} \frac{n_{i}}{N} s_{i}^{2}} \\
& =\frac{\mathrm{i}^{n^{2}-n}}{(2 \pi)^{n^{2}+n}} \frac{N^{n / 2}}{\prod_{k=1}^{n} \sqrt{n_{k}}} \int_{\mathbb{R}^{n}}[\mathrm{~d} \tilde{s}] \prod_{k>l}\left(\sqrt{\frac{N}{n_{k}}} \tilde{s}_{k}-\sqrt{\frac{N}{n_{l}}} \tilde{s}_{l}\right)^{2} \mathrm{e}^{-\frac{\mathrm{i}}{2} \sum_{i} \sqrt{\frac{N^{3}}{n_{i}}} \tilde{s}_{i}-\frac{g}{4} \sum_{i} \tilde{s}_{i}^{2}}
\end{aligned}
$$

where $\tilde{s}_{i}:=\sqrt{n_{i} / N} s_{i}$. Completing the square of the gaussian function of $\tilde{s}_{i}$ in (4.100) identifies the Boltzmann weight of the action (3.15) on the non-degenerate solution in (3.16). In the large $N$ limit, we substitute (3.17) with $\tilde{s}_{i} \approx\left(1+\frac{m_{i}}{2 N}\right) s_{i}$. Neglecting terms of order $\frac{1}{N}$ then reduces (4.100) to

$$
Z_{\max } \approx \pm \frac{1}{(2 \pi)^{n^{2}+n}} \int_{\mathbb{R}^{n}}[\mathrm{~d} s] \Delta(s)^{2} \mathrm{e}^{-\frac{\mathrm{i}}{2} N \sum_{i} s_{i}} \mathrm{e}^{\frac{\mathrm{i}}{4} \sum_{i} m_{i} s_{i}-\frac{g}{4} \sum_{i} s_{i}^{2}}
$$

and an application of the integral identity (4.58) leads to our final result

$$
Z_{\max } \approx \pm \frac{1}{(2 \pi)^{n^{2}+n}} \mathrm{e}^{-\frac{n N^{2}-m N}{4 g}} \int_{\mathbb{R}^{n}}[\mathrm{~d} s] \Delta(s)^{2} \mathrm{e}^{\frac{\mathrm{i}}{4} \sum_{i} m_{i} s_{i}-\frac{g}{4} \sum_{i} s_{i}^{2}}
$$


The exponential prefactor in this formula exhibits the shift of the vacuum action, corresponding to the modification of the trace constraint (2.22) to (3.13), by the Chern class $c_{1}=m=\sum_{i} m_{i}$. The remaining contributions coincide with the classical result [19] for the contribution to the $U(n)$ sphere partition function from the Yang-Mills instanton on $S^{2}$ specified by the configuration of magnetic monopole charges $m_{1}, \ldots, m_{n} \in \mathbb{Z}$. In particular, using the standard manipulation of [19] one can change integration variables in (4.102) to identify the anticipated Boltzmann weight of the action (3.20).

\section{Abelianization}

In the following sections we will describe an alternative technique of evaluating the partition function of $U(n)$ Yang-Mills theory on the fuzzy sphere $S_{N}^{2}$, within the framework of our symplectic model. This method can be regarded as a finite-dimensional version of the technique of abelianization for ordinary Yang-Mills theory in two dimensions [28], which can be used to derive the strong-coupling expansion of the gauge theory and agrees with the nonabelian localization. The advantage of this formalism is that it captures all classical contributions to the partition function in a single go and for any $N$, in contrast to nonabelian localization which requires analysis of each type of critical point individually and only yields tractable expressions in the large $N$ classical limit. Its downfall is that it leads to somewhat cumbersome expressions for the partition function which arise from a rather different sort of localization. This is analogous to the case of gauge theory on the two-dimensional noncommutative torus whose strong-coupling expansion involves the addition of infinitely many higher Casimir operators to the usual Migdal formula [34], or its matrix model regularization which is given by a complicated combinatorial formula [33]. This complexity makes it difficult to explicitly extract the contributions from fuzzy sphere instantons, and we will examine this problem more thoroughly in the next section. Here we shall derive in detail our alternative abelianized formula for the partition function (4.1), representing yet another new solution for quantum gauge theory on the fuzzy sphere.

Let us start from the partition function in the form (4.3). The crucial observation is that the function $f: \mathfrak{g} \rightarrow \mathbb{R}$ defined by the symplectic integral

$$
f(\phi):=\frac{1}{\operatorname{vol}(G)} \int_{\mathcal{O}(\Xi)} \exp \left(\omega-\mathrm{i} \operatorname{Tr}\left(C_{0} \phi\right)-\frac{g^{\prime}}{2} \operatorname{Tr}\left(\phi^{2}\right)\right)
$$

is gauge invariant. Analogously to what we did in Section 4.3, we may therefore apply the Weyl integration formula (4.56) which reduces its integral over the gauge algebra $\mathfrak{g}=\mathfrak{u}(n N)$ to an integral over the Lie algebra $\mathfrak{u}(1)^{n N}$ of the maximal torus $T=U(1)^{n N}$ of $G=U(n N)$. This rewriting of the $\phi$-integral in (4.3) is called diagonalization or abelianization, and it can be thought of as the eigenvalue representation of the gauge theory regarded as a matrix model. In this way we may bring the partition function into the form

$$
Z=\frac{1}{(n N) !} \int_{\mathbb{R}^{n N}}\left[\frac{\mathrm{d} p}{2 \pi}\right] \mathrm{e}^{-\frac{g^{\prime}}{4} \operatorname{Tr}\left(p^{2}\right)} \Delta(p)^{2} Z_{\mathcal{O}}(p)
$$

where

$$
Z_{\mathcal{O}}(p)=\int_{\mathcal{O}(\Xi)} \exp \left(\omega-\frac{\mathrm{i}}{2} \operatorname{Tr}(p C)\right)
$$

is the Fourier transform of the orbit $\mathcal{O}(\Xi)$ and we have identified $(n N)$-vectors with diagonal matrices $p=\operatorname{diag}\left(p_{1}, \ldots, p_{n N}\right) \otimes \sigma^{0}$.

Localization can then be applied to the symplectic integral (5.3) in three different ways, by: 
1. Considering $p \in \mathfrak{u}(\mathcal{N})$ and observe that $Z_{\mathcal{O}}(p)$ can be considered as being invariant under $p \rightarrow U^{-1} p U$ for $U \in U(\mathcal{N})$. One can then evaluate the integral over the orbit space $\mathcal{O}(\Xi)$ directly using the Itzykson-Zuber formula (6.1) for the unitary group $U(\mathcal{N})$. This is essentially the calculation that was carried out in [9], which is adapted to the present formulation in Section 6. It amounts to an abelian localization of the original orbit integral via the Duistermaat-Heckman theorem.

2. Considering $p \in \mathfrak{u}(n N) \otimes \sigma^{0}$ and apply abelian localization to the maximal torus $T$ of the gauge group $G=U(n N)$. This will be elaborated in detail in Section 7, taking advantage of a suitable polar decomposition of the orbit space. This in turn will involve a localization onto the radial $U\left(N_{+}\right) \times U\left(N_{-}\right)$-foliation, accompanied by a fluctuation integral over the moduli space of symplectic leaves.

3. Adding a localization form $Q \alpha$ as in Section 4, and applying nonabelian localization techniques to write the partition function as a sum over local contributions from Yang-Mills critical points.

Technique 3 here was of course dealt with at length in Section 4, and will be compared in some detail to the other two approaches below. Comparison with technique 1 first is interesting in its own right

as a comparison between the matrix model approach of [9] to gauge theory on $S_{N}^{2}$ and the results of the present paper. It is also a useful warm-up to the abelianization approach of technique 2 which shares some of its qualitative features. We will find that the abelianization technique through the polar decomposition of the configuration space exploits the radial coordinates in a rather explicit way to describe the local geometry of Yang-Mills critical surfaces, and it may also find useful applications in related considerations.

\section{$6 \quad$ Itzykson-Zuber localization on the configuration space}

The integral (5.3) can be evaluated immediately using the Itzykson-Zuber formula [46], which we briefly recall. If $X, Y$ are $m \times m$ hermitian matrices with nondegenerate eigenvalues $x_{i}, y_{i} \in \mathbb{R}$, $i=1, \ldots, m$, then one has

$$
\int_{U(m)}[\mathrm{d} U] \exp \left(\frac{\mathrm{i} N}{s} \operatorname{Tr}\left(X U Y U^{\dagger}\right)\right)=c_{N}(m, s) \frac{\operatorname{det}_{1 \leq i, j \leq m}\left(\mathrm{e}^{\frac{\mathrm{i} N}{s} x_{i} y_{j}}\right)}{\Delta(x) \Delta(y)}
$$

where for $m \in \mathbb{N}$ and $s \in \mathbb{C}$ we have defined

$$
c_{N}(m, s):=\operatorname{vol}(U(m))(\mathrm{i} N / s)^{-m(m-1) / 2} \prod_{k=1}^{m-1} k !
$$

Applied to the present situation for $U(\mathcal{N})$, this yields

$$
\begin{aligned}
& Z_{\mathcal{O}}(p)=\frac{1}{\operatorname{vol}\left(U\left(N_{+}\right)\right) \operatorname{vol}\left(U\left(N_{-}\right)\right)} \int_{U(\mathcal{N})}[\mathrm{d} U] \exp \left(-\frac{\mathrm{i}}{2} \operatorname{Tr}\left(U^{-1} \Xi U \Phi\right)\right) \\
& =c_{1}^{\prime}(\mathcal{N}, 2) \frac{\operatorname{det}_{1 \leq i, j \leq \mathcal{N}}\left(\mathrm{e}^{-\frac{i}{2} \Xi_{i} \Phi_{j}}\right)}{\Delta(\Xi) \Delta(\Phi)}
\end{aligned}
$$

where $\Phi=\operatorname{diag}\left(p_{1}, \ldots, p_{n N}\right) \otimes \sigma^{0}$ and $c_{1}^{\prime}(\mathcal{N}, 2):=c_{1}(\mathcal{N}, 2) / \operatorname{vol}\left(U\left(N_{+}\right)\right) \operatorname{vol}\left(U\left(N_{-}\right)\right)$. This formula can be understood as an abelian localization with respect to the action of the maximal torus group 
$U(1)^{\mathcal{N}}$ on the flag manifold $U(\mathcal{N}) / U(1)^{\mathcal{N}}[29]$. The corresponding fixed points are the solutions of the equation

$$
[C, \Phi]=0,
$$

which are the saddle-points of the Itzykson-Zuber integral, and the expansion of the determinant in (6.3) into a sum over permutations $\pi \in \mathfrak{S}_{\mathcal{N}}$ gives the sum over critical points in the localization formula. This is completely analogous to the abelianized localization of Section 7. However, the expression (6.3) is formal as it stands because both sets of eigenvalues $\Xi_{i}$ and $\Phi_{i}$ are degenerate, and correspondingly the critical surfaces are in fact nontrivial spaces. Therefore (6.3) has to be defined using an appropriate limiting procedure which removes the degeneracy.

The partition function (4.3) is then given by

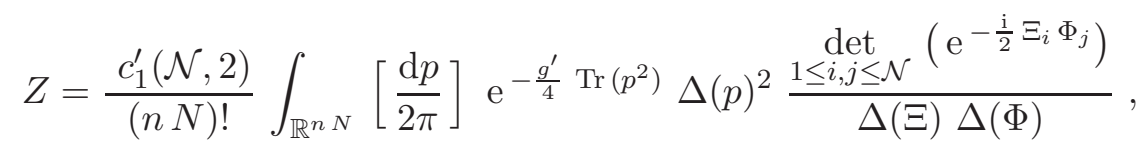

where the set of eigenvalues $\Phi_{i}$ of $\Phi$ consists of two copies of $\left(p_{1}, \ldots, p_{n N}\right)$ and is therefore highly degenerate. While this explicit formula in terms of an $n N$-dimensional integral is very appealing, the ratio of degenerate determinants in (6.5) makes it difficult to evaluate explicitly [9], and its combinatorial expansion is even more intricate than that of Section 7.3. Thus far only an asymptotic analysis (of a slightly modified integral) has been made possible in [9]. The reason for this complexity is the fact that, without the addition of a suitable localization form $Q \alpha$ to the path integral (4.3), the localization is onto the solutions of the equation (6.4) in $\mathcal{O}$ which are not related

to the critical surfaces of the Yang-Mills action in any simple way. This will be explored in more detail below.

\section{$7 \quad$ Abelian localization and radial coordinates}

We now return to the symplectic orbit integral (5.3), and observe that it fulfills the conditions of the Duistermaat-Heckman theorem, or equivalently the abelian version of the localization theorem of Section 4.1. Therefore, we have mapped the original nonabelian localization problem to the simpler problem of abelian localization. Indeed, $\left\langle\mu_{T}(C), p\right\rangle=\operatorname{Tr}(p C)$ is just the restriction of the moment map $\mu: \mathcal{O}(\Xi) \rightarrow \mathfrak{u}(\mathcal{N})^{\vee}$ to the maximal torus $T$ of the gauge group $G$. The torus action on the orbit space $\mathcal{O}(\Xi)$ is the restriction of the adjoint $G$-action given by

$$
C \longmapsto P C P^{-1}
$$

for $C=C_{\mu} \otimes \sigma^{\mu}=U \Xi U^{-1} \in \mathcal{O}(\Xi), U \in U(\mathcal{N})$ and $P \in T$. To compute the corresponding localization formula we need the fixed points of this $T$-action. They are given by those $C \in \mathcal{O}(\Xi)$ which commute with the $T$-action generated by the element $p \in \mathfrak{u}(1)^{n N}$, so that

$$
[C, p]=0 .
$$

This equation will be studied in detail in Section 8. It is solved by those $U \in U(\mathcal{N})$ for which $U^{-1} P U$ lies in the stabilizer subgroup $U\left(n N_{+}\right) \times U\left(n N_{-}\right) \subset U(\mathcal{N})$ of the element $\Xi$ (with $N_{ \pm}:=N \pm 1$ as before). The saddle points $U$ are generically also labelled by permutation matrices $\Sigma \in U(\mathcal{N})$ representing elements $\pi \in \mathfrak{S}_{n N}$. On the configuration space $\mathcal{O}$, the saddle point equation (7.2) means that $C$ commutes with the characteristic projectors of $p$, i.e. $C$ has the same block decomposition as $p$.

The Fourier transform (5.3) will thus generically localize onto a subspace of $U\left(n N_{+}\right) \times U\left(n N_{-}\right)$ in $\mathcal{O}$. It may be evaluated with the help of the degenerate version of the Duistermaat-Heckman 
theorem [29], which expresses it in terms of an integral over the critical submanifold $U\left(n N_{+}\right) \times$ $U\left(n N_{-}\right)$with the quantum fluctuation determinants determined by the $T$-equivariant Euler class of the normal bundle to the stabilizer [41]. While this can be worked out in principle, it is rather cumbersome to do in practise. Instead we will proceed in a more direct fashion by exploiting some further geometrical properties of the configuration space $\mathcal{O}$, which in the next section will be related to the local symplectic geometry near each Yang-Mills critical point as analysed at length in Section 3. This explicit calculation will justify the abelianized localization a priori, with the quantum fluctuation determinants given by integrals over symplectic leaves of a foliation of the configuration space parametrized by abelian subspaces of the tangent spaces to $\mathcal{O}$. The symplectic integral (5.3) could also be analysed using Fourier transform techniques along with the Guillemin-Lerman-Sternberg theorem [47], as in [23-25], but this leads to much more complicated combinatorial expressions than the ones we derive.

\subsection{Polar decomposition of the configuration space}

The key step in the evaluation of (5.3) is the introduction of radial coordinates on the orbit space (see [36-38] for details). Let us go back to the Cartan decomposition (3.21) at a given point $C \in \mathcal{O}$. Let $\mathfrak{t}$ be a maximal abelian subalgebra in the tangent space $T_{C} \mathcal{O} \cong \operatorname{ker}\left(\mathcal{J}^{2}+\mathbb{1}_{\mathcal{N}}\right)$. Then the radial coordinates on the orbit space $\mathcal{O}$ are given by

$$
U=V R V^{-1}=V R \mathrm{j}\left(V^{-1}\right)
$$

where $V \in U\left(n N_{+}\right) \times U\left(n N_{-}\right)$, modulo elements of the centralizer of $\mathfrak{t}$, and $R \in \exp (\mathfrak{t})$ up to the adjoint action of the Weyl group of the restricted root system of the irreducible symmetric space $\mathcal{O}$. By definition, they satisfy the respective commutation and anticommutation relations

$$
V \Xi=\Xi V \quad \text { and } \quad R \Xi=\Xi R^{-1} \text {. }
$$

The corresponding covariant coordinate $C \in \mathcal{O}(\Xi)$ is then given by

$$
\begin{aligned}
C & =U \Xi U^{-1} \\
& =V R \Xi R^{-1} V^{-1} \\
& =\frac{1}{2} V\left(R^{2} \Xi+\Xi R^{-2}\right) V^{-1}=\frac{1}{2}\left(V R^{2} V^{-1} \Xi+\Xi V R^{-2} V^{-1}\right) .
\end{aligned}
$$

The jacobian for the change of invariant integration measure on $\mathcal{O}$ can be computed by standard techniques with the result

$$
\mathrm{d} C=r(n, N)[\mathrm{d} V] \prod_{i=1}^{\operatorname{dim}(\mathfrak{t})} \mathrm{d} r_{i} \prod_{\alpha>0}|\sin (\alpha, \log R)|^{m_{\alpha}}
$$

where $^{4}$

$$
r(n, N)=\frac{\operatorname{vol}(U(\mathcal{N}))}{\operatorname{vol}\left(U\left(n N_{+}\right)\right)^{2} \operatorname{vol}\left(U\left(n N_{-}\right)\right)^{2}} \frac{2^{n^{2}\left(N^{2}-1\right) / 2}}{2^{n(N-2 n N-3) / 2}} .
$$

The radial coordinates $r_{i} \in\left[0, \frac{\pi}{2}\right]$ are the eigenvalues of $U$, while $V$ are the angular coordinates with $[\mathrm{d} V]$ denoting the standard invariant Haar measure. The second product runs over positive roots of the restricted root lattice on $\mathcal{O}$, and $m_{\alpha}$ is the multiplicity of the root $\alpha$ in the Cartan decomposition (3.21). The pairing is defined by choosing an orthonormal basis $\vec{e}_{i}$ in weight space

\footnotetext{
${ }^{4}$ The normalization constant $r(n, N)$ is determined by the requirement $\int_{\mathcal{O}} \mathrm{d} C=\operatorname{vol}(\mathcal{O})$.
} 
and identifying a root vector $\alpha$ with the dual element $\alpha^{\vee}=\sum_{i} \alpha_{i} \vec{e}_{i}$. Then $(\alpha, \log R)=\sum_{i} \alpha_{i} r_{i}$. This polar decomposition defines a foliation of the configuration space $\mathcal{O}$ by conjugacy classes under the adjoint action of the stabilizer subgroup. The radial symplectic leaves $\mathcal{L}(R)$ of this foliation are parametrized by the abelian Lie group $\exp (\mathfrak{t})$.

Let us make this decomposition more explicit using the known data for the symmetric space (2.23) [37]. The restricted root lattice is given by the root system $B C_{n N_{-}}=B_{n N_{-}} \cup C_{n N_{-}}$which has positive weights $\vec{e}_{i} \pm \vec{e}_{j}, 2 \vec{e}_{i}$ and $\vec{e}_{i}$ with $i, j=1, \ldots, n N_{-}, i<j$. The corresponding multiplicities are $m_{\vec{e}_{i} \pm \vec{e}_{j}}=2, m_{2 \vec{e}_{i}}=1$ and $m_{\vec{e}_{i}}=4 n$. The gauge invariant volume form on $\mathcal{O}$ thereby becomes

$$
\mathrm{d} C=r(n, N)[\mathrm{d} V] \prod_{i=1}^{n N_{-}} \mathrm{d} r_{i} \sin 2 r_{i} \sin ^{4 n} r_{i} \prod_{i<j} \sin ^{2}\left(r_{i}-r_{j}\right) \sin ^{2}\left(r_{i}+r_{j}\right) .
$$

Using the trigonometric identities

$$
\sin \left(r_{i}-r_{j}\right) \sin \left(r_{i}+r_{j}\right)=\frac{1}{2}\left(\cos 2 r_{j}-\cos 2 r_{i}\right) \quad \text { and } \quad \sin ^{2} r_{i}=\frac{1}{2}\left(1-\cos 2 r_{i}\right),
$$

and defining $\lambda_{i}:=\cos 2 r_{i} \in[-1,1]$, we may bring the measure to the form

$$
\mathrm{d} C=\frac{r(n, N)}{2^{n^{2}\left(N^{2}-1\right)}}[\mathrm{d} V] \Delta(\lambda)^{2} \prod_{i=1}^{n N_{-}} \mathrm{d} \lambda_{i}\left(1-\lambda_{i}\right)^{2 n} .
$$

A convenient choice for the radial coordinates is provided by setting

$$
\rho:=\operatorname{diag}\left(r_{1}, \ldots, r_{n N_{-}}\right)
$$

and defining

$$
R=\operatorname{diag}\left(\sigma^{0} \otimes \mathbb{1}_{n}, \exp \left(\mathrm{i} \sigma^{1} \otimes \rho\right)\right)=\operatorname{diag}\left(\sigma^{0} \otimes \mathbb{1}_{n}, \sigma^{0} \otimes \cos (\rho)+\mathrm{i} \sigma^{1} \otimes \sin (\rho)\right) .
$$

We also choose a basis in which

$$
\Xi=\frac{N}{2} \operatorname{diag}\left(\mathbb{1}_{n N_{+}},-\mathbb{1}_{n N_{-}}\right)=\frac{N}{2} \operatorname{diag}\left(\sigma^{0} \otimes \mathbb{1}_{n}, \sigma^{3} \otimes \mathbb{1}_{n N_{-}}\right)
$$

and $V \in U\left(n N_{+}\right) \times U\left(n N_{-}\right)$is given by

$$
V=\operatorname{diag}\left(V_{+}, V_{-}\right),
$$

with $V_{ \pm} \in U\left(n N_{ \pm}\right)$and $[\mathrm{d} V]=\left[\mathrm{d} V_{+}\right]\left[\mathrm{d} V_{-}\right]$. The relations (7.4) are then automatically satisfied.

\subsection{Evaluation of the abelianized partition function: $U(1)$ gauge theory}

We will now explicitly evaluate the Fourier transform (5.3), beginning with the abelian case $n=1$. Using (7.5) and (7.11)-(7.14), it is straightforward to work out the abelian moment map in (5.3) with the result

$$
\begin{aligned}
\left\langle\mu_{T}(C), p\right\rangle= & \operatorname{Tr}\left(p U \Xi U^{-1}\right) \\
= & \frac{1}{2} \operatorname{Tr}\left(p \Xi V\left(R^{2}+R^{-2}\right) V^{-1}\right) \\
= & \operatorname{Tr}\left(p \Xi V \operatorname{diag}\left(\sigma^{0}, \cos \left(2 \sigma^{1} \otimes \rho\right)\right) V^{-1}\right) \\
= & \frac{N}{2} \operatorname{Tr}\left(\operatorname{diag}\left(p_{1} \sigma^{0}, p_{2}, \ldots, p_{N}\right) V_{+} \operatorname{diag}\left(\sigma^{0}, \lambda_{1}, \ldots, \lambda_{N_{-}}\right) V_{+}^{-1}\right) \\
& -\frac{N}{2} \operatorname{Tr}\left(\operatorname{diag}\left(p_{2}, \ldots, p_{N}\right) V_{-} \operatorname{diag}\left(\lambda_{1}, \ldots, \lambda_{N_{-}}\right) V_{-}^{-1}\right)
\end{aligned}
$$


where we have used an inconsequential redefinition of the unitary matrix $V_{+}$by multiplication with an appropriate permutation matrix. Upon substitution into (5.3), we see that the two angular integrals decouple from each other.

The integral over $V_{-} \in U\left(N_{-}\right)$is now easily evaluated with the help of (6.1) with the result

$$
\frac{c_{N}\left(N_{-}, 4\right)}{\Delta\left(p_{2}, \ldots, p_{N}\right) \Delta(\lambda)} \sum_{\pi_{-} \in \mathfrak{S}_{N_{-}}} \operatorname{sgn}\left(\pi_{-}\right) \prod_{i=1}^{N_{-}} \mathrm{e}^{\frac{\mathrm{i} N}{4} p_{i+1} \lambda_{\pi_{-}}(i)}
$$

The integral over $V_{+} \in U\left(N_{+}\right)$is more delicate since the Itzykson-Zuber formula will involve a ratio of degenerate determinants. Since both numerator and denominator of (6.1) are completely antisymmetric functions of the eigenvalues $x_{i}$ and $y_{i}$ independently, the limit where some eigenvalues coalesce gives a well-defined analytic function in $\left(x_{i}, y_{i}\right)$ because all poles are cancelled by zeroes in the determinant. We will regularize the $V_{+}$-integral by replacing the first $p_{1}$ entry in the last line of (7.15) with an auxilliary momentum variable $p_{0} \in \mathbb{R}$, the second entry of 1 with an auxilliary radial variable $\lambda_{0} \in[-1,1]$, and then afterwards take the limits $p_{0} \rightarrow p_{1}, \lambda_{0} \rightarrow 1$. Defining $\lambda_{N}:=1$, the Itzykson-Zuber formula (6.1) applied to the regularized $V_{+}$-integral yields

$$
\frac{c_{N}\left(N_{+},-4\right)}{\Delta\left(p_{0}, p_{1}, \ldots, p_{N}\right) \Delta\left(\lambda_{0}, \lambda_{1}, \ldots, \lambda_{N}\right)} \sum_{\pi_{+} \in \mathfrak{S}_{N_{+}}} \operatorname{sgn}\left(\pi_{+}\right) \mathrm{e}^{-\frac{\mathrm{i} N}{4} p_{0} \lambda_{\pi_{+}}(N)} \prod_{i=0}^{N_{-}} \mathrm{e}^{-\frac{\mathrm{i} N}{4} p_{i+1} \lambda_{\pi_{+}(i)}}
$$

Taking the limit $p_{0} \rightarrow p_{1}$ first using l'Hôpital's rule gives

$$
\begin{gathered}
\frac{\frac{\mathrm{i} N}{4} c_{N}\left(N_{+},-4\right)}{p_{1} \prod_{i=2}^{N}\left(p_{1}-p_{i}\right) \Delta(p) \Delta\left(\lambda_{0}, \lambda_{1}, \ldots, \lambda_{N}\right)} \\
\times \sum_{\pi_{+} \in \mathfrak{S}_{N_{+}}} \operatorname{sgn}\left(\pi_{+}\right) \lambda_{\pi_{+}(N)} \mathrm{e}^{-\frac{\mathrm{i} N}{4} p_{1} \lambda_{\pi_{+}(N)}} \prod_{i=0}^{N_{-}} \mathrm{e}^{-\frac{\mathrm{i} N}{4} p_{i+1} \lambda_{\pi_{+}(i)}} .
\end{gathered}
$$

Finally, taking the limit $\lambda_{0} \rightarrow 1$ again using l'Hôpital's rule yields

$$
\begin{aligned}
& \frac{-\frac{\mathrm{i} N}{4} c_{N}\left(N_{+},-4\right)}{p_{1} \prod_{i=2}^{N}\left(p_{1}-p_{i}\right) \Delta(p) \prod_{i=1}^{N_{-}}\left(1-\lambda_{i}\right)^{2} \Delta(\lambda)}
\end{aligned}
$$

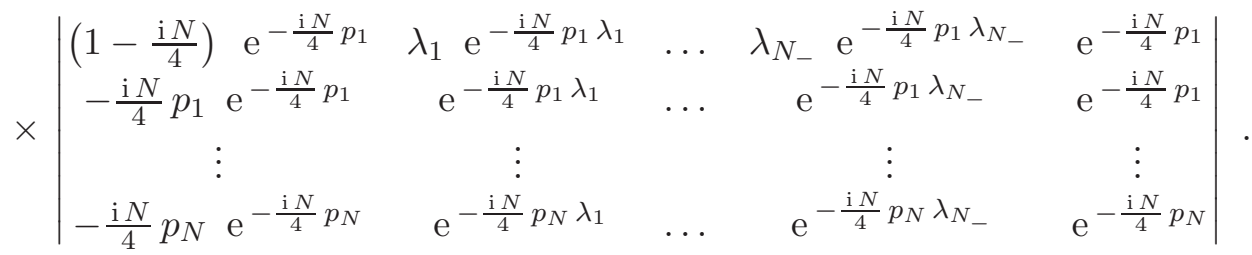

Substituting the above into (5.3) gives us the expression

$$
\begin{aligned}
& Z_{\mathcal{O}}(p)=-\frac{4 \operatorname{vol}(\mathcal{O})}{p_{1} \Delta(p)^{2}} \frac{N !(N-1) ! \prod_{k=1}^{N-2}(k !)^{2}}{(\sqrt{8} N)^{N^{2}-N}} \prod_{l=1}^{N_{-}} \int_{-1}^{1} \mathrm{~d} \lambda_{l} \sum_{\pi_{-} \in \mathfrak{S}_{N_{-}}} \operatorname{sgn}\left(\pi_{-}\right) \prod_{i=1}^{N_{-}} \mathrm{e}^{\frac{\mathrm{i} N}{4} p_{i+1} \lambda_{\pi_{-}(i)}} \\
& \times\left|\begin{array}{cccccc}
\left(1-\frac{\mathrm{i} N}{4}\right) & \mathrm{e}^{-\frac{\mathrm{i} N}{4} p_{1}} & \lambda_{1} \mathrm{e}^{-\frac{\mathrm{i} N}{4} p_{1} \lambda_{1}} & \ldots & \lambda_{N_{-}} \mathrm{e}^{-\frac{\mathrm{i} N}{4} p_{1} \lambda_{N}} & \mathrm{e}^{-\frac{\mathrm{i} N}{4} p_{1}} \\
-\frac{\mathrm{i} N}{4} p_{1} & \mathrm{e}^{-\frac{\mathrm{i} N}{4} p_{1}} & \mathrm{e}^{-\frac{\mathrm{i} N}{4} p_{1} \lambda_{1}} & \ldots & \mathrm{e}^{-\frac{\mathrm{i} N}{4} p_{1} \lambda_{N}} & \mathrm{e}^{-\frac{\mathrm{i} N}{4} p_{1}} \\
\vdots & \vdots & & \vdots & \vdots \\
-\frac{\mathrm{i} N}{4} p_{N} & \mathrm{e}^{-\frac{\mathrm{i} N}{4} p_{N}} & \mathrm{e}^{-\frac{\mathrm{i} N}{4} p_{N} \lambda_{1}} & \ldots & \mathrm{e}^{-\mathrm{i} N} p_{N} \lambda_{N_{-}} & \mathrm{e}^{-\frac{\mathrm{i} N}{4} p_{N}}
\end{array}\right| .
\end{aligned}
$$


We will now write the product of determinants in (7.20) as a single sum over the Weyl group $\mathfrak{S}_{N}$ of the original gauge symmetry group $U(N)$. For this, we embed $\mathfrak{S}_{N_{-}}$in the Weyl group $\mathfrak{S}_{N}$ as the subgroup of permutations $\pi_{-}$of $\left\{1, \ldots, N_{-}, N\right\}$ with $\pi_{-}(N)=N$. We perform a Laplace expansion of the second determinant in (7.20) into minors along the first row to write

$$
\begin{aligned}
& \left|\begin{array}{cccccc}
\left(1-\frac{\mathrm{i} N}{4}\right) & \mathrm{e}^{-\frac{\mathrm{i} N}{4} p_{1}} & \lambda_{1} \mathrm{e}^{-\frac{\mathrm{i} N}{4} p_{1} \lambda_{1}} & \ldots & \lambda_{N_{-}} \mathrm{e}^{-\frac{\mathrm{i} N}{4} p_{1} \lambda_{N_{-}}} & \mathrm{e}^{-\frac{\mathrm{i} N}{4} p_{1}} \\
-\frac{\mathrm{i} N}{4} p_{1} & \mathrm{e}^{-\frac{\mathrm{i} N}{4} p_{1}} & \mathrm{e}^{-\frac{\mathrm{i} N}{4} p_{1} \lambda_{1}} & \ldots & \mathrm{e}^{-\frac{\mathrm{i} N}{4} p_{1} \lambda_{N}} & \mathrm{e}^{-\frac{\mathrm{i} N}{4} p_{1}} \\
\vdots & \vdots & & \vdots & \vdots \\
-\frac{\mathrm{i} N}{4} p_{N} & \mathrm{e}^{-\frac{\mathrm{i} N}{4} p_{N}} & \mathrm{e}^{-\frac{\mathrm{i} N}{4} p_{N} \lambda_{1}} & \ldots & \mathrm{e}^{-\frac{\mathrm{i} N}{4} p_{N} \lambda_{N}} & \mathrm{e}^{-\frac{\mathrm{i} N}{4} p_{N}}
\end{array}\right| \\
& =\sum_{\pi_{+} \in \mathfrak{S}_{N}} \operatorname{sgn}\left(\pi_{+}\right)\left[\left(1-\frac{\mathrm{i} N}{4} p_{1}\right) \mathrm{e}^{-\frac{\mathrm{i} N}{4} p_{1}} \prod_{i=1}^{N} \mathrm{e}^{-\frac{\mathrm{i} N}{4} \lambda_{i} p_{\pi_{+}(i)}}\right. \\
& \left.-\frac{\mathrm{i} N}{4} \sum_{i=1}^{N} \lambda_{i} \mathrm{e}^{-\frac{\mathrm{i} N}{4} \lambda_{i} p_{1}} p_{\pi_{+}(i)} \mathrm{e}^{-\frac{\mathrm{i} N}{4} p_{\pi_{+}(i)}} \prod_{\substack{k=1 \\
k \neq i}}^{N} \mathrm{e}^{-\frac{\mathrm{i} N}{4} \lambda_{k} p_{\pi_{+}}(k)}\right] .
\end{aligned}
$$

When inserted into the expression (7.20), we can use the invariance of the radial integration measure and domain under permutations of the $\lambda_{i}$ 's to reduce the double sum over the Weyl groups to a single sum over the relative permutation $\pi:=\pi_{+} \pi_{-}^{-1} \in \mathfrak{S}_{N}$ with $\pi(N)=\pi_{+}(N)$. The sum over $\pi_{+}$can be replaced by a sum over $\pi$, while the remaining sum over $\pi_{-}$simply produces the order $N$ ! of the Weyl group of $U(N)$.

In this way we may bring the Fourier transform of the orbit into the form

$$
\begin{gathered}
Z_{\mathcal{O}(p)=}-\frac{4 \operatorname{vol}(\mathcal{O})}{p_{1} \Delta(p)^{2}} \frac{\prod_{k=1}^{N}(k !)^{2}}{(N-1) !(\sqrt{8} N)^{N^{2}-N}} \\
\times \sum_{\pi \in \mathfrak{S}_{N}} \operatorname{sgn}(\pi)\left[\left(1-\frac{\mathrm{i} N}{4} p_{1}\right) \mathrm{e}^{-\frac{\mathrm{i} N}{4}\left(p_{1}+p_{\pi(N)}\right)} \prod_{i=1}^{N_{-}} \int_{-1}^{1} \mathrm{~d} \lambda_{i} \mathrm{e}^{-\frac{\mathrm{i} N}{4} \lambda_{i}\left(p_{\pi(i)}-p_{i+1}\right)}\right. \\
\quad-\frac{\mathrm{i} N}{4} \sum_{j=1}^{N_{-}} p_{\pi(j)} \mathrm{e}^{-\frac{\mathrm{i} N}{4}\left(p_{\pi(j)}+p_{\pi(N)}\right)} \\
\quad \times \int_{-1}^{1} \mathrm{~d} \lambda_{j} \lambda_{j} \mathrm{e}^{-\frac{\mathrm{i} N}{4} \lambda_{j}\left(p_{1}-p_{j+1}\right)} \prod_{\substack{i=1 \\
i \neq j}}^{N_{-}} \int_{-1}^{1} \mathrm{~d} \lambda_{i} \mathrm{e}^{-\frac{\mathrm{i} N}{4} \lambda_{i}\left(p_{\pi(i)}-p_{i+1}\right)} \\
\left.-\frac{\mathrm{i} N}{4} p_{\pi(N)} \mathrm{e}^{-\frac{\mathrm{i} N}{4}\left(p_{\pi(N)}+p_{1}\right)} \prod_{i=1}^{N_{-}} \int_{-1}^{1} \mathrm{~d} \lambda_{i} \mathrm{e}^{-\frac{\mathrm{i} N}{4} \lambda_{i}\left(p_{\pi(i)}-p_{i+1}\right)}\right]
\end{gathered}
$$

Finally, the radial integrations can be expressed in terms of the spectral sine-kernel of the unitary ensemble of random matrix theory and its derivative given by

$$
\mathrm{K}(x):=\frac{\sin x}{x}=\frac{1}{2} \int_{-1}^{1} \mathrm{~d} \lambda \mathrm{e}^{-\mathrm{i} \lambda x} \quad \text { and } \quad \mathrm{K}^{\prime}(x)=\frac{1}{x}\left(\cos x-\frac{\sin x}{x}\right)=-\frac{\mathrm{i}}{2} \int_{-1}^{1} \mathrm{~d} \lambda \lambda \mathrm{e}^{-\mathrm{i} \lambda x} .
$$

Then the abelianized partition function (5.2) is written as an exact expansion in gaussian momen- 
tum transforms given by

$$
\begin{aligned}
& Z=-\frac{8 \operatorname{vol}(\mathcal{O}) N !(N-1) ! \prod_{k=1}^{N-2}(k !)^{2}}{2^{N^{2}}(2 \pi)^{N}(\sqrt{2} N)^{N^{2}-N}} \sum_{\pi \in \mathfrak{S}_{N}} \operatorname{sgn}(\pi) \int_{\mathbb{R}^{N}}[\mathrm{~d} p] \frac{\mathrm{e}^{-\frac{g}{4 N} \sum_{i} p_{i}^{2}}}{p_{1}} \\
& \times\left[\left(1-\frac{\mathrm{i} N}{4} p_{1}\right) \mathrm{e}^{-\frac{\mathrm{i} N}{4}\left(p_{1}+p_{\pi(N)}\right)} \prod_{i=1}^{N_{-}} \mathrm{K}\left(\frac{N}{4}\left(p_{\pi(i)}-p_{i+1}\right)\right)\right. \\
& +\frac{N}{4} \sum_{j=1}^{N_{-}} p_{\pi(j)} \mathrm{e}^{-\frac{\mathrm{i} N}{4}\left(p_{\pi(j)}+p_{\pi(N)}\right)} \mathrm{K}^{\prime}\left(\frac{N}{4}\left(p_{1}-p_{j+1}\right)\right) \prod_{\substack{i=1 \\
i \neq j}}^{N_{-}} \mathrm{K}\left(\frac{N}{4}\left(p_{\pi(i)}-p_{i+1}\right)\right) \\
& \left.-\frac{\mathrm{i} N}{4} p_{\pi(N)} \mathrm{e}^{-\frac{\mathrm{i} N}{4}\left(p_{1}+p_{\pi(N)}\right)} \prod_{i=1}^{N_{-}} \mathrm{K}\left(\frac{N}{4}\left(p_{\pi(i)}-p_{i+1}\right)\right)\right] .
\end{aligned}
$$

For low values of $N$, the momentum integrals in this formula can be computed in terms of transcendental error functions, which are the typical contributions in nonabelian localization [18] and reflect the occurence of non-gaussian quantum fluctuation integrals. Note that there is a single momentum $p_{1}$ singled out in the formula (7.24). In the $U(n)$ case of Section 7.3 below there will be $n$ momenta singled out which is where the sum over sets of $n$ integers required by the nonabelian localization formula in the large $N$ limit will come from. At $N \rightarrow \infty$, the spectral kernels $\mathrm{K}\left(\frac{N}{4}\left(p_{\pi(i)}-p_{i+1}\right)\right) \approx \frac{4 \pi}{N} \delta\left(p_{\pi(i)}-p_{i+1}\right)$ provide the necessary groupings of variables into partitions of $N$ arising from the sum over the residual gauge symmetry group $\mathfrak{S}_{N}$. The conjugacy class of a given permutation $\pi \in \mathfrak{S}_{N}$ is characterized entirely by its cycle decomposition, which contains $n_{k} \geq 0$ cycles of length $k$ for $k=1, \ldots, N$ with $N=\sum_{k} k n_{k}$ and $\operatorname{sgn}(\pi)=(-1)^{\sum_{k}(k-1) n_{k}}$. However, the saddle-point partitions here do not correspond to the cycles themselves, but rather to the numbers $N_{n_{1}, \ldots, n_{N}}$ of cycles $\left(n_{1}, \ldots, n_{N}\right)$. For instance, the vacuum state now corresponds to the instanton configuration with $N$ fluxons, i.e. only trivial representations due to the abelianization, with moduli space (3.11) as described in Section 3.1. The higher critical points consist of an even number of irreducible representations which are suppressed roughly as $\mathrm{e}^{-N^{3} / 2 g n_{i}}$. This indicates that the radial coordinates on the configuration space $\mathcal{O}$ are not so nicely adapted to the local symplectic geometry of the Yang-Mills critical surfaces. We will return to these issues in the next section.

\subsection{Evaluation of the abelianized partition function: $U(n)$ gauge theory}

The nonabelian case $n>1$ becomes very complicated due to the increasing complexity of the combinatorics involved in regulating the Itzykson-Zuber integral (6.1) over $V_{+} \in U\left(n N_{+}\right)$. We will therefore only briefly sketch the essential features, defering the explicit evaluation in favour of a more formal, regulated combinatorial expansion. Consider the radial coordinates $\lambda_{i}, i=1, \ldots, n N_{-}$ on $\mathcal{O}$ and add $2 n$ new real variables $1+\varepsilon_{i}$. We assemble them into the ordered set defined by

$$
\left(\bar{\lambda}_{1}, \ldots, \bar{\lambda}_{n N_{+}}\right):=\left(1+\varepsilon_{1}, \ldots, 1+\varepsilon_{2 n}, \lambda_{1}, \ldots, \lambda_{n N_{-}}\right) .
$$

Similarly, we double the first $n$ entries of the momentum vector $p=\left(p_{1}, \ldots, p_{n N}\right)$ and gather them into the ordered set defined by

$$
\left(\bar{p}_{1}, \ldots, \bar{p}_{n N_{+}}\right):=\left(p_{1}+\kappa, \ldots, p_{n}+\kappa, p_{1}, \ldots, p_{n}, p_{n+1}, \ldots, p_{n N}\right) .
$$

At the end we will take the limits $\varepsilon_{i}, \kappa \rightarrow 0$. 
The evaluation of the Fourier transform (5.3) now proceeds exactly as in Section 7.2 above. To organize the combinatorics, we use the identity

$$
\begin{aligned}
& \lim _{\varepsilon_{i} \rightarrow 0} \frac{\operatorname{det}_{1 \leq i, j \leq n N_{+}}\left(\mathrm{e}^{-\frac{\mathrm{i} N}{4} \bar{p}_{i} \bar{\lambda}_{j}}\right)}{\Delta(\varepsilon)} \\
& \quad=\frac{\operatorname{vol}(U(2 n))}{c_{N}(2 n,-4)} \sum_{\mathcal{Q} \subset\left\{\bar{p}_{i}\right\}} \operatorname{sgn}\left(\mathcal{Q} \hookrightarrow\left\{\bar{p}_{i}\right\}\right) \mathrm{e}^{-\frac{\mathrm{i} N}{4} \sum_{i} q_{i}} \Delta(q) \operatorname{det}_{1 \leq i, j \leq n N_{-}}\left(\mathrm{e}^{-\frac{\mathrm{i} N}{4} \hat{p}_{i} \lambda_{j}}\right)
\end{aligned}
$$

where $\left\{\hat{p}_{1}, \ldots, \hat{p}_{n N_{-}}\right\}=\left\{\bar{p}_{1}, \ldots, \bar{p}_{n N_{+}}\right\} \backslash \mathcal{Q}$ with $\mathcal{Q}=\left\{q_{1}, \ldots, q_{2 n}\right\}$ a subset of $\left\{\bar{p}_{1}, \ldots, \bar{p}_{n N_{+}}\right\}$ which is ordered according to (7.26), and the sign is determined by the parity of the embedding. The identity (7.27) can be derived by performing a Laplace expansion of the determinant on the left-hand side into the $2 n$ rows containing the variables $1+\varepsilon_{i}$, and using the limit formula

$$
\lim _{\varepsilon_{i} \rightarrow 0} \frac{\operatorname{det}_{1 \leq i, j \leq 2 n}\left(\mathrm{e}^{-\frac{\mathrm{i} N}{4} q_{i} \varepsilon_{j}}\right)}{\Delta(\varepsilon)}=\frac{\operatorname{vol}(U(2 n))}{c_{N}(2 n,-4)} \Delta(q)
$$

which follows from the Itzykson-Zuber formula (6.1). The Vandermonde determinants can also be factorized as

$$
\Delta(\bar{\lambda})=\Delta(\lambda) \Delta(\varepsilon) \prod_{i=1}^{n N_{-}}\left(1-\lambda_{i}\right)^{2 n}
$$

up to higher order terms in $\varepsilon_{i} \rightarrow 0$, along with

$$
\Delta(\bar{p}) \Delta\left(p_{n+1}, \ldots, p_{n N}\right)=\kappa^{n} \Delta(p)^{2} \Delta\left(p_{1}, \ldots, p_{n}\right)^{2}
$$

in the limit $\kappa \rightarrow 0$.

In this way the partition function (5.2) can be expanded as

$$
\begin{aligned}
& Z=\zeta_{n, N} \lim _{\kappa \rightarrow 0} \frac{1}{\kappa^{n}} \sum_{\mathcal{Q} \subset\left\{\bar{p}_{i}\right\}} \operatorname{sgn}\left(\mathcal{Q} \hookrightarrow\left\{\bar{p}_{i}\right\}\right) \int_{\mathbb{R}^{n N}}[\mathrm{~d} p] \frac{\mathrm{e}^{-\frac{g}{4 N} \sum_{i} p_{i}^{2}}}{\Delta\left(p_{1}, \ldots, p_{n}\right)^{2}} \mathrm{e}^{-\frac{\mathrm{i} N}{4} \sum_{i} q_{i}} \Delta(q) \\
& \times \prod_{l=1}^{n N_{-}} \int_{-1}^{1} \mathrm{~d} \lambda_{l} \operatorname{det}_{1 \leq i, j \leq n N_{-}}\left(\mathrm{e}^{\frac{\mathrm{i} N}{4} p_{i+n} \lambda_{j}}\right) \operatorname{det}_{1 \leq i, j \leq n N_{-}}\left(\mathrm{e}^{-\frac{\mathrm{i} N}{4} \hat{p}_{i} \lambda_{j}}\right)
\end{aligned}
$$

where

$$
\zeta_{n, N}:=\frac{\operatorname{vol}(\mathcal{O})}{(n N) !(2 \pi)^{n N}} \frac{(\mathrm{i} N)^{2 n^{2}+n N\left(1-n N_{+}\right)}}{2^{n N_{-}\left(2-n N_{+}\right)}} \prod_{k=1}^{n N_{-}-1}(k !)^{2} \prod_{m=1}^{2 n} \frac{\left(m+n N_{-}-1\right) !}{m !} .
$$

We now expand the two determinants in (7.31) into a double sum over the Weyl group $\mathfrak{S}_{n} N_{-}$, and use permutation symmetry of the radial integration to rewrite it as a sum over a single relative permutation exactly as in Section 7.2 above. Using (7.23) we arrive finally at the exact combinatorial expansion

$$
\begin{aligned}
Z= & 2^{n N_{-}}\left(n N_{-}\right) ! \zeta_{n, N} \lim _{\kappa \rightarrow 0} \frac{1}{\kappa^{n}} \sum_{\mathcal{Q} \subset\left\{\bar{p}_{i}\right\}} \operatorname{sgn}\left(\mathcal{Q} \hookrightarrow\left\{\bar{p}_{i}\right\}\right) \sum_{\pi \in \mathfrak{S}_{n} N_{-}} \operatorname{sgn}(\pi) \\
& \times \int_{\mathbb{R}^{n N}}[\mathrm{~d} p] \frac{\mathrm{e}^{-\frac{g}{4 N} \sum_{i} p_{i}^{2}}}{\Delta\left(p_{1}, \ldots, p_{n}\right)^{2}} \mathrm{e}^{-\frac{\mathrm{i} N}{4} \sum_{i} q_{i}} \Delta(q) \prod_{i=1}^{n N_{-}} \mathrm{K}\left(\frac{N}{4}\left(\hat{p}_{\pi(i)}-p_{i+n}\right)\right) .
\end{aligned}
$$


The combinatorics of the large $N$ limit of the partition function (7.33) can be described as follows. The sine-kernels $\mathrm{K}\left(\frac{N}{4}\left(\hat{p}_{\pi(i)}-p_{i+n}\right)\right) \approx \frac{4 \pi}{N} \delta\left(\hat{p}_{\pi(i)}-p_{i+n}\right)$ define a link from $\hat{p}_{\pi(i)}$ to $p_{i+n}$. Following these, we obtain a set of open or closed links determined by $\pi \in \mathfrak{S}_{n N_{-}}$. The open links must start at $\left\{p_{1}+\kappa, \ldots, p_{n}+\kappa, p_{1}, \ldots, p_{n}\right\}$ (since those are not contained in the $p_{i+n}$ ) and end at $\left\{q_{1}, \ldots, q_{2 n}\right\}$ (since those are not contained in the $\hat{p}_{i}$ ). The closed links correspond to cycles in the conjugacy class of the permutation $\pi$. In particular, there are no factors $\mathrm{e}^{-\frac{\mathrm{i} N}{4} p_{i}}, i=1, \ldots, n$ or $\Delta\left(p_{1}, \ldots, p_{n}\right)^{2}$ for the internal variables, and hence we can explicitly evaluate the internal integrals. The difficulty lies in evaluating the sum over all possible distinct cycles for the internal variables in a closed form.

\section{Comparison with the constrained matrix model}

In [9], quantum gauge theory on the fuzzy sphere $S_{N}^{2}$ was formulated as a multi-matrix model with action

$$
S_{\mathrm{mm}}=\frac{1}{N g} \operatorname{Tr}\left(C^{2}-\frac{N^{2}}{4} \mathbb{1}_{\mathcal{N}}\right)^{2}
$$

and the constraint $C_{0}=\frac{1}{2} \mathbb{1}_{N}$. It was shown that this matrix model also reproduces Yang-Mills theory on $S^{2}$ in the large $N$ limit. This differs from the formulation of the present paper essentially by replacing the pair (action, constraint) given by $\left(\left(C^{2}-\frac{N^{2}}{4} \mathbb{1}_{\mathcal{N}}\right)^{2},\left(C_{0}-\frac{1}{2} \mathbb{1}_{N}\right)\right)$ with the permuted pair $\left(\left(C_{0}-\frac{1}{2} \mathbb{1}_{N}\right)^{2},\left(C^{2}-\frac{N^{2}}{4} \mathbb{1}_{\mathcal{N}}\right)\right)$. This can be understood by imposing the respective constraints using gaussian terms in the actions, as then the tangential degrees of freedom are essentially the same in both cases. The symplectic formulation of the present paper has not only the advantage of applying the equivariant localization principle to systematically construct the instanton expansion of gauge theory on the fuzzy sphere, but it also somewhat simplifies the evaluation of the matrix integral. It also enables one in principle to keep control of the $\frac{1}{N}$ corrections to Yang-Mills theory on $S^{2}$, and the approximate delta-functions at $N \rightarrow \infty$ responsible for the groupings of variables are more transparent along the lines explained in Sections 7.2 and 7.3.

\section{Yang-Mills critical surfaces in abelianized localization}

In this final section we will elucidate the relationship between the nonabelian and abelianized localization approaches to the exact instanton expansion of Yang-Mills theory on the fuzzy sphere $S_{N}^{2}$. As discussed above, the critical surfaces for abelian localization are determined by the saddlepoint equation (6.4), (7.2)

$$
[C, \Phi]=0
$$

for $\Phi=\phi \otimes \sigma^{0}$ with $\phi \in \mathfrak{u}(N)$, which can be assumed to be diagonal by using a gauge transformation. Its distinct eigenvalues $\Phi_{\nu}$ are arranged into degenerate blocks as

$$
\Phi=\bigoplus_{\nu=1}^{k} \Phi_{\nu} \mathbb{1}_{n_{\nu}} \otimes \sigma^{0}
$$

with $\sum_{\nu} n_{\nu}=N$. Then $[C, \Phi]=0$ implies that the covariant coordinate

$$
C=U^{-1} \Xi U=\bigoplus_{\nu=1}^{k} C_{\nu}
$$

has the same block decomposition as $\Phi$. Thus it can be diagonalized as

$$
C_{\nu}=V_{\nu}^{-1} \Xi_{\nu} V_{\nu}
$$


where $V_{\nu}$ is a $2 n_{\nu} \times 2 n_{\nu}$ unitary matrix on the block defined by $\mathbb{1}_{n_{\nu}} \otimes \sigma^{0}$ in (8.2), and $\Xi_{\nu}$ has eigenvalues $\pm \frac{N}{2}$. Then comparing (8.3) and (8.4) implies

$$
\left(\bigoplus_{\nu=1}^{k} V_{\nu}\right) U^{-1} \Xi U\left(\bigoplus_{\nu=1}^{k} V_{\nu}^{-1}\right)=\bigoplus_{\nu=1}^{k} \Xi_{\nu}=\Sigma^{-1} \Xi \Sigma
$$

for some permutation matrix $\Sigma \in U(\mathcal{N})$ representing an element $\pi \in \mathfrak{S}_{\mathcal{N}} / \mathfrak{S}_{N_{+}} \times \mathfrak{S}_{N_{-}}$, since both $\Xi$ and $\bigoplus_{\nu} \Xi_{\nu}$ are diagonal $\mathcal{N} \times \mathcal{N}$ matrices with the same set of degenerate eigenvalues. It follows that

$$
U\left(\bigoplus_{\nu=1}^{k} V_{\nu}^{-1}\right) \Sigma^{-1} \in U\left(N_{+}\right) \times U\left(N_{-}\right)
$$

and therefore $U \in U(\mathcal{N})$ is equal to $\Sigma\left(\bigoplus_{\nu} V_{\nu}\right)$ times an element of the stablizer subgroup $U\left(N_{+}\right) \times$ $U\left(N_{-}\right) \subset U(\mathcal{N})$ of the element $\Xi$.

We conclude that the gauge equivalence classes of solutions of the saddle point equation $[C, \Phi]=$ 0 in the configuration space $\mathcal{O}$ are described by the following data:

- A quotient permutation $\pi \in \mathfrak{S}_{\mathcal{N}} / \mathfrak{S}_{N_{+}} \times \mathfrak{S}_{N_{-}}$;

- A unitary matrix in the stabilizer group $U\left(N_{+}\right) \times U\left(N_{-}\right)$; and

- A unitary block transformation $\bigoplus_{\nu} V_{\nu}$ adapted to the block decomposition (8.2) of $\Phi$.

It is evident that these critical surfaces are much larger than the critical surfaces of the original Yang-Mills action, and they are not even in any one-to-one correspondence with the Yang-Mills saddle points. Any such block configuration is degenerate for the action in (4.3), and contains some Yang-Mills blocks of Section 3.4 (with the irreducible low-energy critical surface $\mathcal{C}_{(N, 1)}$ and possibly fluxons or other purely noncommutative solutions). The reason is the absence of any localization form $Q \alpha$, without which there is no way to separate the desired Yang-Mills blocks of Section 3.4 from these abelianized critical surfaces.

\subsection{Itzykson-Zuber localization on the symplectic leaves}

We now consider the foliation of the orbit $\mathcal{O}(\Xi) \cong U(2 N) / R$ by conjugacy classes under the adjoint action of the stabilizer group $R=U\left(N_{+}\right) \times U\left(N_{-}\right)$. The corresponding symplectic leaves $\mathcal{L}(\lambda)$ are parametrized by the radial coordinates $\lambda_{i} \in[-1,1], i=1, \ldots, N_{-}$. For a given leaf $\mathcal{L}(\lambda)$, the integral $\int_{R}[\mathrm{~d} V] \mathrm{e}^{-\frac{\mathrm{i}}{2}\left\langle\mu_{T}(C), p\right\rangle}$ is obtained by using the Itzykson-Zuber formula for the unitary groups $U\left(N_{+}\right)$and $U\left(N_{-}\right)$, as we did in Sections 7.2 and 7.3. As in Section 6 above, the ItzyksonZuber formula can itself be regarded as a consequence of abelian localization, and the expansions of the resulting determinants in Section 7.2 is precisely the sum over the saddle-points on each leaf $\mathcal{L}(\lambda)$.

Let us identify these saddle-points explicitly. Choosing $\Xi$ as in (7.13), the critical points of the moment map (7.15) with respect to arbitrary variations of $\left(V_{+}, V_{-}\right) \in R$ are given by the solutions of the equations

$$
\begin{aligned}
{\left[\operatorname{diag}\left(p_{2}, \ldots, p_{N}\right), V_{-} \operatorname{diag}\left(\lambda_{1}, \ldots, \lambda_{N_{-}}\right) V_{-}^{-1}\right] } & =0 \\
{\left[\operatorname{diag}\left(p_{1} \sigma^{0}, p_{2}, \ldots, p_{N}\right), V_{+} \operatorname{diag}\left(\sigma^{0}, \lambda_{1}, \ldots, \lambda_{N_{-}}\right) V_{+}^{-1}\right] } & =0 .
\end{aligned}
$$

As in Section 7.3, we consider for convenience the extended sets of radial coordinates (7.25) and momentum variables (7.26) for $n=1$. Then the first equation in (8.7) means that the matrix 
$V_{-} \operatorname{diag}\left(\lambda_{1}, \ldots, \lambda_{N_{-}}\right) V_{-}^{-1}$ commutes with the spectral projectors of $\left(p_{2}, \ldots, p_{N}\right)$, i.e. it has the same block decomposition, and similarly the second equation in (8.7) implies that the matrix $V_{+} \operatorname{diag}\left(\bar{\lambda}_{1}, \ldots, \bar{\lambda}_{N_{+}}\right) V_{+}^{-1}$ commutes with the spectral projectors of $\bar{p}$.

Using unitary transformations on each of these blocks, the matrix $V_{-} \operatorname{diag}\left(\lambda_{1}, \ldots, \lambda_{N_{-}}\right) V_{-}^{-1}$ can then be diagonalized with the same eigenvalues $\lambda_{i}$. It follows that

$$
\begin{aligned}
&\left(\bigoplus_{\nu=1}^{k} U_{\nu}\right) V_{-} \operatorname{diag}\left(\lambda_{1}, \ldots, \lambda_{N_{-}}\right) V_{-}^{-1}\left(\bigoplus_{\nu=1}^{k} U_{\nu}^{-1}\right) \\
&=\operatorname{diag}\left(\lambda_{\pi_{-}(1)}, \ldots, \lambda_{\pi_{-}\left(N_{-}\right)}\right)=\Sigma_{-} \operatorname{diag}\left(\lambda_{1}, \ldots, \lambda_{N_{-}}\right) \Sigma_{-}^{-1}
\end{aligned}
$$

for some $U_{\nu} \in S U\left(n_{\nu}\right)$, where $n_{\nu}$ labels the degenerate blocks of $\left(p_{2}, \ldots, p_{N}\right)$ with $\sum_{\nu} n_{\nu}=N_{-}$ and $\Sigma_{-} \in S U\left(N_{-}\right)$is a permutation matrix corresponding to an element $\pi_{-} \in \mathfrak{S}_{N_{-}}$. If $\lambda_{i}$ are nondegenerate, this implies that $\left(\bigoplus_{\nu} U_{\nu}\right) V_{-}=\Sigma_{-}$and hence

$$
V_{-}=\left(\bigoplus_{\nu=1}^{k} U_{\nu}^{-1}\right) \Sigma_{-}
$$

If some $\lambda_{i}$ are degenerate, it only follows that $\Sigma_{-}^{-1}\left(\bigoplus_{\nu} U_{\nu}\right) V_{-}$commutes with the spectral projectors of $\lambda$, so that $\Sigma_{-}^{-1}\left(\bigoplus_{\nu} U_{\nu}\right) V_{-}=\bigoplus_{\nu} \tilde{U}_{\nu}$ for some $\tilde{U}_{\nu} \in S U\left(n_{\nu}\right)$. It follows that the angular saddle-point $V_{-} \in U\left(N_{-}\right)$is given by

$$
V_{-}=\left(\bigoplus_{\nu=1}^{k} U_{\nu}^{-1}\right) \Sigma_{-}\left(\bigoplus_{\nu=1}^{k} \tilde{U}_{\nu}\right)
$$

Similar statements hold for the angular saddle-point $V_{+} \in U\left(N_{+}\right)$, with the additional feature that the first two entries of $\bar{p}$ and $\bar{\lambda}$ are degenerate by definition.

In each case, the value of the action (7.15) is given by

$$
\left\langle\mu_{T}(C), p\right\rangle=\frac{N}{4} \sum_{i=1}^{N_{+}} \bar{p}_{i} \bar{\lambda}_{\pi_{+}(i)}-\frac{N}{4} \sum_{i=1}^{N_{-}} p_{i+1} \lambda_{\pi_{-}(i)} .
$$

Therefore, each saddle-point is characterized by two permutation matrices $\Sigma_{ \pm}$corresponding to $\pi_{ \pm} \in \mathfrak{S}_{N_{ \pm}}$, which may or may not generate non-trivial fibers on the homogeneous spaces of the group $\prod_{\nu} U\left(n_{\nu}\right)$ depending on the degeneracies of $p$ and $\lambda$. The integral over these $V_{ \pm}$orbits can then be evaluated using the Itzykson-Zuber formula leading to (7.16) and (7.17), which gives precisely the sum over the saddle points. The regularization required in (7.17) reflects the fact that the critical surfaces are no longer isolated points, due to the degeneracies of $\bar{\lambda}_{i}$ and $\bar{p}_{i}$.

The main point of this analysis is that these critical surfaces are again not in any one-to-one correspondence with those of the original Yang-Mills action. In fact, the abelian critical surfaces above contain as subspaces those of the Itzykson-Zuber localization on $\mathcal{O}(\Xi)$ discussed in Section 6 above, which are not only stationary on the symplectic leaves $\mathcal{L}(\lambda)$ but also with respect to variations of the radial coordinates $\lambda_{i}$. However, even the critical surfaces for the Itzykson-Zuber localization on the configuration space $\mathcal{O}(\Xi)$ are not simply related to those of the Yang-Mills action. In particular, the variational problem for the action (8.11) does not determine the $\lambda_{i}$. A given radial saddle-point $\pi_{ \pm}$can thus correspond to various types of Yang-Mills solutions by appropriately choosing some $\lambda_{i}$, as we show explicitly in Section 8.2 below. This arbitrariness in the radial coordinates $\lambda_{i}$ is lifted by the addition of the localization one-form $\alpha$ of Section 4 , which serves to single out the Yang-Mills saddle points from the new critical points. Nevertheless, it is instructive to work out the radial coordinates of some Yang-Mills saddle-points to illustrate the powerful workings of the polar decomposition. 


\subsection{Radial coordinates for Yang-Mills critical surfaces}

We will now work out the radial coordinates for the solutions of the Yang-Mills equations $\left[C_{0}, C_{i}\right]=$ 0 , which will identify precisely the appropriate localization values of $\lambda_{i}$ for each critical surface of Section 3.1. Given (7.13) we now consider the fuzzy sphere coordinates $\Sigma_{0} \Xi \Sigma_{0}^{-1}$ and correspondingly modify the radial coordinates (7.12) to

$$
R=\Sigma_{0}\left(\begin{array}{cc}
\sigma^{0} & 0 \\
0 & \exp \left(\mathrm{i} \sigma^{1} \otimes \rho\right)
\end{array}\right) \Sigma_{0}^{-1}
$$

where $\Sigma_{0} \in U(\mathcal{N})$ is a permutation matrix representing the cyclic permutation

$$
\pi_{\left(N_{+}\right)}=\left(12 \cdots N_{+}\right) .
$$

As we will see, the modification by $\Sigma_{0}$, although irrelevant from the point of view of the path integral, will greatly simplify the explicit parametrization.

Using this parametrization and (7.14), we can write the covariant coordinates (7.5) in the explicit form

$$
\begin{aligned}
C & =\frac{N}{2} V \Sigma_{0}\left(\begin{array}{ccc}
\sigma^{0} & & 0 \\
0 & \sigma^{3} \otimes \cos (2 \rho)+\sigma^{2} \otimes \sin (2 \rho)
\end{array}\right) \Sigma_{0}^{-1} V^{-1} \\
& =\frac{N}{2}\left(\begin{array}{cc}
V_{+}\left(\begin{array}{ccc}
1 & & \\
& \cos (2 \rho) & \\
& & 1
\end{array}\right) V_{+}^{-1} & -\mathrm{i} V_{+}\left(\begin{array}{c}
0 \\
\sin (2 \rho) \\
0
\end{array}\right) V_{-}^{-1} \\
i V_{-}\left(\begin{array}{lll}
0, & \sin (2 \rho), & 0
\end{array}\right) V_{+}^{-1} & -V_{-} \cos (2 \rho) V_{-}^{-1}
\end{array}\right)
\end{aligned}
$$

where we have applied the commutation relation $\mathrm{i}\left[\sigma^{1}, \sigma^{3}\right]=2 \sigma^{2}$. The role of the cyclic permutation matrix $\Sigma_{0}$ is to move the unit entries of $\sigma^{0}$ symmetrically around the matrix $\cos (2 \rho)$. We note for later use that if the unitary matrices $V_{ \pm} \in U\left(N_{ \pm}\right)$are block-diagonal, then so is $C$. We will now use this parametrization to illustrate the use of the radial coordinates by working out (8.14) explicitly for various classical gauge field configurations of Section 3.1.

\section{The vacuum solution}

The generators of the irreducible $N$-dimensional representation of the $\mathfrak{s u}(2)$ Lie algebra (2.1) are given explicitly by

$$
\left(\xi_{3}\right)_{i j}=-\delta_{i j} \frac{N+1-2 i}{2} \quad \text { and } \quad\left(\xi_{+}\right)_{i j}=\delta_{i+1, j} \sqrt{(N-i) i}
$$

where $i, j=1, \ldots, N$ and $\xi_{ \pm}=\xi_{1} \pm \mathrm{i} \xi_{2}$ with $\xi_{-}=\xi_{+}^{\dagger}$. The vacuum solution (2.4) in the abelian case $n=1$ thus has the explicit form

$$
\begin{aligned}
C & =\left(\begin{array}{cc}
\frac{1}{2} \mathbb{1}_{N}+\xi_{3} & \xi_{+} \\
\xi_{-} & \frac{1}{2} \mathbb{1}_{N}-\xi_{3}
\end{array}\right) \\
& =\left(\begin{array}{cc}
\frac{1}{2} \operatorname{diag}(-N+2, \ldots, N-2, N) & \xi_{+} \\
\xi_{-} & \frac{1}{2} \operatorname{diag}(N, \ldots,-N+4,-N+2)
\end{array}\right)
\end{aligned}
$$

using the splitting into equal blocks of size $N$. This should be identified with (8.14), which splits into blocks of sizes $N_{ \pm}$. Noting the explicit form of $\xi_{ \pm}$in (8.15) as raising and lowering operators, it follows that one can consistently take both $V_{+} \operatorname{diag}\left(\lambda_{1}, \ldots, \lambda_{N_{-}}, 1,1\right) V_{+}^{-1}$ and $V_{-} \operatorname{diag}\left(\lambda_{1}, \ldots, \lambda_{N_{-}}\right) V_{-}^{-1}$ to be diagonal matrices. 
We can then consistently match the eigenvalues as

$$
N\left(\lambda_{1}, \ldots, \lambda_{N_{-}}, 1,1\right)=(-N+2, \ldots, N-2, N, N),
$$

which gives

$$
\lambda_{i}=-\frac{N-2 i}{N} \quad \text { for } \quad i=1, \ldots, N_{-}
$$

and provides the eigenvalues of the radial matrix $R$ for the vacuum critical surface $\mathcal{C}_{(N, 1)}$. Note that the eigenvalue $\frac{N}{2}$ from the second diagonal block $\frac{1}{2} \mathbb{1}_{N}-\xi_{3}$ of $C$ in (8.16) is contained in the matrix $\frac{N}{2} V_{+} \operatorname{diag}\left(\lambda_{1}, \ldots, \lambda_{N_{-}}, 1,1\right) V_{+}^{-1}$. It follows that $V_{-}=\Sigma_{-}$is a permutation matrix in $U\left(N_{-}\right)$, while $V_{+}=\Sigma_{+} U_{2}$ is a permutation matrix up to a possible conjugation with a unitary matrix $U_{2} \in S U(2) \subset U\left(N_{+}\right)$acting on the two marked indices labelling the unit entries. We can absorb $\Sigma_{-}$by a redefinition of the $\lambda_{i}$, and hence take

$$
V_{-}=\mathbb{1}_{N_{-}}
$$

without loss of generality. It is also enough to consider the case $U_{2}=\mathbb{1}_{N_{+}}$. Comparing (8.14) with (8.16), it follows that

$$
V_{+}=\Sigma_{+}
$$

is a permutation matrix representing the irreducible cycle (8.13) of length $N_{+}$. Furthermore, one has

$$
\sin \left(2 \rho_{i}\right)=\sqrt{1-\lambda_{i}^{2}}=\sqrt{\frac{4 i}{N}-\frac{4 i^{2}}{N^{2}}}=\frac{2}{N} \sqrt{i(N-i)}=\frac{2}{N}\left(\xi_{+}\right)_{i, i+1}
$$

for $i=1, \ldots, N_{-}$, which is indeed the correct representation of $\xi_{ \pm}$in (8.15), embedded in the correct off-diagonal way in (8.14) due to the block decomposition into sizes $N_{ \pm}$.

Let us point out one interesting feature of the covariant coordinate (8.16). The two diagonal entries of $\frac{N}{2}$ in the center of the matrix constitute a trivial $2 \times 2$ unit matrix $\sigma^{0}$ which completely decouples from the rest of $C$. This block can be traced to the $\sigma^{0}$ in the upper-left corner of the first line in (8.14), whose position is determined by the permutation matrix $\Sigma_{0}$, or equivalently to the auxilliary radial coordinates $\bar{\lambda}_{i}=1+\varepsilon_{i}, i=1,2$. In fact, any explicit entry of $\pm \frac{N}{2}$ in $C$ necessarily decouples from the rest of $C$, for otherwise $C$ would have eigenvalues of modulus larger than $\frac{N}{2}$. This means, in particular, that we can permute these two entries using a suitable permutation matrix $V_{+}=\Sigma_{+}$without any effect on $C$ (but it will have an effect on the momenta $p_{i}$ if they are included). This observation will be useful below. This construction clearly generalizes to give the blocks $C\left(n_{a}\right)$ of size $2 n_{a}$ of the critical surfaces $\mathcal{C}_{\left(n_{1}, s_{1}\right), \ldots,\left(n_{k}, s_{k}\right)}$ corresponding to irreducible $S U(2)$ representations of dimensions $n_{a}<N$. The most extreme case $n_{a}=1$ consists of the one-dimensional representation with $C_{0}\left(n_{a}=1\right)=\frac{N}{2}$ and $C_{i}\left(n_{a}=1\right)=0$, whereby

$$
C\left(n_{a}=1\right)=\frac{N}{2} \sigma^{0}
$$

and hence only the explicit $\sigma^{0}$ block survives.

\section{Nonabelian generalization}

For $n \geq 2$, the vacuum critical surface $\mathcal{C}_{(N, 1), \ldots,(N, 1)}$ is associated with the solution (3.9) which is a direct sum of $n$ irreducible $S U(2)$ representations of dimension $N$. This can clearly be obtained by repeating the above construction $n$ times. In particular, $V_{+}=\left(\Sigma_{+}\right)^{\oplus n}$ is a product of $n$ "marked cycles" as above. Notice, however, that the same saddle point is obtained if one acts with an additional permutation of the $2 n$ auxilliary radial coordinates $\bar{\lambda}_{i}=1, i=1, \ldots, 2 n$ (recall that the explicit entries $\pm \frac{N}{2}$ of $C$ are always isolated). In doing this, the decomposition of $V_{+}$into irreducible cycles gets modified. It can nonetheless be made into one irreducible cycle with $2 n$ 
marked points which come in groups of two at equal distance, for example. This demonstrates that the mapping between the Yang-Mills saddle-points and those of the abelianization approach in Section 7 is complicated. In particular, it is not injective. Again, this construction generalizes to blocks of the critical surfaces $\mathcal{C}_{\left(n_{1}, s_{1}\right), \ldots,\left(n_{k}, s_{k}\right)}$ corresponding to irreducible $S U(2)$ representations of various dimensionalities.

\section{Fluxons}

Fix an integer $1 \leq n \leq N$ and consider the block gauge field configuration of size $2 n$ given by

$$
\begin{aligned}
C & =\frac{N}{2} V\left(\sigma^{3} \otimes \cos (2 \rho)+\sigma^{2} \otimes \sin (2 \rho)\right) V^{-1} \\
& =\frac{N}{2}\left(\begin{array}{cc}
V_{+} \cos (2 \rho) V_{+}^{-1} & -\mathrm{i} V_{+} \sin (2 \rho) V_{-}^{-1} \\
\mathrm{i} V_{-} \sin (2 \rho) V_{+}^{-1} & -V_{-} \cos (2 \rho) V_{-}^{-1}
\end{array}\right),
\end{aligned}
$$

which is almost the same as (8.14) above but without the $\sigma^{0}$ block. We choose

$$
\lambda_{i}=-\frac{n-2 i}{n} \quad \text { for } \quad i=1, \ldots, n-1,
$$

along with

$$
V_{+}=\Sigma_{(n)} \quad \text { and } \quad V_{-}=\mathbb{1}_{n-1}
$$

where $\Sigma_{(n)} \in U(n+1)$ is a cyclic permutation matrix representing $\pi_{(n)}:=(12 \cdots n)$. Then we get explicitly

$$
C=\frac{N}{2 n}\left(\begin{array}{cc}
\operatorname{diag}\left(-n+\underset{\tilde{\xi}_{-}}{2, \ldots, n-2)}\right. & \tilde{\tilde{\xi}}_{+} \\
\operatorname{diag}(n-2, \ldots,-n+2)
\end{array}\right)
$$

where $\tilde{\xi}_{ \pm}$are cyclic operators (rather than raising/lowering operators as before).

In this case $C_{0}=0$, and hence this solution is part of the orbifold singularities for $n$ coincident fluxons in the moduli space (3.11) of Section 3.1, rather than an irreducible representation of the isometry group $S U(2)$. This construction is further used below. In particular, the special case $n=1$ gives a single fluxon $C(n=1)=\frac{N}{2} \sigma^{3}$. Then there exists a unitary transformation $U \in S U(2)$ such that

$$
U C(n=1) U^{-1}=\frac{N}{2} U \sigma^{3} U^{-1}=c_{i} \sigma^{i},
$$

which gives the position $c_{i}$ of the fluxon on the sphere $S^{2}$.

\section{Multi-block solutions}

Let us modify the previous radial solution by setting $\lambda_{1}= \pm 1$ and taking $\lambda_{i+1}$ to be given by (8.24), while keeping the angular variables $(8.25)$ in $U(n+2)$ and $U(n)$ the same. Then the block covariant coordinates $(8.23)$ of size $2(n+1)$ are given explicitly as

$$
C=\frac{N}{2 n}\left(\begin{array}{cc}
\operatorname{diag}(-n+2, \ldots, n-2, \pm n) & \xi_{+} \\
\xi_{-} & \operatorname{diag}(\mp n, n-2, \ldots,-n+2)
\end{array}\right),
$$

which is almost the same as the vacuum configuration (8.16) for an $n$-dimensional irreducible representation except that there are two explicit diagonal entries $\frac{N}{2},-\frac{N}{2}$ instead of $\frac{N}{2}, \frac{N}{2}$. In particular, $C_{0}$ is no longer constant and hence the gauge fields (8.28) are not solutions of the YangMills equations of motion. This can be cured by the addition of extra irreducible representations as follows. 
One can now construct solutions of the Yang-Mills equations with several blocks and arbitrary parameters, i.e. the generic critical surfaces $\mathcal{C}_{\left(n_{1}, s_{1}\right), \ldots,\left(n_{k}, s_{k}\right)}$, by joining an even number of copies of (8.28) in a suitable way. Fix another integer $m \geq 1$ such that $n+m \leq N$, and consider again the block covariant coordinate $(8.23)$ of size $2(n+m)$ with

$$
\lambda_{1}=1, \quad \lambda_{i}=-\frac{n-2(i-1)}{n} \quad \text { for } \quad i=2, \ldots, n \quad \text { and } \quad \lambda_{j+n-1}=-\frac{m-2(j-1)}{m} \quad \text { for } \quad j=1, \ldots, m .
$$

The angular degrees of freedom are given by

$$
V_{+}=\Sigma_{(n+m)} \quad \text { and } \quad V_{-}=\mathbb{1}_{n+m-1}
$$

in $U(n+m \pm 1)$, corresponding to the cyclic permutation $\pi_{(n+m)}$ decomposed as

$$
\pi_{(n+m)}=\left(\pi_{(n)}\right)_{1, \ldots, n} \circ\left(\pi_{(m)}\right)_{n+1, \ldots, n+m} \circ(1 n+1)
$$

where the subscripts indicate the indices that the permutations act on. The role of the transposition $(1 n+1)$ is to first interchange the explicit 1 and -1 in (8.29) for the upper block in (8.23), which then takes the form of two copies of the matrix (8.28) but with the correct explicit diagonal entries $\pm \frac{N}{2}$. Since $V_{+}=\Sigma_{(n+m)}$ corresponds to an irreducible cycle, $C$ is a direct sum of two irreducible representations with opposite sign and hence lives on the critical surface block $\mathcal{C}_{(n, 1),(m,-1)}$ with vanishing overall trace. This construction clearly generalizes to an arbitrary number of irreducible representations of the $S U(2)$ isometry group.

\subsection{Action of the gauge group}

Finally, let us describe how the gauge symmetry acts on the radially foliated solutions. Recall that the gauge group $G \cong S U(n N)$ is embedded in the symmetry group of the orbit space $\mathcal{O}$ as $\phi=\phi_{0} \otimes \sigma^{0}$ in the Lie algebra of $G \subset S U(2 n N)$. This embedding is well adapted to the modification of the radial coordinates in (8.12) by the permutation matrix $\Sigma_{0}$. Indeed, there is an embedding of the "diagonal" subgroup $U\left(n N_{-}\right) \subset U\left(n N_{+}\right) \times U\left(n N_{-}\right)$into $G$ given by taking $V_{-}$ into $\operatorname{diag}\left(\mathbb{1}_{n}, V_{-}\right) \otimes \sigma^{0}$ as

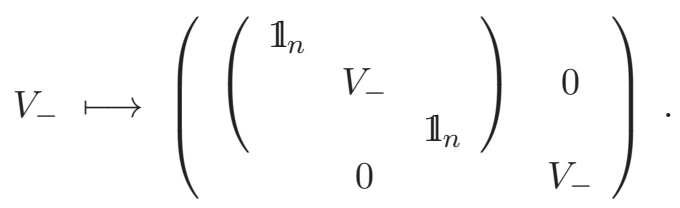

This shows explicitly that a large part of the gauge group is part of the stabilizer group $R=$ $U\left(n N_{+}\right) \times U\left(n N_{-}\right)$which defines the foliation of the radial coordinates.

Furthermore, there is an additional symmetry $S U(n) \subset U\left(n N_{+}\right)$embedded into $G$ by taking $U$ into $\operatorname{diag}\left(U, \mathbb{1}_{n N_{-}}\right) \otimes \sigma^{0}$ as

$$
U \longmapsto\left(\begin{array}{ccc}
U & & \\
& \mathbb{1}_{n N_{-}} & \\
& & U
\end{array}\right) \quad \begin{gathered}
0 \\
0
\end{gathered}
$$

This extra $S U(n)$ symmetry acts on the marked momenta $p_{1}, \ldots, p_{n}$ of Section 7.3 , and together with the degenerate Itzykson-Zuber localization it is thus responsible for the emergence of the nonabelian gauge symmetry in the commutative limit. The remainder $S U(n N) / S U\left(n N_{-}\right) \times S U(n)$ of the gauge group mixes the symplectic leaves, so that the radial foliation is not $G$-equivariant. 


\section{Acknowledgments}

We thank C.-S. Chu, B. Dolan, H. Grosse, X. Martin and D. O'Connor for helpful discussions. The work of H.S. was supported in part by the FWF Project P16779-N02 and in part by the FWF Project P18657. The work of R.J.S. was supported in part by the EU-RTN Network Grant MRTN-CT-2004-005104.

\section{References}

[1] J. Madore, "The Fuzzy Sphere", Class. Quant. Grav. 9 (1992) 69-88.

[2] H. Grosse, C. Klimcik and P. Presnajder, "Towards Finite Quantum Field Theory in Noncommutative Geometry", Int. J. Theor. Phys. 35 (1996) 231-244 [hep-th/9505175].

[3] C. Klimcik, "Gauge Theories on the Noncommutative Sphere", Commun. Math. Phys. 199 (1998) 257-279 [hep-th/9710153].

[4] U. Carow-Watamura and S. Watamura, "Noncommutative Geometry and Gauge Theory on Fuzzy Sphere", Commun. Math. Phys. 212 (2000) 395-413 [hep-th/9801195].

[5] S. Baez, A.P. Balachandran, B. Ydri and S. Vaidya, "Monopoles and Solitons in Fuzzy Physics", Commun. Math. Phys. 208 (2000) 787-798 [hep-th/9811169].

[6] H. Grosse, C.W. Rupp and A. Strohmaier, "Fuzzy Line Bundles, the Chern Character and Topological Charges over the Fuzzy Sphere", J. Geom. Phys. 42 (2002) 54-63 [math-ph/0105033].

[7] H. Grosse, M. Maceda, J. Madore and H. Steinacker, "Fuzzy Instantons", Int. J. Mod. Phys. A17 (2002) 2095 [hep-th/0107068].

[8] P. Presnajder, "Gauge Fields on the Fuzzy Sphere", Mod. Phys. Lett. A18 (2003) 2431-2438.

[9] H. Steinacker, "Quantized Gauge Theory on the Fuzzy Sphere as Random Matrix Model", Nucl. Phys. B679 (2004) 66-98 [hep-th/0307075].

[10] P. Castro-Villarreal, R. Delgadillo-Blando and B. Ydri, "A Gauge-Invariant UV-IR Mixing and the Corresponding Phase Transition for $U(1)$ Fields on the Fuzzy Sphere", Nucl. Phys. B704 (2005) 111-153 [hep-th/0405201].

[11] B. Ydri, " The One-Plaquette Model Limit of NC Gauge Theory in 2D", Nucl. Phys. B762 (2007) 148-188 [hep-th/0606206]; B. Ydri," Quantum Equivalence of NC and YM Gauge Theories in 2 D and Matrix Theory", [hep-th/0701057].

[12] P. Aschieri, T. Grammatikopoulos, H. Steinacker, G. Zoupanos, "Dynamical generation of fuzzy extra dimensions, dimensional reduction and symmetry breaking". JHEP 0609: 026, 2006. [hep-th/0606021].

[13] D. Karabali, V.P. Nair and A.P. Polychronakos, "Spectrum of Schrödinger Field in a Noncommutative Magnetic Monopole", Nucl. Phys. B627 (2002) 565-579 [ hep-th/0111249].

[14] A.Yu. Alekseev, A. Recknagel and V. Schomerus, "Noncommutative Worldvolume Geometries: Branes on $S U(2)$ and Fuzzy Spheres", J. High Energy Phys. 9909 (1999) 023 [hep-th/9908040]; "Brane Dynamics in Background Fluxes and Noncommutative Geometry", J. High Energy Phys. 0005 (2000) 010 [hep-th/0003187]. 
[15] S. Iso, Y. Kimura, K. Tanaka and K. Wakatsuki, "Noncommutative Gauge Theory on Fuzzy Sphere from Matrix Model", Nucl. Phys. B 604 (2001) 121-147 [hep-th/0101102].

[16] T. Azuma, S. Bal, K. Nagao and J. Nishimura, "Nonperturbative Studies of Fuzzy Spheres in a Matrix Model with the Chern-Simons Term", J. High Energy Phys. 0405 (2004) 005 [hep-th/0401038]; D. O'Connor, B. Ydri, " Monte Carlo Simulation of a NC Gauge Theory on The Fuzzy Sphere", JHEP 0611 (2006) 016, [hep-lat/0606013].

[17] D. Berenstein, J.M. Maldacena and H. Nastase, "Strings in Flat Space and pp-Waves from $\mathcal{N}=4$ Super Yang-Mills", J. High Energy Phys. 0204 (2002) 013 [hep-th/0202021].

[18] E. Witten, "Two-Dimensional Gauge Theories Revisited", J. Geom. Phys. 9 (1992) 303-368 [hep-th/9204083].

[19] J.A. Minahan and A.P. Polychronakos, "Classical Solutions for Two-Dimensional QCD on the Sphere", Nucl. Phys. B422 (1994) 172-194 [hep-th/9309119].

[20] D.J. Gross and A. Matytsin, "Instanton Induced Large $N$ Phase Transitions in Two-Dimensional and Four-Dimensional QCD", Nucl. Phys. B429 (1994) 50-74 [hep-th/9404004].

[21] M.R. Douglas and N.A. Nekrasov, "Noncommutative Field Theory", Rev. Mod. Phys. 73 (2001) 977-1029 [hep-th/0106048].

[22] R.J. Szabo, "Quantum Field Theory on Noncommutative Spaces", Phys. Rept. 378 (2003) 207-299 [hep-th/0109162].

[23] L.C. Jeffrey and F.C. Kirwan, "Localization for Nonabelian Group Actions", Topology 34 (1995) 291-327 [alg-geom/9307001]; "Intersection Theory on Moduli Spaces of Holomorphic Bundles of Arbitrary Rank on a Riemann Surface", Ann. Math. 148 (1998) 109-196 [alg-geom/9608029].

[24] P.-E. Paradan, "The Moment Map and Equivariant Cohomology with Generalized Coefficients", Topology 39 (2000) 401-444.

[25] L.C. Jeffrey, Y.-H. Kiem, F.C. Kirwan and J. Woolf, "Cohomology Pairings on Singular Quotients in Geometric Invariant Theory", Transf. Groups 8 (2003) 217-259 [math. AG/0101079].

[26] C.T. Woodward, "Localization for the Norm-Square of the Moment Map and the TwoDimensional Yang-Mills Integral", math.SG/0404413.

[27] C. Beasley and E. Witten, "Nonabelian Localization for Chern-Simons Theory", J. Diff. Geom. 70 (2005) 183-323 [hep-th/0503126].

[28] M. Blau and G. Thompson, "Localization and Diagonalization: A Review of Functional Integral Techniques for Low-Dimensional Gauge Theories and Topological Field Theories", J. Math. Phys. 36 (1995) 2192-2236 [hep-th/9501075].

[29] R.J. Szabo, "Equivariant Cohomology and Localization of Path Integrals", Lect. Notes Phys. M63 (2000) 1-319; "Equivariant Localization of Path Integrals", hep-th/9608068.

[30] A.A. Migdal, "Recursion Equations in Gauge Field Theories", Sov. Phys. JETP 42 (1975) 413 [Zh. Eksp. Teor. Fiz. 69 (1975) 810-822].

[31] B.E. Rusakov, "Loop Averages and Partition Functions in $U(N)$ Gauge Theory on TwoDimensional Manifolds", Mod. Phys. Lett. A5 (1990) 693-703. 
[32] L.D. Paniak and R.J. Szabo, "Instanton Expansion of Noncommutative Gauge Theory in Two Dimensions", Commun. Math. Phys. 243 (2003) 343-387 [hep-th/0203166].

[33] L.D. Paniak and R.J. Szabo, "Lectures on Two-Dimensional Noncommutative Gauge Theory 1: Classical Aspects", Sveske Fiz. Nauka A16 (2003) 1-27 [hep-th/0302195]; "Lectures on TwoDimensional Noncommutative Gauge Theory 2: Quantization", Lect. Notes Phys. 662 (2005) 205-237 [hep-th/0304268].

[34] L.D. Paniak and R.J. Szabo, "Open Wilson Lines and Group Theory of Noncommutative YangMills Theory in Two Dimensions", J. High Energy Phys. 0305 (2003) 029 [hep-th/0302162].

[35] D.J. Gross and N.A. Nekrasov, "Solitons in Noncommutative Gauge Theory", J. High Energy Phys. 0103 (2001) 044 [hep-th/0010090].

[36] S. Helgason, Differential Geometry, Lie Groups and Symmetric Spaces (Academic Press, New York, 1978); Groups and Geometric Analysis: Integral Geometry, Invariant Differential Operators and Spherical Functions (Academic Press, New York, 1984).

[37] M. Caselle and U. Magnea, "Random Matrix Theory and Symmetric Spaces", Phys. Rept. 394 (2004) 41-156 [cond-mat/0304363].

[38] R.J. Szabo, "Finite Volume Gauge Theory Partition Functions in Three Dimensions", Nucl. Phys. B723 (2005) 163-197 [hep-th/0504202].

[39] H. Grosse and H. Steinacker, "Finite Gauge Theory on Fuzzy $\mathbb{C} P^{2}$ ", Nucl. Phys. B707 (2005) 145-198 [hep-th/0407089].

[40] J. Madore, S. Schraml, P. Schupp and J. Wess, "Gauge Theory on Noncommutative Spaces", Eur. Phys. J. C 16 (2000) 161-167 [hep-th/0001203].

[41] N. Berline, E. Getzler and M. Vergne, Heat Kernels and Dirac Operators (Springer-Verlag, 1992).

[42] L. Griguolo, D. Seminara and R.J. Szabo, "Instantons, Fluxons and Open Gauge String Theory", Adv. Theor. Math. Phys. 9 (2005) 775-860 [hep-th/0411277].

[43] C.-S. Chu, J. Madore and H. Steinacker, "Scaling Limits of the Fuzzy Sphere at One Loop", J. High Energy Phys. 0108 (2001) 038 [hep-th/0106205].

[44] W. Behr, F. Meyer and H. Steinacker, "Gauge Theory on Fuzzy $S^{2} \times S^{2}$ and Regularization on Noncommutative $\mathbb{R}^{4}$, J. High Energy Phys. 0507 (2005) 040 [hep-th/0503041].

[45] V. Guillemin and S. Sternberg, Symplectic Techniques in Physics (Cambridge University Press, 1984).

[46] C. Itzykson and J.-B. Zuber, "The Planar Approximation. 2”, J. Math. Phys. 21 (1980) 411.

[47] V. Guillemin, E. Lerman and S. Sternberg, "On the Kostant Multiplicity Formula", J. Geom. Phys. 5 (1988) 721-750. 